University of New Hampshire

University of New Hampshire Scholars' Repository

$11-1-2013$

\title{
Helium, Oxygen, Proton, and Electron (HOPE) Mass Spectrometer for the Radiation Belt Storm Probes Mission
}

H. O. Funsten

R. M. Skoug

A. A. Guthrie

E. A. MacDonald

J. R. Baldonado

See next page for additional authors

Follow this and additional works at: https://scholars.unh.edu/physics_facpub

Part of the Physics Commons

\section{Recommended Citation}

Funsten, H. O.; Skoug, R. M.; Guthrie, A. A.; MacDonald, E. A.; Baldonado, J. R.; Harper, R. W.; Henderson, K. C.; Kihara, K. H.; Lake, J. E.; Larsen, B. A.; Puckett, A. D.; Vigil, V. J.; and Spence, Harlan E., "Helium, Oxygen, Proton, and Electron (HOPE) Mass Spectrometer for the Radiation Belt Storm Probes Mission" (2013). Space Science Reviews. 163.

https://scholars.unh.edu/physics_facpub/163

This Article is brought to you for free and open access by the Physics at University of New Hampshire Scholars' Repository. It has been accepted for inclusion in Physics Scholarship by an authorized administrator of University of New Hampshire Scholars' Repository. For more information, please contact Scholarly.Communication@unh.edu. 


\section{Authors}

H. O. Funsten, R. M. Skoug, A. A. Guthrie, E. A. MacDonald, J. R. Baldonado, R. W. Harper, K. C. Henderson, K. H. Kihara, J. E. Lake, B. A. Larsen, A. D. Puckett, V. J. Vigil, and Harlan E. Spence 


\title{
Helium, Oxygen, Proton, and Electron (HOPE) Mass Spectrometer for the Radiation Belt Storm Probes Mission
}

\author{
H.O. Funsten • R.M. Skoug • A.A. Guthrie · E.A. MacDonald • J.R. Baldonado • \\ R.W. Harper · K.C. Henderson · K.H. Kihara - J.E. Lake • B.A. Larsen · A.D. Puckett • \\ V.J. Vigil • R.H. Friedel • M.G. Henderson • J.T. Niehof • G.D. Reeves • M.F. Thomsen • \\ J.J. Hanley • D.E. George · J.-M. Jahn • S. Cortinas • A. De Los Santos • G. Dunn • \\ E. Edlund • M. Ferris • M. Freeman • M. Maple • C. Nunez $\cdot$ T. Taylor • W. Toczynski • \\ C. Urdiales $\cdot$ H.E. Spence $\cdot$ J.A. Cravens $\cdot$ L.L. Suther $\cdot$ J. Chen
}

Received: 13 September 2012 / Accepted: 12 February 2013 / Published online: 8 March 2013 (C) The Author(s) 2013. This article is published with open access at Springerlink.com

\begin{abstract}
The HOPE mass spectrometer of the Radiation Belt Storm Probes (RBSP) mission (renamed the Van Allen Probes) is designed to measure the in situ plasma ion and electron fluxes over $4 \pi \mathrm{sr}$ at each RBSP spacecraft within the terrestrial radiation belts. The scientific goal is to understand the underlying physical processes that govern the radiation belt structure and dynamics. Spectral measurements for both ions and electrons are acquired over $1 \mathrm{eV}$ to $50 \mathrm{keV}$ in $36 \mathrm{log}$-spaced steps at an energy resolution $\Delta E_{\mathrm{FWHM}} / E \approx 15 \%$. The dominant ion species $\left(\mathrm{H}^{+}, \mathrm{He}^{+}\right.$, and $\left.\mathrm{O}^{+}\right)$of the magnetosphere are identified using foil-based time-of-flight (TOF) mass spectrometry with channel electron multiplier (CEM) detectors. Angular measurements are derived using five polar pixels coplanar with the spacecraft spin axis, and up to 16 azimuthal bins are acquired for each polar pixel over time as the spacecraft spins. Ion and electron measurements are acquired on alternate spacecraft spins. HOPE incorporates several new methods to minimize and monitor the background induced by penetrating particles in the harsh environment of the radiation belts. The absolute efficiencies of detection are continuously monitored, enabling precise, quantitative measurements of electron and ion fluxes and ion species abundances throughout the mission. We describe
\end{abstract}

H.O. Funsten $(\varangle) \cdot$ R.M. Skoug · A.A. Guthrie · E.A. MacDonald · J.R. Baldonado · R.W. Harper ·

K.C. Henderson - K.H. Kihara - J.E. Lake · B.A. Larsen · A.D. Puckett · V.J. Vigil · R.H. Friedel ·

M.G. Henderson · J.T. Niehof · G.D. Reeves · M.F. Thomsen

Los Alamos National Laboratory, MS-D466, PO Box 1663, Los Alamos, NM 87545, USA

e-mail: hfunsten@lanl.gov

J.J. Hanley · D.E. George · J.-M. Jahn · S. Cortinas · A. De Los Santos · G. Dunn · E. Edlund · M. Ferris · M. Freeman · M. Maple · C. Nunez · T. Taylor · W. Toczynski · C. Urdiales

Southwest Research Institute, 6220 Culebra Road, San Antonio, TX 78238, USA

H.E. Spence $\cdot$ J.A. Cravens

University of New Hampshire, 39 College Road, Morse Hall, Durham, NH 03824, USA

L.L. Suther

Applied Physics Laboratory, Johns Hopkins University, Laurel, MD 20723, USA

J. Chen

Baja Technology, L.L.C., Tuscon, AZ 85721, USA 
the engineering approaches for plasma measurements in the radiation belts and present summaries of HOPE measurement strategy and performance.

Keywords Space plasma $\cdot$ Radiation belts $\cdot$ Ion mass spectrometry $\cdot$ Electron spectrometry · RBSP

\section{Introduction}

The Radiation Belt Storm Probes (RBSP) mission is designed to understand the influence of the Sun on the Earth's space environment by studying the structure and dynamics of the Earth's radiation belts and their response to energy and mass input by the solar wind. The primary scientific objectives of this mission include discovering the physical processes that govern acceleration and transport of radiation belt electrons and ions, understanding the systematic dynamic balance between particle acceleration and loss, and understanding the causal drivers of these processes in the context of geomagnetic storms. By studying and understanding these processes, we will better understand and predict space weather hazards and their impact on the world's space infrastructure as well as for the manned space program. The RBSP mission consists of two sun-pointing, spin stabilized spacecraft (A and B) with a nominal spin period $\tau_{\mathrm{SC}}$ of $\sim 12 \mathrm{sec}$. The spacecraft are placed into a $0.1 \times 4.8 \mathrm{R}_{\mathrm{E}}$ altitude orbit at a $10^{\circ}$ inclination.

The HOPE mass spectrometers, one for each of the two RBSP spacecraft and designated HOPE-A and HOPE-B, are one of three pairs of instruments of the Energetic Particle, Composition, and Thermal Plasma (ECT) suite (Spence et al., this issue), whose key science objectives in support of the RBSP mission include:

1. Determine the physical processes that produce radiation belt enhancement events,

2. Determine the dominant mechanisms for relativistic electron loss,

3. Determine how the inner magnetospheric plasma environment controls radiation belt acceleration and loss, and

4. Develop empirical and physical models for understanding and predicting radiation belt space weather effects

HOPE was designed to measure the crucial populations of the plasmasphere, plasma sheet, and lower-energy ring current, including the relative composition of the most abundant ion components $\left(\mathrm{H}^{+}, \mathrm{He}^{+}\right.$, and $\left.\mathrm{O}^{+}\right)$as well as electrons, over the $0.001-50 \mathrm{keV}$ energy range of the core plasma populations. The two other instruments on the ECT Suite, Relativistic Electron Proton Telescope (REPT) (Baker et al., this issue) and Magnetic Electron Ion Spectrometer (MagEIS) (Blake et al., this issue) extend the ion and electron flux measurements to $10 \mathrm{~s}$ of MeV. HOPE measurements will be used to understand how, when, and where various types of plasma waves are produced to provide quantitative understanding of their effects on radiation belt particles.

RBSP is the first mission dedicated to the comprehensive study of the physical processes that drive the structure and variability of the radiation belts. In situ measurement of this region of the Earth's space environment presents an extraordinary challenge due to the large fluxes of penetrating radiation. In particular, substantial electron fluxes are typically observed at energies that can exceed $15 \mathrm{MeV}$ in both the inner radiation belt (typically centered near $1.5 \mathrm{R}_{\mathrm{E}}$ ) and outer radiation belt (typically most intense near 4-5 $\mathrm{R}_{\mathrm{E}}$ at the Earth's magnetic equator), as well as inner belt proton energies that can exceed $100 \mathrm{MeV}$ (Selesnick et al. 2007). The penetrating radiation environment of the RBSP orbit is expected to produce 
substantial background counts in conventional plasma and ion mass spectrometers, which presents the primary challenge for the HOPE design and measurement strategy.

Because of the harsh penetrating radiation environment of the radiation belts, a primary driver in the design of HOPE was minimizing the background count rates in the electron multiplier detectors typically used for this type of measurement. Key mitigation strategies incorporated into the design include bulk and spot shielding, use of time-offlight measurements to reject uncorrelated events associated with penetrating radiation, careful charged particle optical design, and use of channel electron multiplier (CEM) detectors instead of microchannel plate detectors typically used for time-of-flight measurements.

The HOPE mass spectrometer measures the fluxes of $\mathrm{H}^{+}, \mathrm{He}^{+}, \mathrm{O}^{+}$and electrons over the energy range $0.001-50 \mathrm{keV}$. It has five angular pixels coplanar with the spacecraft spin axis, acquires a complete ion or electron measurement each spacecraft spin, and alternates between ion and electron measurements each spin. HOPE consists of four subsystems:

- The Door Subsystem consists of a once-open door that spans the entrance aperture of the instrument to protect the instrument from particulate contamination and to maintain a nitrogen gas purge before launch to prevent contamination from hydrocarbons and humidity to which the detectors are sensitive.

- The Electrostatic Energy Analyzer (ESA) Subsystem incorporates a "top-hat" ESA configuration that selects the charge of the incident plasma particle (ion or electron) as well as the energy-per-charge passband of the incident ion or electron.

- The Time-of-Flight (TOF) Subsystem measures the time $\tau_{\text {TOF }}$ elapsed when an ion or electron traverses a pathlength $\sim 3 \mathrm{~cm}$ between an ultrathin "Start" foil and a "Stop" anode. This measurement provides ion speed that, coupled with the energy measurement of the ESA Subsystem, provides ion mass.

- The Hope Electronics Unit (HEU) incorporates the electronics signal processing from the detectors including TOF, scalars, low voltage power supplies, high voltage power supplies, and communications with the spacecraft.

Because of the complexity of the HOPE measurement technique and strategy, we have developed a comprehensive end-to-end instrument performance model whose subsystem components have been individually and collectively validated through test and calibration. The next section describes the scientific objectives associated with HOPE and measurements needed to meet the scientific objectives. Detailed description of the HOPE mass spectrometer design, performance, and operation follow after this section.

\section{Scientific and Measurement Objectives}

Many other scientific missions have measured the electrons and/or ions that make up the bulk thermal and suprathermal plasma distributions in the inner magnetosphere. However, three aspects of the HOPE measurements make them unique. First, HOPE is the first mass spectrometer uniquely designed to minimize the effects of penetrating radiation that have severely impacted many other plasma measurements within the most intense portions of the radiation belts. Second, HOPE's measurements on both RBSP spacecraft will enable resolving plasma populations and physical processes in space and time over a large range of spatio-temporal separations. Third, HOPE is part of a comprehensive scientific payload that is, for the first time, capable of resolving the global-scale, local-scale, and meso-scale processes that control radiation belt processes and inner magnetosphere dynamics. 
The HOPE science requirements are driven directly by the RBSP mission requirements and, in turn, drive the instrument performance and measurement requirements. We divide the primary science topics that rely on the HOPE measurements into four areas: seed populations, sources of waves, substorms \& ring current, and plasmasphere.

\subsection{Seed Populations}

Unlike solar energetic ions, radiation belt electrons cannot penetrate the magnetosphere directly from the solar wind. Rather, the source of $\mathrm{MeV}$ electrons in the radiation belts is lower-energy electrons that obtain $\mathrm{MeV}$ energies through the action of one or more energization processes. Therefore the intensity (flux) of $\mathrm{MeV}$ electrons in the radiation belts is a product of the strength of the energization process(es) and the number of lower-energy electrons that are available to be energized. These lower-energy electrons are referred to as the "seed population" for radiation belt electrons (Baker et al. 1986; Obara et al. 2000).

However, there is no precise definition of the seed population for radiation belt electrons. Energization is continuous, e.g., $2 \mathrm{MeV}$ electrons were once $1 \mathrm{MeV}$ electrons, $1 \mathrm{MeV}$ electrons were once $500 \mathrm{keV}$ electrons, etc. Of particular interest is the relative importance of low energy electrons to the radiation belt seed populations, for which we must consider phase space densities and the adiabatic invariants defined by guiding center theory (Roederer 1967). The adiabatic (or Hamiltonian) invariants that are typically used in radiation belt physics are the gyro-invariant ( $\mu$ or $\mathrm{M}$ ) associated with gyration around the magnetic field, the bounce invariant ( $\mathrm{J}$ or $\mathrm{K}$ ) associated with motion along a field line between the mirror points, and the drift invariant ( $\Phi$, or $\mathrm{L}^{*}$ ) associated with gradient-curvature drift (Schulz and Lanzerotti 1974). Phase space density $f=j / p^{2}$ (where $j$ is the particle flux and $p$ is the momentum) is conserved along a particle's trajectory (in the absence of additions or losses). The adiabatic invariants are functions of the magnetic field which varies spatially and temporally. For example, the magnetic moment $\mu=p_{\perp}^{2} / B$ is conserved, so as a particle moves in a changing magnetic field, $B$, its momentum changes and the relationship between flux (as a function of position, energy and pitch angle) and phase space density (as a function of $\mu, \mathrm{K}$, and $\mathrm{L}^{*}$ ) changes. The HOPE instrument (and the entire ECT suite) was designed with the requirement for continuous measurements of phase space density, at a variety of $\mu$ and $\mathrm{K}$, over the entire RBSP orbit. The HOPE energy range was required to span from a few eV to $\sim 50 \mathrm{keV}$ with energy resolution $\Delta E_{\mathrm{FWHM}} / E<20 \%$ in order to provide contiguous energy coverage with good energy overlap with the MagEIS electron measurements (Blake et al., this issue) and ion measurements from the Radiation Belt Storm Probes Ion Composition Experiment (RBSPICE) instrument (Lanzerotti et al., this issue).

The conservation of adiabatic invariants implies that electrons that are transported over large radial distances can gain large amounts of energy. If we consider only radial transport processes, the energy range that constitutes the source population depends on where the electrons started. $\mathrm{MeV}$ electrons in the slot region $\left(\sim 2 \mathrm{R}_{\mathrm{E}}\right)$ will have a source population energy of $100 \mathrm{~s}$ of $\mathrm{keV}$ if they started at $5 \mathrm{R}_{\mathrm{E}}$ but if they were transported from $7 \mathrm{R}_{\mathrm{E}}$ (as shown for shock-associated events like the March 1991 CRRES event (Li et al. 1993)) the source population is electrons with energies of $10 \mathrm{~s}$ of $\mathrm{keV}$. Furthermore, it is not the radial transport per se that energizes the electrons, it is the change of magnetic field. Under extreme distortions of the geomagnetic field the radial gradients of field strength can also be extreme and radial transport can produce larger energization than under quiet conditions. The lowest possible energy from which seed populations can be accelerated to radiation belt energies during so-called "super storms" is not yet known. Understanding the dependence 
of $\mu$ on $E$ and $L$ over the bulk plasma population is a critical result expected from the HOPE measurements.

The above discussion describes the energy of seed populations that are directly energized by a single process such as betatron acceleration. An equally important question is the role of seed populations for $\mathrm{MeV}$ electrons that are accelerated through a combination of processes. Thermal plasma motion is controlled primarily by plasma $(E \times B)$ drift. However, energetic particle motion is controlled primarily by gradient-curvature drift. Intermediate energies undergo both types of motion. Therefore the energy-dependent phase space density profiles provide a powerful tool for analyzing and interpreting multi-step energization and the relationship between seed and radiation belt populations. For example, in one scenario, substorm injection or large scale convection transports electrons from the plasma sheet to the inner magnetosphere, energizing them from $100 \mathrm{~s}$ of $\mathrm{eV}$ to $10 \mathrm{~s}$ of $\mathrm{keV}$. Radial diffusion further transports and energizes the electrons to $100 \mathrm{~s}$ of $\mathrm{keV}$ near the plasmapause. Finally wave-particle interactions energize the electrons, locally, up to $\mathrm{MeV}$ energies. While this is just one, hypothetical, example, a predictive understanding of a radiation belt event relies on the spatial and energy distribution of seed electrons as much as on our ability to quantify the various energization processes.

\subsection{Sources of Waves}

There is a growing consensus that gyroresonant wave-particle interactions play a key role in the acceleration and loss of radiation belt electrons (Reeves et al. 2009) and, in particular, for equatorially-mirroring electrons (e.g. Taylor et al. 2004; Chen et al. 2007) as evidenced by peaks in the radial profiles of phase space density (e.g. Selesnick and Blake 1997; Green and Kivelson 2004). Gyroresonant wave-particle interactions are frequently referred to as a "local" acceleration (or loss) process because individual wave-particle interactions are temporally brief and spatially localized along a particular magnetic field line. Nevertheless, many of these interactions globally average over the drift motion of the electrons with a large net impact on the radiation belts. When local acceleration or loss occurs in combination with radial diffusion, additional mixing of processes occurs and is averaged over both the radial and azimuthal dimensions.

Generally, five different wave modes can interact strongly with radiation belt electrons (Horne and Thorne 1998) including magnetosonic (Horne et al. 2000) and Z-mode waves (Glauert and Horne 2005). Two of the most important wave populations are whistler mode chorus and electromagnetic ion cyclotron (EMIC) waves. Figure 1 (adapted from Summers et al. 1998; Reeves 2007) schematically shows the production of chorus and EMIC waves. Chorus waves are produced by the injection of plasma sheet electrons into the inner magnetosphere and their subsequent drift around the dawn side of the Earth. The injected electron populations exhibit temperature anisotropies (parallel and perpendicular to B) that are unstable to whistler-mode wave growth. Plasma sheet ions are also injected into the inner magnetosphere but, due to their opposite charge, drift around the dusk side of the magnetosphere where they can produce EMIC waves-particularly in high density regions such as plasmaspheric plumes. The nature of anisotropic energy and pitch angle distributions determines the nature of the waves that are produced which, in turn, determines the nature of interaction with relativistic electrons. Additionally, the characteristics of EMIC waves are strongly controlled by the relative abundances of plasma ions, predominantly $\mathrm{H}^{+}, \mathrm{He}^{+}$, and $\mathrm{O}^{+}$.

Plasma data can be used to test the instability conditions for various waves modes (Gary 1993). Instability thresholds for whistler (MacDonald et al. 2008), EMIC (Blum et al. 2009), 


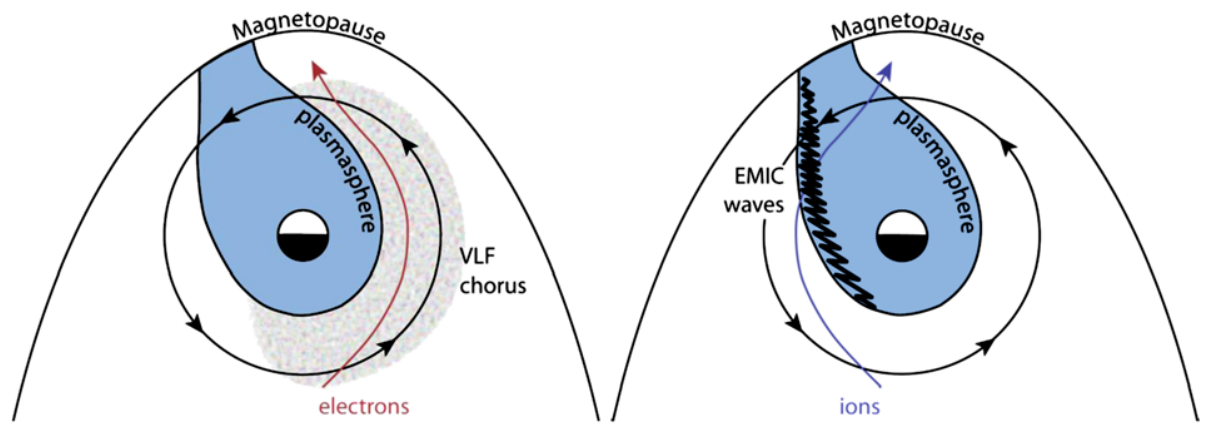

Plasmasheet Sources

Plasmasheet Sources

Fig. 1 A schematic showing the expected source of free energy for the generation of VLF chorus waves (left) and electromagnetic ion cyclotron waves (right) adapted from Reeves (2007). In this view of the equatorial plane, electrons injected from the plasma sheet drift Eastward, believed to be generated through the electron cyclotron instability by an anisotropic temperature distribution. This creates distributions that are unstable to the growth of chorus waves, while injected ions drift west where they can produce EMIC waves, particularly in high-density plasmaspheric plumes. These are only two possible generation mechanisms for a variety of waves that can interact with radiation belt electrons

and magnetosonic mode (Thomsen et al. 2011) waves have been studied using LANL MPA data from geosynchronous orbit. These techniques applied at geosynchronous orbit show good comparison with other measures of waves, either in situ, remote, or from ground-based measurements (e.g. Spasojevic et al. 2011; Blum et al. 2012). Superposed epoch analysis has been used to infer the relative intensity of growth and loss wave processes at geosynchronous orbit (MacDonald et al. 2010). RBSP on-board wave measurements will facilitate detailed comparison with the plasma distributions. In addition, for EMIC mode waves, measurements of the thermal ion composition will reduce assumptions in earlier analyses and enable more detailed discrimination between different bands of EMIC mode waves.

The HOPE mass spectrometer will measure the key plasma distributions (including energy/pitch angle distributions and composition ratios) needed to understand the creation of these waves, their properties (such as frequencies and wave normal), their propagation and damping, and spatial distribution. Detailed comparison of the plasma distributions and the wave properties measured by EMFISIS and EFW will provide critical tests of plasma physics theory including whether wave growth can be explained by quasilinear theory or whether nonlinear dynamics dominate the physics. These questions are particularly important in understanding how large-amplitude chorus (Cattell et al. 2008) is produced, whether it is just an extreme form of "regular" chorus, and what plasma conditions are responsible for such extremes.

The local plasma conditions that HOPE measures also control the propagation of waves away from their generation region, along (or across) magnetic field lines. Relativistic electrons can interact at any point on the field line, and the effects on the radiation belts can be quite different depending on where that interaction occurs. For example, chorus waves are generated at the magnetic equator. Their wave normal angle controls the direction of propagation, but the suprathermal plasma distributions control the Landau damping of the waves and therefore how far off the equator they will propagate (Bortnik et al. 2007) as illustrated in Fig. 2 (from Reeves et al. 2009). Near the equator, wave-particle interactions affect electrons with equatorial pitch angles near $90^{\circ}$ where energization dominates. At higher 


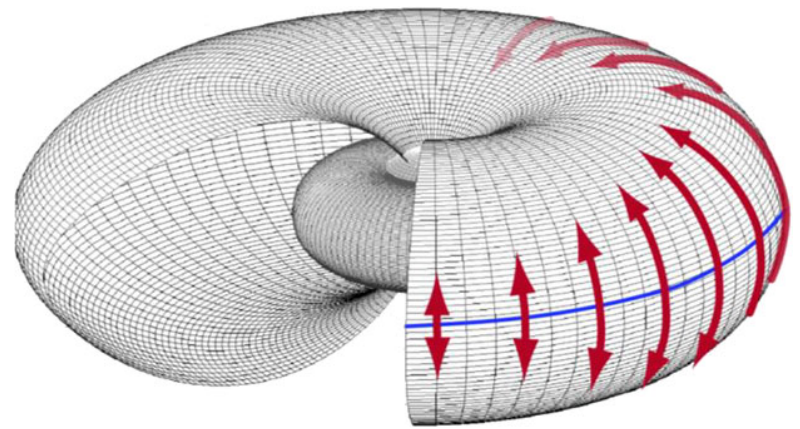

Fig. 2 Chorus waves propagate from the equator following a specific pattern. Electrons that are injected on the nightside drift eastward toward noon. Near midnight (in the gap in the outer torus), chorus waves are strongly damped and stay near the equator. As the electrons drift, their distributions change, allowing chorus waves to propagate to higher latitudes, where they may also further intensify before they eventually lose their energy (see Bortnik et al. 2007). From Reeves et al. (2009)

latitudes, more field-aligned electrons are more strongly affected, and, for those equatorial pitch angles, energization is less rapid but pitch angle scattering is stronger. Therefore whether chorus produces net enhancement or loss of radiation belt electrons may be strongly dependent on the latitudinal extent of the chorus waves as well as the presence and amount of damping.

As discussed above, the net effect on the radiation belts from "local" acceleration and pitch angle diffusion derives from the drift-averaged effect of all local interactions. To date, all theoretical predictions of "global" wave particle interactions take local energy and pitch angle diffusion coefficients and drift-average this ensemble by applying a uniform weighting. A more accurate drift averaging would account for the magnetic local time (MLT), radial distance (since drift paths are not at constant radius) and even temporal dependence of the diffusion coefficients, i.e., $\langle D\rangle=\int D(M L T, R, t)$.

The two-spacecraft configuration of the RBSP mission, assisted by global modeling, is critical for establishing the spatial, temporal, and activity-dependent correlations that enable extrapolation of local measurements to the global scale. Testing competing theories and mechanisms requires measurements that can constrain each step in the complex chain of coupled global plasma dynamics associated with wave generation, propagation, and coupling to radiation belt electrons. Our ability to quantify these steps will ultimately shape our ability to predict, for example, whether a storm will increase or decrease radiation belt fluxes (Reeves et al. 2003).

\subsection{Substorms \& Ring Current}

Geomagnetic storms and substorms have a profound effect on the configuration and dynamics of the inner magnetosphere including the radiation belts. Storms and radiation belt events occur together so often that they are sometimes considered to be synonymous. Figure 3, from Reeves (1998), illustrates the strong association between storm activity and geosynchronous $\mathrm{MeV}$ electron fluxes. However, that same study as well as subsequent studies showed that this relationship is complex and that stronger storms do not necessarily have a stronger effect on the radiation belts. Nearly all the processes that are thought to strongly affect the radiation belts (radial transport, wave-generation, electron energization, pitch angle scattering, magnetopause losses, etc.) are generally stronger during storms compared to quiet times. 


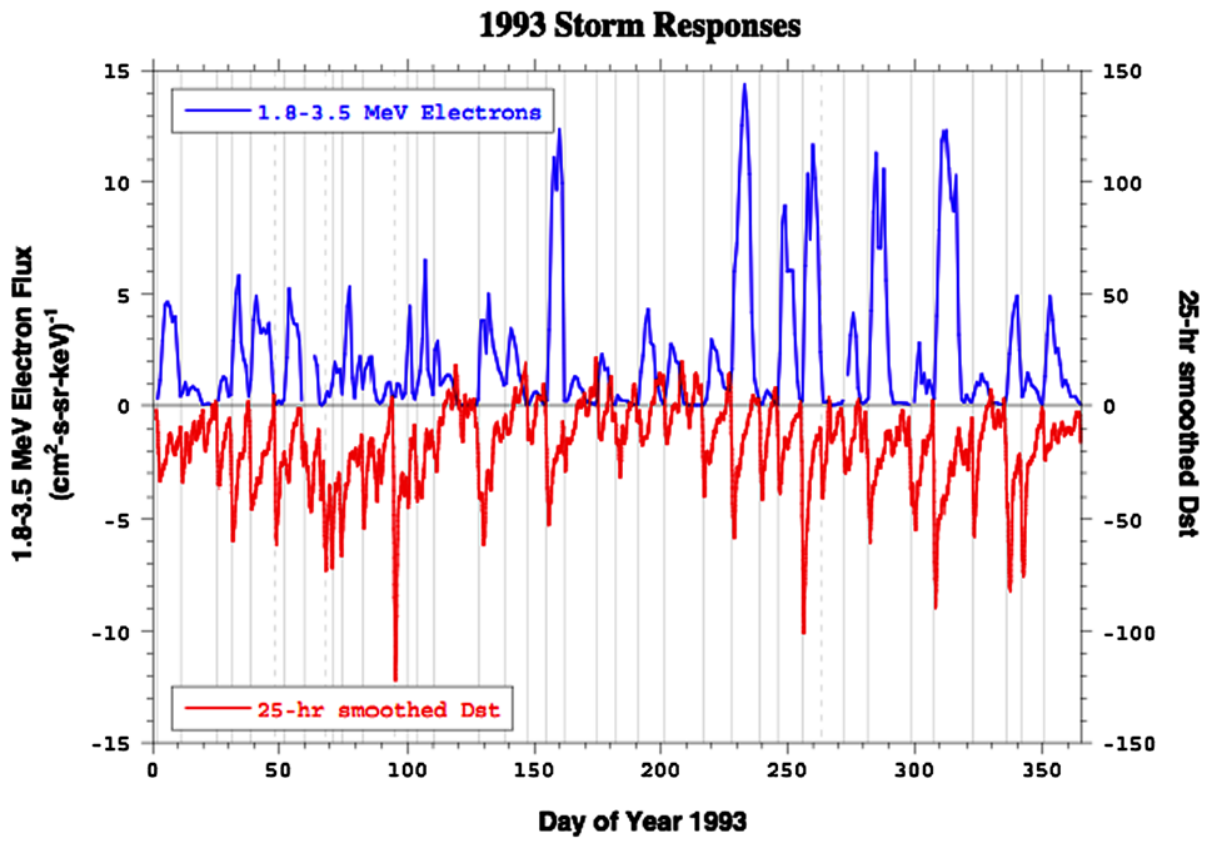

Fig. 3 Geosynchronous relativistic electron fluxes (blue) and smoothed Dst (red) for the year 1993 show a clear association between storm activity and electron flux enhancements. However, it is apparent that there is not a one-to-one occurrence and that the intensities of electron fluxes and Dst minima are poorly correlated. The precise relationship between radiation belt electron events and geomagnetic storms remains a mystery

Fundamental open questions that will be addressed by the RBSP measurements include the relationship between storms and the radiation belts, whether a storm is a necessary condition for a radiation belt event, and whether and how we can predict the effects of a given storm.

The HOPE and RBSPICE instruments are highly-complementary components of the RBSP mission that will provide continuous and complete measurements of the ring current from essentially 0 to approximately $400 \mathrm{keV}$. They will both provide information about the ion composition of the ring current that has been a critical, missing input for ring current models (Daglis et al. 1999). The strength of a magnetic storm, as measured by Dst, is proportional to the total energy in the ring current (Dessler and Parker 1959; Sckopke 1966), which is, in turn, a function of the energy density spectrum and the composition. Figure 4 shows an example from the RAM ring current model (Jordanova et al. 1994) for a single point in the ring current with composition ratios that are independent of energy. Observations from HOPE and RBSPICE on the two RBSP satellites will allow us to determine the spatial, temporal, and activity-dependent correlations across the ring current and relate changes in those distributions to the global electric and magnetic fields.

Ring current physics, a critical RBSP science objective, is also profoundly important for interpreting radiation belt observations. As discussed above, the adiabatic invariants of radiation belt particle motion are essential parameters for understanding radiation belt processes. The first invariant, $\mu$, is a function of the local field which can be measured directly, but the second and third invariants, $\mathrm{K}$ and $\mathrm{L}^{*}$, are integrals along a field line and around a drift shell, respectively, and are dependent on a quantitative knowledge of the global magnetic field that can only be obtained from models. 
Fig. 4 The differential energy density of ring current ions predicted by the RAM model for an arbitrary geomagnetic storm (courtesy, V. Jordanova). Ions in the HOPE energy range $(<\sim 50 \mathrm{keV})$ are the source of the high-energy ring current but also comprise a substantial portion of the total energy density in the ring current

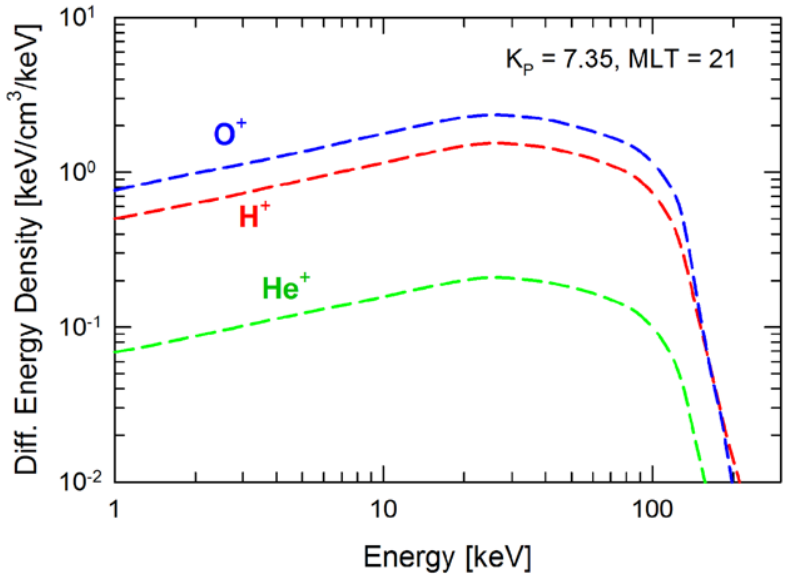

Figure 5 shows the profound effect of storm-time magnetic field reconfigurations on relativistic electron phase space density calculations-particularly calculation of the third invariant $\mathrm{L}^{*}$ (Reeves et al. 2012). As the ring current builds up diamagnetic currents, the geomagnetic field "inflates". In order to conserve the third invariant (the total magnetic flux enclosed in a drift shell), particles move outward, simultaneously changing energy and pitch angle to conserve $\mu$ and $\mathrm{K}$. This adiabatic rearrangement of particles is often called the "Dst effect" (Kim and Chan 1997). Figure 5 shows the Dst and smoothed Dst indices (top) and phase space density at fixed $\mu$ and $\mathrm{K}$ (bottom) for three geosynchronous spacecraft. While the geosynchronous satellites orbit at fixed radius, the drift shells move across their fixed positions which is equivalent to a "motion" in the $\mathrm{L}^{*}$ coordinate. Because of these and other factors, the HOPE ring current measurements are intimately linked to studies of phase space density gradients for relativistic electrons and for independently assessing adiabatic and non-adiabatic effects.

Storm-time dynamics cannot be fully studied without including the processes associated with substorms, but non-storm-time (isolated) substorms can also have important effects on the inner magnetosphere and the radiation belts. It has been hypothesized that substorms may directly inject radiation belt electrons (Ingraham et al. 2001), but this mechanism has not yet been fully verified. It is also possible that individual injections can produce the same unstable plasma distributions and wave populations that are produced in storms but with different characteristics (spatial distribution, energies, etc.) and understanding the continuum of activity from isolated substorms to major storms may be key to understanding the balance of competing processes. In addition to the contributions discussed previously, HOPE measurements play a key role in understanding substorms by characterizing the plasma sheet, by measuring the penetration depth and azimuthal extend of substorm injected populations, by tracing the energy-dependent drifts and drift boundaries of electrons and ions, and by measuring the interaction of plasmas with different ionospheric and magnetospheric origins.

\subsection{Plasmasphere}

The RBSP mission will also provide a unique, new set of observations of the plasmasphere. While plasmaspheric physics is not a direct objective of the RBSP mission, it plays a key supporting role in the primary RBSP science objectives and is a rich target for collateral scientific discovery. 
Fig. 5 The phase space density (PSD) (color coded) as a function of $\mathrm{L}^{*}$ for three geosynchronous satellites during a geomagnetic storm in 2001. As the ring current intensifies the drift shells "inflate" which produces an "apparent motion" of the satellites to very low $\mathrm{L}^{*}$. From Reeves et al. (2012)

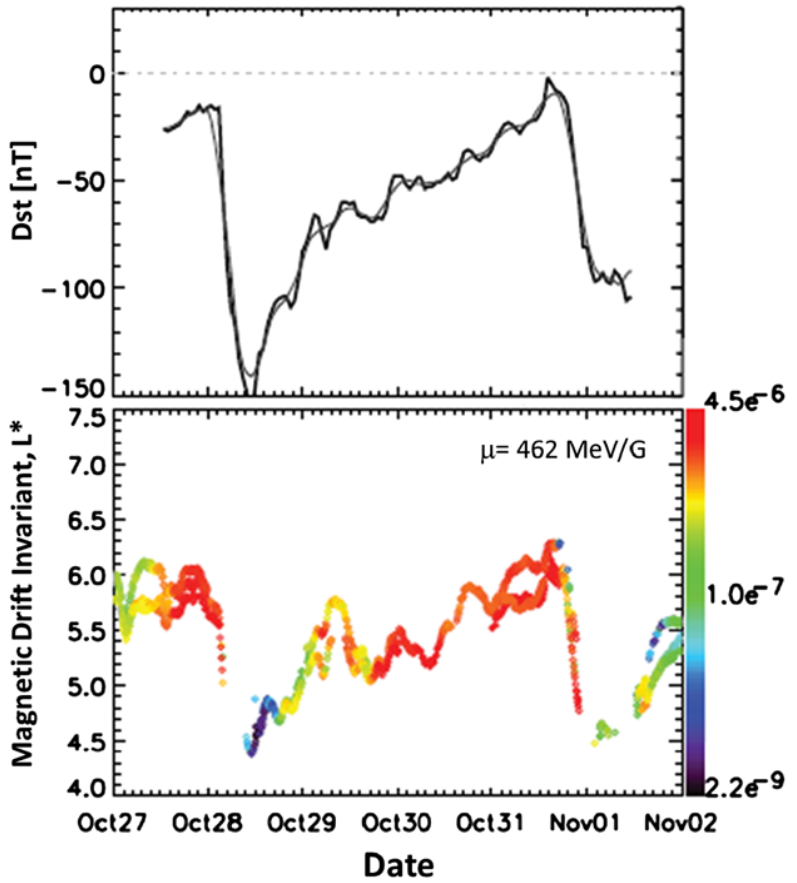

Many of the unique aspects of HOPE measurements of plasmaspheric populations come from the two-satellite configuration of RBSP. As the satellites lap one another in their orbits, we will obtain radial profiles of the plasmasphere separated in time by minutes to hours. At the low L-shells of the plasmapause, the inbound and outbound legs of the orbit are separated in MLT by large angles. The plasmapause and structures within it respond to changes in the large-scale convective electric field on a variety of time scales that have yet to be fully investigated (Carpenter 1995).

The dynamics of the plasmasphere also have a direct effect on the structure and dynamics of the relativistic radiation belt electrons. Lyons and Thorne (1973) showed that the equilibrium structure of the electron radiation belts-the inner belt, slot region, and outer belt — could be explained by a balance between radial diffusion rates (as a function of L-shell) and losses due to atmospheric precipitation from whistler hiss inside the plasmasphere. Li et al. (2001) used SAMPEX data to produce a dramatic illustration correlating the inner edge of the outer electron belt with the location of the plasmapause. Figure 6, adapted from their paper, shows MeV electron fluxes from 1992 to 2004 as a function of "McIlwain" L. Also plotted are sunspot number, solar wind velocity, and the plasmapause location based on a statistical model (Carpenter and Anderson 1992). Both the SAMPEX electron flux and plasmapause location were averaged over 30 days to highlight losses consistent with average loss lifetimes in the plasmasphere of roughly 10 days.

Statistical models such as Carpenter and Anderson (1992) or O'Brien and Moldwin (2003) treat the equatorial plasmasphere statistically, considering only large scale features, an inadequate approximation for radiation belt physics applications. As with chorus or EMIC waves, the effects of pitch angle scattering by plasmaspheric hiss must be averaged over the drift path of an electron. For drift paths fully within the plasmasphere, simple models of day-night asymmetry may suffice, but, for drift paths that intersect the plasmasphere 


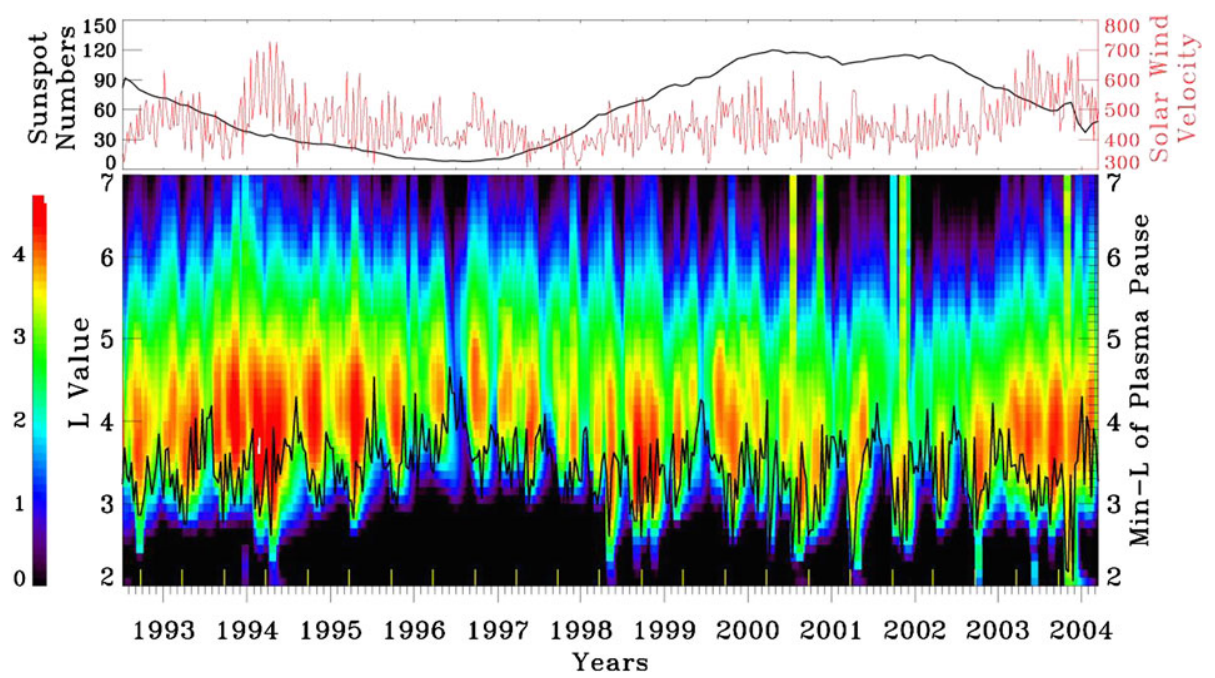

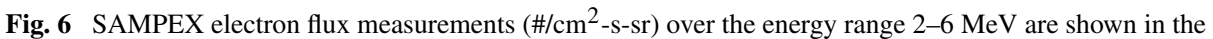
bottom panel. The yellow vertical bars on the horizontal axis are marks of equinoxes. The color bar is log scale, and L values are in bins of $0.1 \mathrm{~L}$. Sunspot number and average solar wind velocity for the same period are shown in the top panel. Adapted from Li et al. (2001)

over only part of the orbit, the shape of the plasmasphere is critical. Plasmaspheric plumes drain the outer layers of the plasmasphere to the magnetopause and, when they are present, they intersect all trapped electron orbits (see Fig. 1).

As previously discussed, plasmaspheric plumes play another role in the production, propagation, and effects of EMIC waves. Generally the ring current lies outside the location of the plasmasphere, although they can overlap because the low energy plasmaspheric plasma and higher energy ring current plasma follow different trajectories. Since storms produce both stronger ring currents and plasmaspheric plumes, this overlap typically occurs during storms. This is shown schematically in Fig. 7 from the cover of the AGU monograph on Inner Magnetosphere Physics and Modeling (Pulkkinen et al. 2005) (courtesy J. Goldstein). The figure shows the plasmasphere and drainage plume derived using EUV images and the modeled ring current from ENA observations from the IMAGE mission. This interaction is thought to produce EMIC waves which have been proposed as the cause of the dropout of relativistic electrons seen during the main phase of storms (e.g. Summers et al. 1998). However, questions remain as to whether the interaction always produces EMIC waves and, if so, whether those waves cause radiation belt dropouts (e.g. Meredith et al. 2003; Turner et al. 2012).

\subsection{Summary of HOPE Science Objectives}

The HOPE measurement requirements traceability starts with the RBSP mission's science objectives. Achieving the mission science objectives depends on answering key science questions such as in Table 1. For example, a key science question needed to determine the physical processes that produce radiation belt enhancement events is knowing where, when, and how local acceleration leads to relativistic electron acceleration. For example: How are 
Fig. 7 This figure illustrates the overlap between plasmaspheric drainage plumes (in green, derived from IMAGE EUV observations) and energetic ring current ions (in orange, derived from inversion of ENA observations)

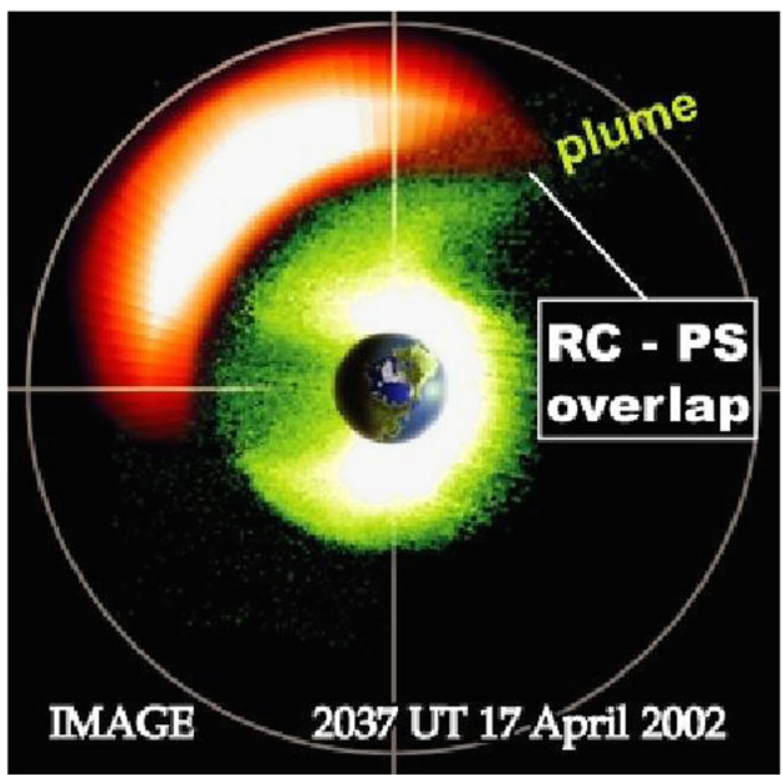

plasma waves produced? What are the plasma distributions that provide the free energy? How do those waves interact with relativistic electrons? What are the effects on energy and pitch angle distributions? These questions lead to specific science measurement objectives. Those that are specific to the ECT suite include measuring radial phase space distributions and gradients, measuring energy and pitch angle distributions for both plasma and radiation belt particles, and measuring the plasma density and composition. Those measurements enable us to test the cause and effect of specific energization processes. The measurement objectives for the ECT suite drive specific measurement requirements for each instrument in the suite as shown in the final column of Table 1 . The implementation of these scientific objectives through the HOPE mass spectrometer is the focus of his paper.

\section{HOPE Mass Spectrometer Description}

Because of the intensity of the penetrating particle flux in the radiation belts, the overall design and operation philosophy of HOPE was driven toward minimizing the effects of this penetrating radiation on the measurement of plasma ions and electrons. We have incorporated six primary strategies to address this issue. First, substantial resources (including modeling, simulation, and validation as well as instrument mass) were invested in shielding. The interior of the instrument was protected by a minimum wall thickness of $0.89 \mathrm{~cm}$ Aluminum and nearly $1.8 \mathrm{~cm}$ in the vicinity of the channel electron multiplier (CEM) detectors. Because of the exponential dependence of attenuation of energetic particles on shielding thickness, mating surfaces were stepped at their interfaces, and machined features such as vent holes were strategically placed to prevent a low-shielding pathway for energetic particles to penetrate into the instrument. Second, we exploit the time-of-flight measurement, in which a correlated pair of events from a single incident plasma ion or electron is measured, to reject uncorrelated detected events associated with penetrating radiation. Third, we incorporate CEM detectors instead of microchannel plate (MCP) detectors, which are 
Table 1 Key scientific and measurement goals for the HOPE mass spectrometer

\begin{tabular}{llll}
\hline Science objectives & Key science questions & $\begin{array}{l}\text { Science measurement } \\
\text { objectives }\end{array}$ & $\begin{array}{l}\text { HOPE } \\
\text { measurement } \\
\text { requirements }\end{array}$ \\
\hline $\begin{array}{l}\text { Determine the physical } \\
\text { processes that produce } \\
\text { radiation belt } \\
\text { enhancement events }\end{array}$ & $\begin{array}{l}\text { What processes are } \\
\text { responsible for radial } \\
\text { transport and } \\
\text { acceleration? }\end{array}$ & $\begin{array}{l}\text { Radial phase space } \\
\text { density (PSD) } \\
\text { distribution }\end{array}$ & $\begin{array}{l}\text { Electron energy } \\
(20-50 \mathrm{keV}) \text { and } \\
\text { pitch angle }\end{array}$ \\
& $\begin{array}{l}\text { Where, when, and how } \\
\text { do local acceleration } \\
\text { processes produce } \\
\text { radiation belt } \\
\text { acceleration? }\end{array}$ & $\begin{array}{l}\text { Radial PSD distribution, } \\
\text { local PSD gradients, } \\
\text { pitch angle distribution, } \\
\text { plasma density and } \\
\text { composition }\end{array}$ & $\begin{array}{l}\text { Electron energy } \\
(10-50 \text { keV) and } \\
\text { pitch angle; } \\
\text { plasma density } \\
\text { and composition } \\
\left(\mathrm{H}^{+}, \text {He } \mathrm{O}^{+} \text {, }\right.\end{array}$ \\
& $\begin{array}{l}\text { How does “prompt } \\
\text { acceleration" create new } \\
\text { radiation belts? }\end{array}$ & $\begin{array}{l}\text { Radial PSD distribution, } \\
\text { ultrarelativistic } \\
\text { population, energetic }\end{array}$ & $\begin{array}{l}\text { Electron energy } \\
\left(30-50 \mathrm{keV}^{+} \text {and }\right. \\
\text { pitch angle }\end{array}$
\end{tabular}

particle composition

How do plasma 'seed' or Radial PSD distribution source populations influence the characteristics of relativistic electron events?

Determine the dominant mechanisms for relativistic electron loss

Determine how the inner magnetospheric plasma environment controls radiation belt transport, acceleration, and loss

Develop empirical and physical models for understanding and predicting radiation belt space weather effects
Where, when, and how are radiation belt electrons scattered into the atmospheric loss cone?

What is the contribution of magnetopause shadowing to relativistic electron loss?

How do we characterize/model adiabatic transport of radiation belt particles?

How do the global and local characteristics of the plasma sheet and plasmaspheric populations control radiation belt structure, acceleration, and loss?

How do we predict and model spatial, spectral, and temporal characteristics of the radiation belt enhancements, over both short and long times?
Full electron pitch-angle distribution and intensities through outer zone regions

Full electron pitch-angle distribution and intensities through outer zone regions

Pitch angle distribution of ions and electrons; energy density of ring current

Effects of ring current and plasmapause structure on the outer radiation belt

Radial PSD distribution over wide range of solar wind and geophysical input conditions
Electron energy (30-50 keV) and pitch angle

Ions and electrons, $1 \mathrm{eV}-50 \mathrm{keV}$; composition $\left(\mathrm{H}^{+}\right.$, $\mathrm{He}^{+}, \mathrm{O}^{+}$)

Ions and electrons, $1 \mathrm{eV}-50 \mathrm{keV}$; composition $\left(\mathrm{H}^{+}\right.$, $\mathrm{He}^{+}, \mathrm{O}^{+}$)

Ions and electrons, $1 \mathrm{eV}-50 \mathrm{keV}$

Ion and electron energy (1 eV-50 keV) and pitch angle 
typically used for plasma time-of-flight mass spectrometry. We have found that CEMs are $\sim 20$ times less sensitive to penetrating radiation per $\mathrm{cm}^{2}$ of detection area. Independent pairs of CEM detectors are used for each of the five polar angle pixels; the two CEMs of a pair are separated by $1.96 \mathrm{~cm}$ center-to-center to minimize the probability that a single penetrating particle traverses and stimulates counts in both detectors, resulting in a coincidence. This is the first use of CEM detectors for time-of-flight measurement of plasma ions in space. Fourth, the charged particle optics design was carefully constructed so that only secondary electrons generated at locations relevant to the TOF measurement (specifically, the foil backside and the Stop anode) are steered to and collected by the appropriate CEM detector; secondary electrons generated at other surfaces inside the instrument by penetrating radiation cannot reach a CEM and therefore cannot be detected. Fifth, we continuously monitor and report both the count rate of correlated TOF measurements and rates of uncorrelated Start and Stop events. This provides a direct measure of the level of penetrating radiation. Furthermore, during times of low penetrating particle flux, we can calculate from these rates the absolute detection efficiency of the TOF subsystem, enabling quantitative monitoring of the instrument health and performance for the duration of the mission. And sixth, we additionally monitor penetrating radiation by reporting counts registered in long TOF bins in electron mode, which primarily result from coincidences from uncorrelated penetrating radiation, as well as short TOF coincidences in ion mode that are much faster than $\mathrm{H}^{+}$and result from individual penetrating particles.

\subsection{Overview}

The HOPE mass spectrometer is located on the $+\hat{u}$ panel of each RBSP spacecraft as shown in Fig. 8. The HOPE field-of-view (FOV) is fan-shaped, with polar angle $\theta$ viewing that extends nearly $180^{\circ}$, from $+\hat{w}$ (the direction of the Sun) to $-\hat{w}$. The center of the HOPE FOV views normal to the panel on which HOPE is attached, such that $\theta=0^{\circ}$ corresponds to $+\hat{u}$. Because the HOPE FOV lies in the same plane as the RBSP spin axis, HOPE therefore uses the spin of the spacecraft to sample nearly $4 \pi$ of space. The panel on which HOPE is placed lies between the panels on which solar panels are attached so that the solar panels do not obstruct the HOPE FOV.

The HOPE mass spectrometer, illustrated in Fig. 9, consists of three sensor subsystems as well as the Hope Electronics Unit (HEU) subsystem. We describe the HOPE spectrometer by following a charged particle through each sensor subsystem. The particle first transits the door subsystem, which consists of a once-open door that spans the entrance aperture and is retracted during instrument commissioning. The particle then enters the toroidal "top-hat" electrostatic energy analyzer (ESA) subsystem, which has a fan-shaped FOV (nearly $180^{\circ}$ in polar angle $\theta$ and $4.5^{\circ} \mathrm{FWHM}$ in azimuthal angle $\phi$ ) coplanar with the spacecraft spin axis. The ESA subsystem accepts only charged particles within a specific energy-per-charge $(E / q)$ range as defined by the bipolar, adjustable bias $V_{\mathrm{ESA}}$ applied to its inner toroidal dome. Because we expect the preponderance of ions to be singly charged $(q=+1)$, we henceforth assume that all ions detected are singly ionized and that the ESA subsystem provides measurement of an ion's energy $E$. $V_{\mathrm{ESA}}$ is defined using an adjustable bipolar power supply with maximum voltage $\pm 7.5 \mathrm{kV}$ that toggles polarity every $12 \mathrm{~s}$ (the nominal RBSP spin period) so that ions and electrons are measured during alternate spins.

A charged particle exiting the ESA subsystem enters the TOF subsystem, which has five independent polar pixels, each incorporating a linear, foil-based time-of-flight measurement. The pixels are arrayed at polar angles $\theta$ corresponding to $0^{\circ}$ (perpendicular to the spin axis), $\pm 36^{\circ}$, and $\pm 72^{\circ}$. The TOF drift boxes for the pixels are together biased to $V_{\mathrm{TOF}}=-11 \mathrm{kV}$ in 


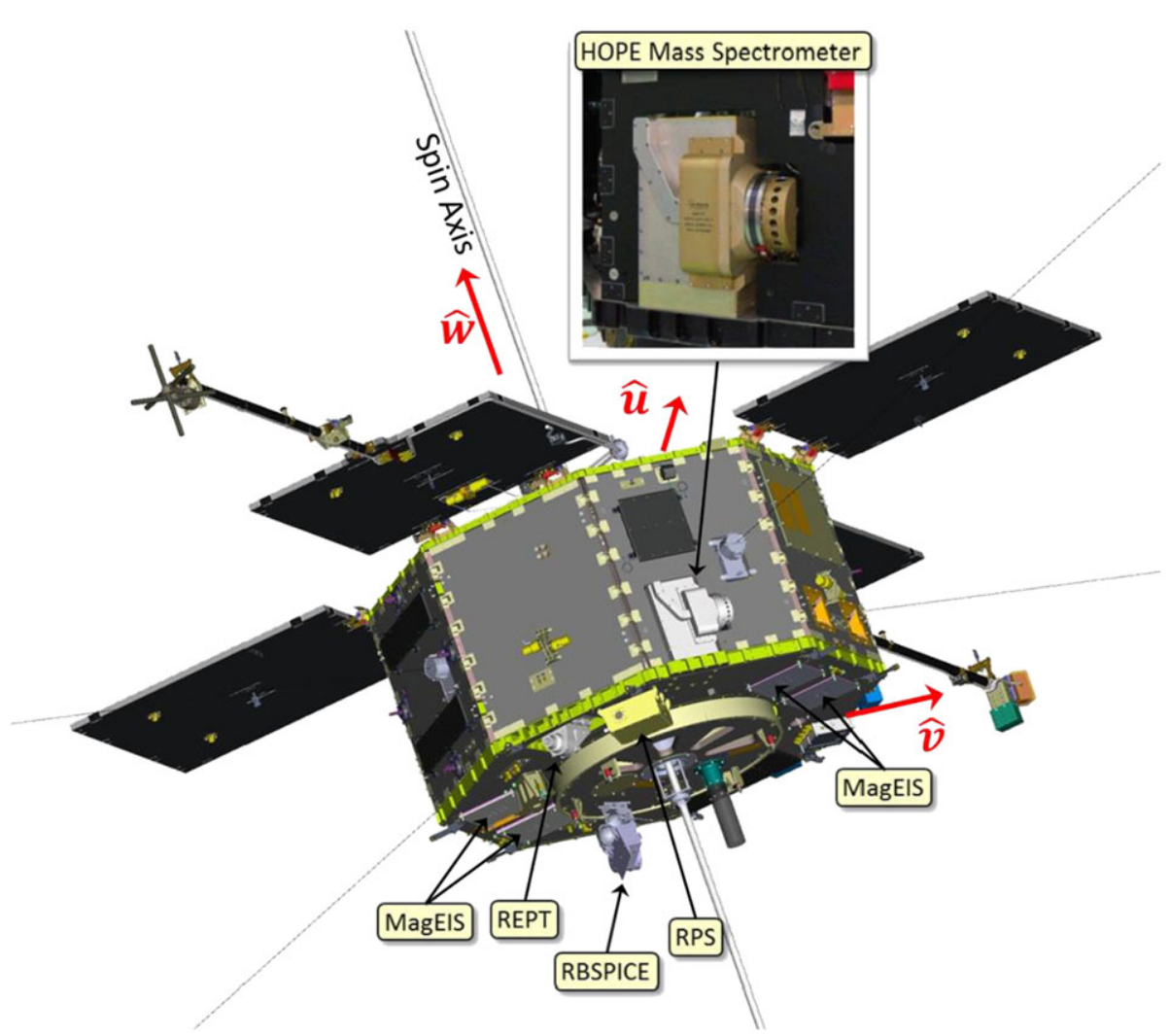

Fig. 8 HOPE is accommodated on the $+\hat{u}$ panel of the RBSP spacecraft, and the center of HOPE's fieldof-view is $+\hat{u}$. The sun lies in the $+\hat{w}$ direction. The inset photo shows HOPE-A integrated onto the spacecraft, adjacent to the EFW wire boom antenna. The other particle instruments on RBSP, shown in the figure, are the Magnetic Electron Ion Spectrometer (MagEIS), Relativistic Electron Proton Telescope (REPT), Relativistic Proton Spectrometer (RPS), and Radiation Belt Storm Probes Ion Composition Experiment (RBSPICE)

ion mode and $V_{\mathrm{TOF}}=+1.5 \mathrm{kV}$ in electron mode to accelerate the particles into the foil. This acceleration helps mitigate the energy straggling and angular scattering effects of the foil. This bias scheme also enables constant front and rear CEM detector biases, independent of whether ions or electrons are being measured. In the drift box, a charged particle transits the foil and generates secondary electrons from the foil's exit surface that are detected and start a timer. The charged particle then traverses the drift region of length $\sim 3 \mathrm{~cm}$, whereupon it strikes the Stop anode, generating Stop secondary electrons that are detected and stop the timer. Particle speed is derived from the ratio of the travel distance over the elapsed time. Particle mass is then derived from speed measured in the TOF subsystem and energy measured in the ESA subsystem. A summary of HOPE performance and resources are listed in Table 2.

The instrument response to a charged particle is a function of its species as well as its incident energy $E$, polar angle $(\theta)$ and azimuth angle $(\phi)$. Our approach to design, characterize and quantify instrument performance has been to first develop performance models for each subsystem and validate these subsystem performance models by testing of prototype and flight subsystems. We then couple these models into an end-to-end performance 


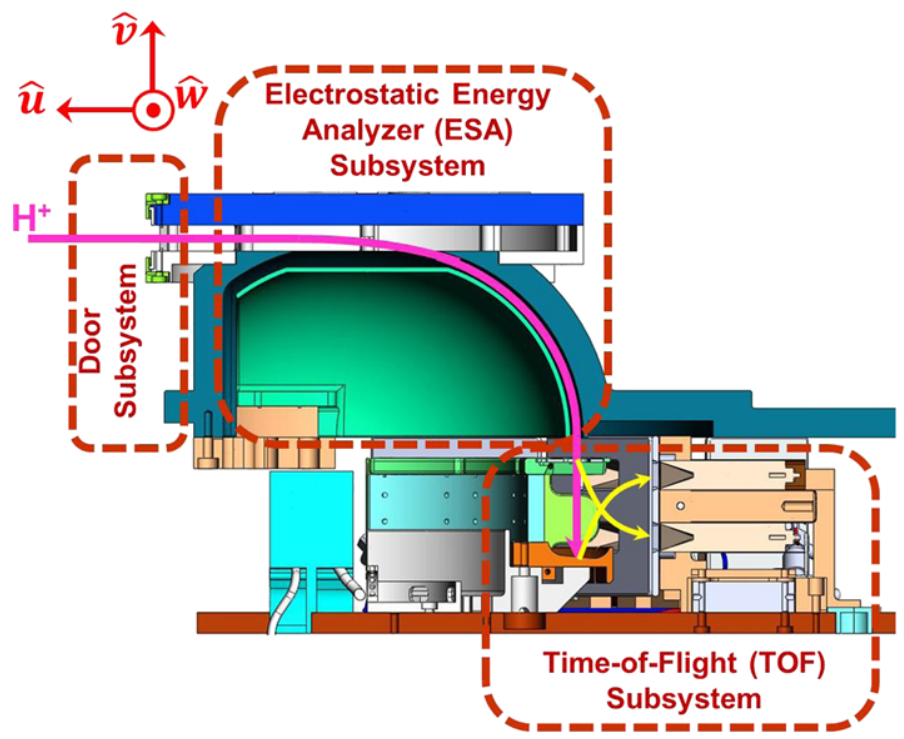

Fig. 9 Cross section of the HOPE mass spectrometer illustrating the three sensor subsystems and the trajectory of a proton through the instrument (magenta) and the trajectories of secondary electrons generated by the impact of the proton with the start foil and stop anode (yellow). The HOPE Electronics Unit (HEU) subsystem is not shown

model that is validated through flight instrument calibration. This section describes the sensor subsystems as well as the validation testing of the subsystems and their models.

\subsection{Sensor Subsystems}

The design of the HOPE sensor subsystems was driven by the challenging measurement environment of the radiation belts. We therefore also include a section on radiation analysis of the instrument.

\subsubsection{Door Subsystem}

The once-open door, successfully opened as one of the first activities of instrument commissioning, provides a barrier to particulate contamination that can cause electrostatic discharge points in regions of high electric fields (such as in the ESA subsystem). It additionally serves as a non-hermetic seal for the $\mathrm{N}_{2}$ purge of the instrument interior that helps prevent migration of volatile hydrocarbons and water to the CEM detectors, whose performance could be degraded by significant exposure to these species. The door was closed during transport of the flight units, during most spacecraft-level testing, and through launch; the instrument was purged with $\mathrm{N}_{2}$ up to launch.

Figure 10 schematically shows the door in the closed and opened configurations. The door, made of 0.10 -mm-thick 718 Inconel, is self-retracting with a force of $1.6 \mathrm{~N}$ around a hub that is located outside of the aperture FOV. The top and bottom edges of the door are captured within channel guides located above and below the entrance aperture of the ESA subsystem. Key surfaces of the guides and door were coated with Dicronite to minimize friction and prevent generation of particulate during testing. The action of the door is similar 
Table 2 Summary of HOPE Mass Spectrometer performance and resources

\begin{tabular}{ll}
\hline Species & $\mathrm{H}^{+}, \mathrm{He}^{+}, \mathrm{O}^{+}, \mathrm{e}^{-}$ \\
Energy range & $0.001-50 \mathrm{keV}$ \\
Energy resolution & $16 \% @ 1 \mathrm{eV}, 12 \% @ 50 \mathrm{keV}$ \\
Energy steps (reported) & 36 \\
Energy sweep period & $750 \mathrm{~ms}$ \\
Mass resolution, $m / \Delta m$ & $\geq 2 \mathrm{FWHM}$ \\
Polar pixels, center locations $(\theta)$ & $0^{0}, \pm 36^{\circ}, \pm 72^{\circ}$ \\
Polar angle $(\theta)$ resolution & $18^{\circ} \mathrm{Full} \mathrm{width}$ \\
Azim. angle $(\phi)$ resolution & $4.5^{\circ} \mathrm{FWHM}$ \\
Azim. sector angle $\left(\phi_{\mathrm{S}}\right)$ width & $750 \mathrm{~ms} / \tau_{\mathrm{SC}} \times 360^{\circ}$ \\
& $\left(22.5^{\circ} \mathrm{for} \tau_{\mathrm{SC}}=12 \mathrm{sec}\right)$ \\
Dimensions & $19.6 \mathrm{~cm} \times 19.6 \mathrm{~cm} \times 33.5 \mathrm{~cm}$ \\
Mass: HOPE A, HOPE B & $18.06 \mathrm{~kg}, 18.07 \mathrm{~kg}$ \\
Power (BOL, predicted EOL) & $18.2 \mathrm{~W}, 23.0 \mathrm{~W}$ \\
Telemetry Rate & $9.32 \mathrm{kbps}$ \\
$\mathrm{H}^{+}$Geometric Factor per polar pixel, $G_{\mathrm{I}}\left(\mathrm{cm}^{2} \mathrm{sreV} / \mathrm{eV}\right)$ & $1.25 \times 10^{-3} \mathrm{~cm}^{2} \mathrm{sr} @ 1 \mathrm{eV}$ \\
& $1.3 \times 10^{-3} \mathrm{~cm}^{2} \mathrm{sr} @ 50 \mathrm{keV}$ \\
Electron Geometric Factor per polar pixel, $G_{\mathrm{E}}\left(\mathrm{cm}{ }^{2} \mathrm{sr} \mathrm{eV} / \mathrm{eV}\right)$ & $2 \times 10^{-4} \mathrm{~cm}^{2} \mathrm{sr} @ 1 \mathrm{eV}$ \\
& $1.1 \times 10^{-5} \mathrm{~cm}^{2} \mathrm{sr} @ 50 \mathrm{keV}$ \\
Temperature limits (operating, survival) & $-25 \mathrm{to}+55^{\circ} \mathrm{C},-30 \mathrm{to}+60{ }^{\circ} \mathrm{C}$ \\
\hline
\end{tabular}

to that of a self-retracting tape measure. The end of the door has a multipurpose tang: it holds the door in closed position, it prevents full retraction of the door around the hub, and it is used as a handle to close the door when it is manually reset.

In its closed position, the door is kept in place by an "L"-shaped pivot arm, with one end connected to a TiNi shaped memory alloy (SMA) pin-puller and a tab at the other end contacting the door tang. The pivot arm uses a constant force $(10 \mathrm{lb} / \mathrm{in})$ coil spring. Actuation, performed by command to the instrument, retracts the pin of the pin-puller, allowing the pivot arm to rotate and release the tang. This releases the door, which self-retracts over the entire HOPE entrance aperture. The door and pin-puller must be manually reset after activation. Prototype and engineering models of the door were fabricated and extensively tested to validate the design. The engineering model was deployed in excess of 100 times without a single failure.

\subsubsection{Electrostatic Energy Analysis (ESA) Subsystem}

The ESA subsystem consists of a toroidal top-hat electrostatic analyzer (ESA) (Carlson et al. 1983; Young et al. 1988) that allows particles of a specific charge (+ or - ) and within a specific energy-per-charge $(E / q)$ range and angular range to pass to the TOF subsystem, where they are detected. For our time-of-flight classification scheme, we assume that the ions measured by HOPE are singly ionized, i.e., $q= \pm 1$. The top-hat geometry efficiently rejects ultraviolet light, which can stimulate the CEM detectors, from the instrument interior. The ESAs are blackened by the Ebonol-C process to further enhance UV rejection.

The top hat consists of a parallel plate entrance section that accepts charged particles over a polar angle $\theta$ range of nearly $180^{\circ}$ and acts as a collimator and restricts the azimuthal $(\phi)$ field-of-view. The angular field-of-view is thus fan-shaped $\left(180^{\circ} \times 4.5^{\circ}\right)$, with the center of the fan at $(\theta, \phi)=\left(0^{\circ}, 0^{\circ}\right)$ aligned in the $+\hat{u}$ spacecraft direction and perpendicular to the 
Fig. 10 Door (blue) and pivot arm (yellow) in closed (top panel) and open (bottom panel) configurations. The mechanism that releases the pivot arm is a shaped memory alloy pinpuller (orange cylinder, above door)
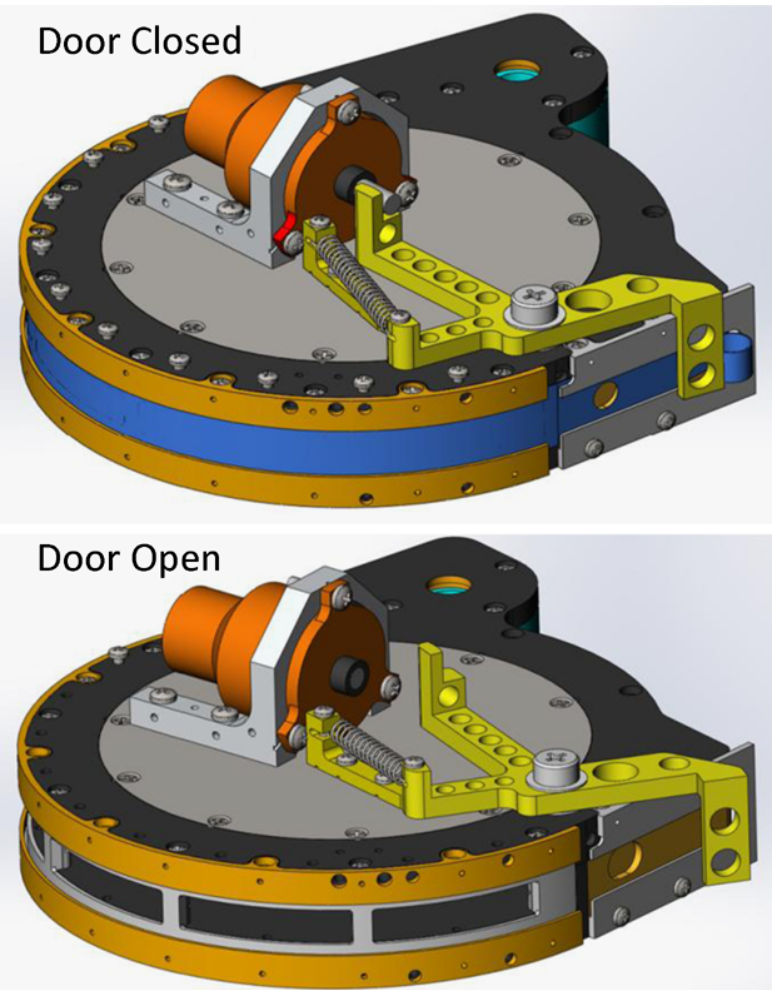

spin axis. As the spacecraft spins, HOPE samples a complete $2 \pi$ radians in azimuth over each spacecraft spin, forming the fundamental cadence for accumulation of a complete ion or electron histogram (shown in Fig. 38).

A charged particle that transits the top hat entrance section next encounters concentric electrodes in a toroidal geometry with a bending angle of $90^{\circ}$. The outer electrode, which lies at ground potential and serves as an exterior wall for the instrument, has bending radius of $59 \mathrm{~mm}$. The inner electrode, biased to $V_{\mathrm{ESA}}$, has a $7 \mathrm{~mm}$ toroidal radius and a central bending radius $55 \mathrm{~mm}$, resulting in a plate separation of $4.0 \mathrm{~mm}$. $V_{\mathrm{ESA}}$ is set using an adjustable bipolar power supply with maximum voltage $\left|V_{\mathrm{ESA}}\right|=7.5 \mathrm{kV}$; the polarity toggles between spacecraft spins, allowing measurement of ions and electrons for negative and positive polarity, respectively.

The HOPE top hat charged particle optics design was modeled using SIMION (Dahl 2000 ) in $2.5 \mathrm{D}$ that exploited the cylindrical symmetry of the top-hat. In general, the optics of electrostatic fields scale linearly with applied voltage, are independent of charged particle mass, and are charge-symmetric (particles of opposite charge follow identical trajectories if electrode polarities are reversed). However, this model also includes the closest components of the TOF subsystem (the ultrathin foil and its mounting plate at the entrance of the TOF drift box, all biased to $\left.V_{\mathrm{TOF}}\right)$ to properly couple the performance models of the ESA and TOF subsystems. Because $V_{\text {TOF }}$ is different between ion $\left(V_{\text {TOF }}=-11.0 \mathrm{kV}\right)$ and electron $\left(V_{\mathrm{TOF}}=+1.5 \mathrm{kV}\right)$ detection modes and remains constant as $V_{\mathrm{ESA}}$ changes, we both expect and observe small variations in the energy, angle, and throughput response of the ESA subsystem to ions and electrons. The ESA and TOF subsystem models are coupled at the planar entrance surface of the foil, and the ion and electron spatial and velocity distributions at 
Fig. 11 The geometric factor $G_{\text {ESA }}$ per polar pixel for the ESA subsystem varies systematically with energy as $G_{\mathrm{P}} \sim \log (E)$ and exhibits a small difference between incident ions (black symbols) and electrons (blue symbols). The lines are empirical fits

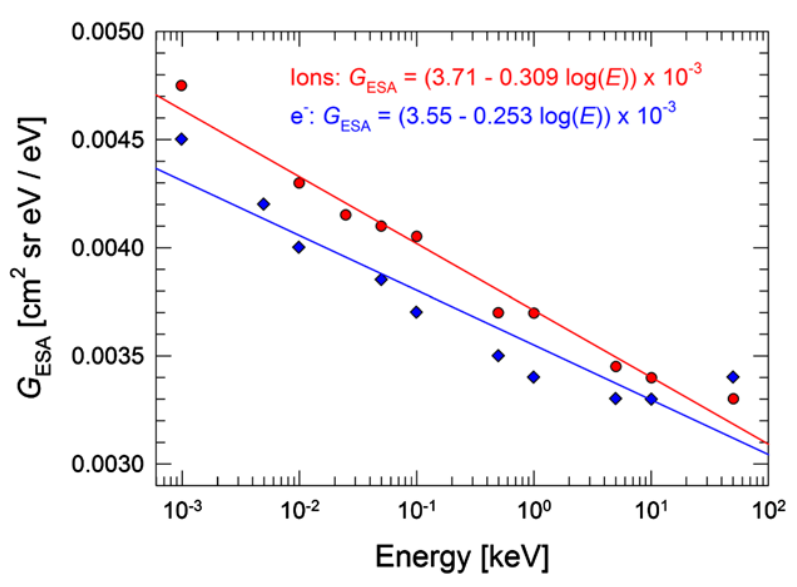

this location derived from the ESA subsystem performance model are input into the TOF subsystem performance model.

SIMION simulations of the ESA were performed for both protons and electrons over the full HOPE energy range of $1 \mathrm{eV}-50 \mathrm{keV}$ under two types of incident angle conditions. First, simulations were run with a parallel beam of input particles, corresponding to fixed incident polar and azimuthal directions, which best describes the laboratory ion and electron beams for validation through test and calibration (discussed below in connection with HOPE calibration measurements). Second, simulations were run with incident ions and electrons uniformly distributed over all angles and energies within the acceptance range of the instrument, allowing determination of the instrument geometric factors for an ambient charged particle flux that uniformly fills a pixel in angle and energy, which is more representative of the magnetospheric environment.

These simulations derived the full width at half maximum (FWHM) energy resolution $\Delta E_{\mathrm{FWHM}} / E \approx 15 \%$ and the azimuth angle resolution $\Delta \phi$ of $\sim 10^{\circ}$ full width (FW) and $\sim 5^{\circ} \mathrm{FWHM}$. The geometric factor for a toroidal electrostatic is derived using Gosling et al. (1978) and Young et al. (1988). Figure 11 shows the resulting geometric factor $G_{\mathrm{ESA}}$ for the ESA subsystem per polar pixel (which each span $18^{\circ}$ in polar angle) as a function of incident energy for both protons and electrons. Because particles of the same charge and energy but different mass follow identical trajectories in a static electric field, the proton results apply identically to $\mathrm{He}^{+}$and $\mathrm{O}^{+} . G_{\mathrm{ESA}}$ is found to depend slightly on energy as $G_{\mathrm{ESA}} \sim \log (E)$, with small differences between ions and electrons. These simulations were used to appropriately size the foil.

The ESA analyzer constant $k=E_{\mathrm{C}} / V_{\mathrm{ESA}}$ is a key parameter describing the central energy $E_{\mathrm{C}}$ of an energy passband as a function of the applied bias $V_{\mathrm{ESA}}$. The analyzer constant is based on the ESA geometry but can vary slightly due to factors such as error in the concentricity of the electrodes and fabrication variations within the machining tolerances of the electrodes. The value $k=7.035$ was derived for both HOPE-A and HOPE-B during calibration, in good agreement with the value of $k=6.90$ derived using the charged particle optics model for an ideal instrument with no alignment or machining imperfections.

As the spacecraft spins through $360^{\circ}$ in azimuthal angle, HOPE systematically acquires ion or electron energy histograms over five swaths of polar angle. Furthermore, $V_{\mathrm{ESA}}$ is stepped over the full instrument energy range $(1 \mathrm{eV}-50 \mathrm{keV}) 16$ times every 12 seconds, which is the nominal spacecraft spin period. Therefore, HOPE acquires a complete energy spectrum at 80 locations over the full sky; we selectively combine some azimuthal pixels 

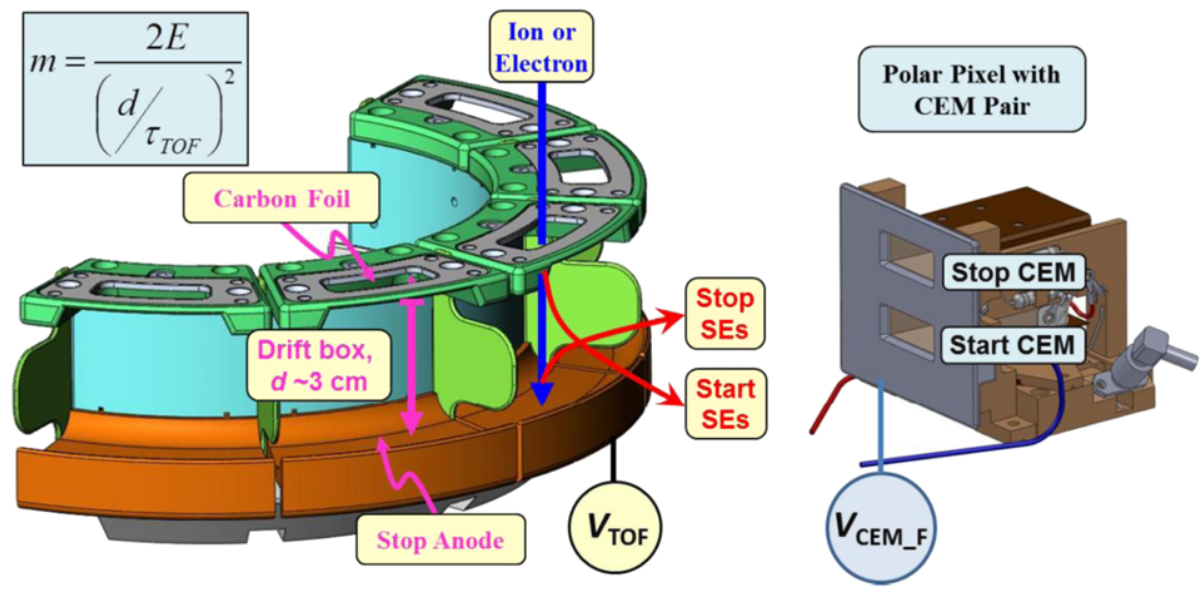

Fig. 12 The TOF subsystem consists of 5 independent polar pixels, each with a TOF drift box and pair of CEM detectors. Ion speed is determined by its time-of-flight $\tau_{\text {TOF }}$ over $\sim 3 \mathrm{~cm}$ between the carbon foil and stop anode

with overlapping fields-of-view at higher polar angles and reduce these to 40 locations that are nearly uniformly distributed over $4 \pi$ sr from which we derive the full plasma distribution function. Details of this strategy are provided later in Sect. 4.2.1.

\subsubsection{Time-of-Flight Subsystem}

The TOF Subsystem was designed to allow robust species separation of $\mathrm{H}^{+}, \mathrm{He}^{+}$, and $\mathrm{O}^{+}$ as well as a coincidence measurement of incident electrons within a high penetrating background environment. HOPE employs a standard linear, foil-based TOF measurement technique (e.g., Wüest 1998). Because this is a standard technique as a diagnostic for space plasma ions, the physical processes governing almost all aspects of its performance have been individually studied and documented.

A charged particle exiting the ESA subsystem enters the TOF subsystem (Fig. 12), which consists of five independent polar pixels arrayed at polar angles of $0^{\circ}$ (perpendicular to the spin axis), $\pm 36^{\circ}$, and $\pm 72^{\circ}$. Each polar pixel has a TOF drift box biased to $V_{\text {TOF }}$ and consists of an ultrathin carbon foil at the entrance, a drift region of $\sim 3 \mathrm{~cm}$, and a Stop Anode, which is shaped like a trough. The charged particle transits the foil, generating secondary electrons at the foil's exit surface that are steered to the "Start" CEM detector by the tailored electric field between the TOF drift box and the CEM detectors. Detection of a Start pulse initiates a timer. The particle continues to the Stop Anode, where it generates "Stop" secondary electrons that are optically steered to the Stop CEM detector, generating a Stop pulse that stops the timer. The charged particle's speed is calculated from the measurement of its time-of-flight $\tau_{\text {TOF }}$ over the drift region. We can calculate the charged particle's mass from knowledge of its speed in the TOF subsystem and its incident energy $E$ from the ESA subsystem. In practice, for each $V_{\mathrm{ESA}}$ setting (i.e., each energy passband), the times-of-flight of $\mathrm{H}^{+}, \mathrm{He}^{+}$, and $\mathrm{O}^{+}$uniquely lie within separate TOF ranges; instead of calculating the timeof-flight of individual ions as they are detected, we identify the ion's species at each energy by binning each TOF measurement within these unique TOF windows.

The full TOF window is $160 \mathrm{~ns}$, which is divided into 64 time bins, each of which is $2.5 \mathrm{~ns}$. This window is longer than required, as the slowest ions (incident $1 \mathrm{eV} \mathrm{O}^{+}$accelerated by $V_{\mathrm{TOF}}=-11 \mathrm{kV}$ ) have a characteristic TOF of $\sim 100 \mathrm{~ns}$; in contrast, the fastest 
Fig. 13 The HOPE TOF

Subsystem consists of five polar pixels each with a pair (Start and Stop) of CEM detectors. Polar pixels are identified from 1 to 5 in a clockwise direction as shown in the top photograph. Polar Pixel 3 is centered on the $+\hat{u}$ spacecraft vector (refer to Figs. 8 and 9). Polar Pixels 1 and 5 lie in the $\hat{u}-\hat{v}$ plane, with Pixel 1 located $18^{\circ}$ from $-\hat{v}$ (anti-sunward) and Pixel 5 located $18^{\circ}$ from $+\hat{v}$ (sunward). A close-up of HOPE-B Pixels 2-4 is shown in the lower photograph. The CEMs are mounted behind a plate electrode that resides at the same bias as the front of each CEM pair, $V_{\text {CEM_F }}$

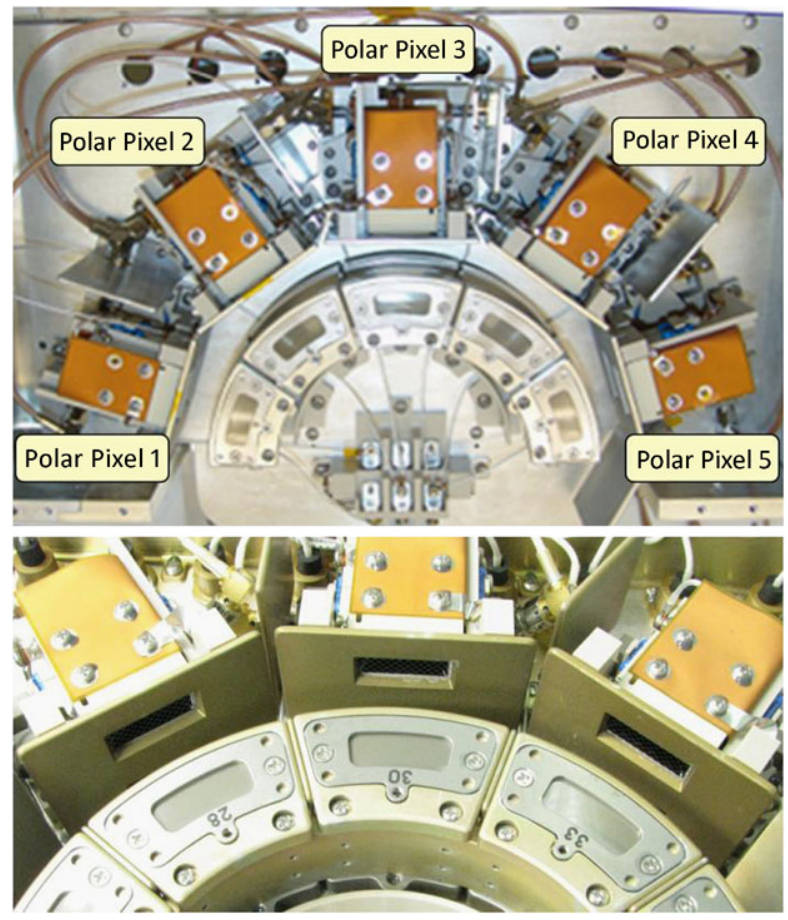

ion TOF measurement is $3-4 \mathrm{~ns}$ for $50 \mathrm{keV} \mathrm{H}^{+}$. Because the TOF of incident $1 \mathrm{eV}-50 \mathrm{keV}$ electrons is short $\left(\tau_{\mathrm{TOF}}<2 \mathrm{~ns}\right.$ ), in electron mode we use a short TOF measurement only as a coincidence measurement for electrons and retain longer TOF events to monitor background penetrating radiation. We have incorporated a $\sim 10 \mathrm{~ns}$ delay on the Stop pulses to ensure that electrons are all measured with a positive value of $\tau_{\mathrm{TOF}}$ and, therefore, a valid coincidence.

The bias $V_{\mathrm{TOF}}$ of the TOF drift box is nominally $-11 \mathrm{kV}$ in ion mode and $+1.5 \mathrm{kV}$ in electron mode to accelerate the particles before they impact the foil. This mitigates the energy straggling (Allegrini et al. 2006) and angular scattering (Funsten et al. 1993) of the particles in the foil. This bias scheme also enables constant front and rear CEM detector biases, independent of whether ions or electrons are being measured. For each polar pixel, the Start and Stop CEMs are mounted into rectangular apertures in an electrode plate located opposite the drift box, as shown in Fig. 12. Both CEMs are biased at a fixed front voltage $V_{\text {CEM_F }}=+4.4 \mathrm{kV}$, such that secondary electrons generated at the start foil and the stop anode are accelerated into the Start and Stop CEM detectors, respectively. The CEM rear bias is nominally set to $V_{\text {CEM_R }}=+6.8 \mathrm{kV}$ and is adjustable up to a maximum of $+9.0 \mathrm{kV}$. The assembled TOF subsystem is shown in Fig. 13.

Considerable effort was directed toward the charged particle optics design of the TOF subsystem to efficiently steer and detect secondary electrons emitted only from the foil and anode surfaces, as well as to minimize detection of electrons from all other surfaces in the TOF subsystem. The preliminary optics design was described by MacDonald et al. (2009). Importantly, because (1) the electric field geometry that governs these secondary electron trajectories is solely defined by two electrodes (the TOF drift box at $V_{\mathrm{TOF}}$ and plate electrode at $\left.V_{\text {CEM_F }}\right)$, and (2) Start and Stop secondary electrons are born nearly at rest $(<10 \mathrm{eV})$ compared to the their acceleration energy $q\left(V_{\mathrm{CEM} \_\mathrm{F}}-V_{\mathrm{TOF}}\right)$ into the CEM detectors (minimum of $3.9 \mathrm{keV}$ in electron mode), the secondary electrons follow trajectories that are nearly 
Fig. $142.5 \mathrm{D}$ electron-optic simulations show the crossing trajectories of Start and Stop secondary electrons generated from the backside of the foil and from different locations along the Stop anode, respectively. The geometry was further refined using full 3D simulations. This geometry enabled efficient secondary electron collection at the Start and Stop CEM detectors using only two voltages, $V_{\mathrm{TOF}}$ and $V_{\mathrm{CEM} F}$, independent of the energy of the incident particle

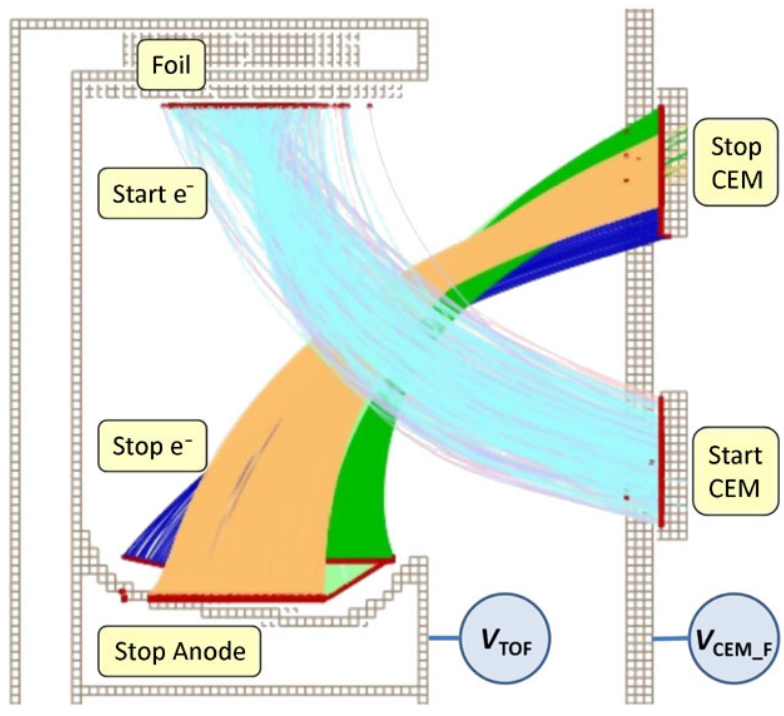

independent of $V_{\mathrm{TOF}}$, as demonstrated by subsystem testing and flight instrument calibration. This two-electrode design with crossing Start and Stop secondary electron trajectories enabled tremendous simplification of overall design and operation of the TOF subsystem. Furthermore, the separation of the polar angles into five independent polar pixels eliminates electronic cross-talk between pixels or crossing of secondary electron trajectories from one pixel to another.

The TOF subsystem was modeled with SIMION (Dahl 2000) in both 2.5-D and 3-D and validated through prototype testing and flight unit calibration. Charged particle optics optimization was performed through a complex parameter space, defining the foil dimensions, drift box dimensions, stop anode geometry, distance of CEM detectors and detector electrode plate from the drift box, and locations of the Start and Stop CEM detectors relative to the foil and stop anode, respectively. Figure 14 illustrates the trajectories of Start and Stop secondary electron trajectories for ion mode $\left(V_{\mathrm{TOF}}=-11 \mathrm{kV}, V_{\mathrm{CEM} \_\mathrm{F}}=+4.4 \mathrm{kV}\right)$ using a 2.5D electron-optic simulation with near-flight dimensions of the drift box geometry, CEM detector locations, and plate electrode to which the CEM detectors are attached. Start secondary electrons are emitted at energies of $0.2,2$, and $10 \mathrm{eV}$, while Stop secondary electrons are emitted at $2 \mathrm{eV}$; all are emitted within a half cone angle of $45^{\circ}$ relative to the emission surface normal. The secondary electrons follow trajectories from the Start foil and Stop anode that accurately map to the appropriate CEM detector and result in a collection efficiency $>90 \%$.

The TOF Subsystem development followed a methodical series of electron-optic modeling and validation steps. Full 3D electron-optic modeling was performed for incident $\mathrm{H}^{+}$, $\mathrm{He}^{+}, \mathrm{O}^{+}$, and electrons. For these simulations, the charged particles were initially input at specific locations of the foil to optimize the geometry of individual parts of the drift box; final simulations were performed using ion locations at the foil representative of the output from the ESA subsystem. Secondary electrons, which were generated where the ions exited the Start foil and struck the Stop anode, were modeled using a Gaussian energy distribution (with $2 \mathrm{eV}$ mean and $2 \mathrm{eV}$ FWHM) and random angular emission within a $45^{\circ}$ half cone angle relative to the local surface normal. In addition to optimization of the electron-optics 


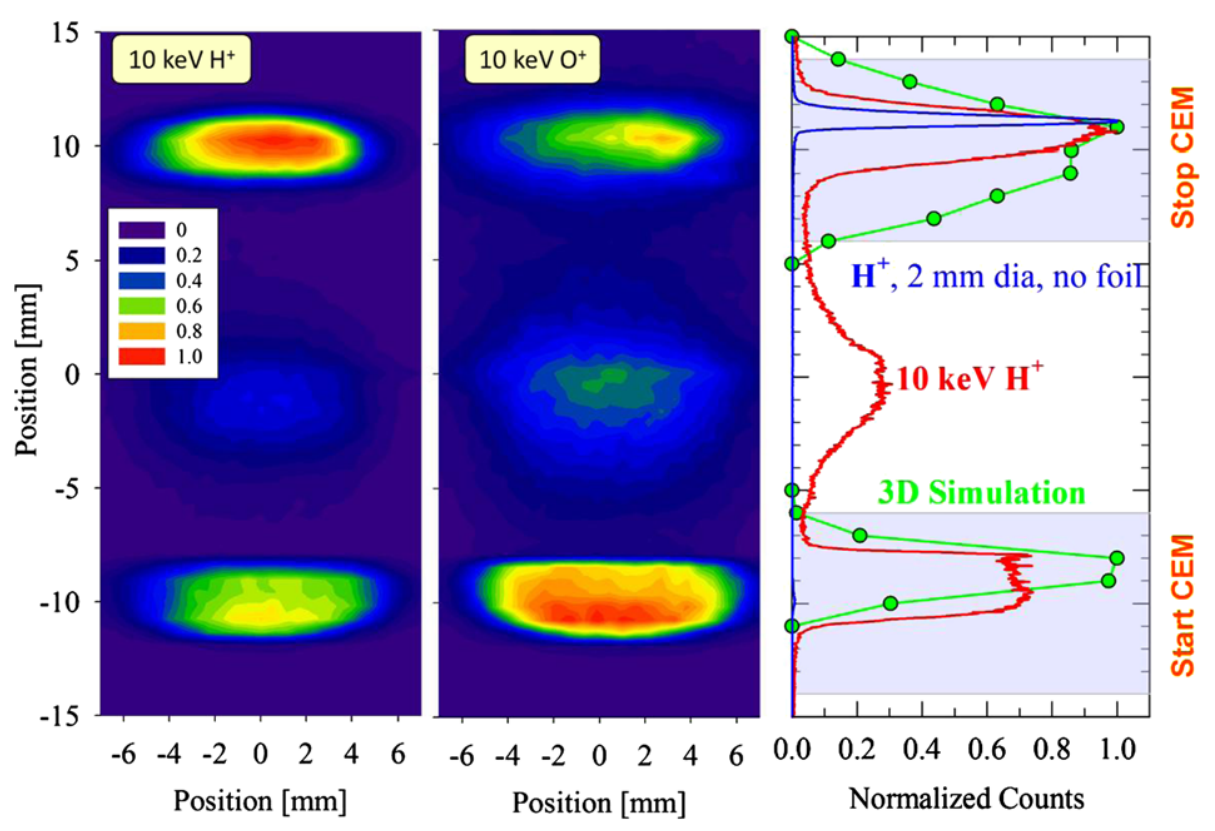

Fig. $153 \mathrm{D}$ electron-optic simulations were validated using a prototype polar pixel with an imaging MCP detector placed at the location of the CEM detectors. The left and center panels show the spatial distribution of normalized detected secondary electron counts for incident $10 \mathrm{keV} \mathrm{H}^{+}$(left panel) and $\mathrm{O}^{+}$(center panel) with $V_{\mathrm{TOF}}=-11 \mathrm{kV}$. The distributions at vertical positions centered near $-10 \mathrm{~mm}$ and $+10 \mathrm{~mm}$ correspond to Start and Stop secondary electrons, respectively. The right panel shows the vertical distribution of start and stop secondary electrons from incident $10 \mathrm{keV} \mathrm{H}^{+}$compared to electron-optic simulations in which the anode is broadly illuminated, showing the extent and positioning of collection required of the Stop CEM detector

of the TOF Subsystem, these models were used to estimate the TOF spectra for each of the charged particle species.

These simulations were initially validated using a polar pixel prototype with an imaging MCP detector placed at the location of the electrode plate to which the CEMs are attached. The left and center panels of Fig. 15 show the distribution of counts (normalized to unity) of secondary electrons emitted from the TOF drift box for incident $10 \mathrm{keV} \mathrm{H}^{+}$and $\mathrm{O}^{+}$for $V_{\mathrm{TOF}}=-11 \mathrm{kV}$. Distinct Start and Stop distributions are centered near vertical locations of $-10 \mathrm{~mm}$ and $+10 \mathrm{~mm}$, respectively, and each distribution lies within an area of $\sim 5 \mathrm{~mm}$ (vertical) $x \sim 12 \mathrm{~mm}$ (horizontal). The peaks observed in each panel near $0 \mathrm{~mm}$ vertical are artifacts of the resistive anode of the MCP detector, which centroids the coincident detection (within several $10 \mathrm{~s}$ of $\mathrm{ns}$ ) of start and stop electrons.

Figure 15, right panel, shows the vertical distribution of secondary electrons (red line) for the $10 \mathrm{keV} \mathrm{H}^{+}$prototype results in comparison with 3D SIMION simulation results for incident $1 \mathrm{eV} \mathrm{O}^{+}$(green points and line). For this worst-case simulation, the Stop anode is fully illuminated due to scattering of $\mathrm{O}^{+}$in the foil and results in a broader Stop secondary electron distribution. The funnel dimensions of the flight CEM detectors were $8 \times 20 \mathrm{~mm}$ to fully cover this area. The flight CEM detectors are located $19.6 \mathrm{~cm}$ apart (center-to-center). The blue line represents the counts (normalized to unity) from the prototype apparatus of a 2-mm-diameter $\mathrm{H}^{+}$beam with the Start foil removed so that Start secondary electrons are not generated. The resulting Stop peak is well defined because of no scattering of ions in the absence of the foil; note also the absence of the artifact peak near $0 \mathrm{~mm}$ vertical because no 
Start pulses (and therefore no Start and Stop coincidences that result in a centroid peak by the MCP resistive anode) are generated.

Further maturing the TOF subsystem design, the MCP detector was then replaced by a polar pixel assembly with CEM detectors, from which TOF spectra were derived and used for performance characterization and validation. A full 5-pixel subsystem was used to validate performance over all pixels, and in particular demonstrating similar performance of the two end polar pixels (Pixels 1 and 5) to the center polar pixel (Pixel 3) as well as negligible cross-talk between polar pixels.

3D SIMION simulations were also used for identifying the areas in the TOF box other than the Start foil and the Stop anode from which secondary and photo-electrons generated by penetrating radiation can or cannot access a CEM. Potential regions in which electrons generated by penetrating radiation might be focused to a CEM detector include the foil frame around the foil's exit surface, the back wall of the TOF drift box, and the side wall electrodes that separate each of the polar pixels. From this analysis, the side wall geometry was modified to a tab geometry (green sidewalls in Fig. 12) to eliminate electron emission from the side walls near the foil and Stop anode that could be steered to the CEM detectors. In the final design, only electrons emitted from the foil frame immediately adjacent to the exit surface of the foil and emitted from the back wall near the Stop anode could be steered to a CEM detector.

We describe each component of the TOF subsystem sequentially in more detail. Each polar pixel has a carbon foil centered at polar angles $0^{\circ}, \pm 36^{\circ}$, and $\pm 72^{\circ}$, with inner and outer radii of $60 \mathrm{~mm}$ and $68 \mathrm{~mm}$ and subtending $18^{\circ}$ in polar angle. These foils are nominal $0.6 \mu \mathrm{g} / \mathrm{cm}^{2}$ carbon from ACF Metals and are mounted on 333 line per inch (lpi) Ni grids. These grids have a typical transmission of $T_{\mathrm{G}}=70 \%$ as measured using a $50 \mathrm{keV} \mathrm{H}^{+}$beam and are attached to a stainless steel foil frame with $160 \mathrm{~mm}^{2}$ open area.

Foils of this thickness have extensive flight heritage (McComas et al. 2004) and are robust to sputtering at typical space plasma fluxes (Funsten and Shappirio 1997). Characterization of each flight foil included thickness measurement through light ion scattering (Funsten et al. 1992a) and pinhole characterization and quantification through heavy ion transmission (Funsten et al. 1992a, 1992b). Carbon foil thicknesses were measured within the range $1.5-1.8 \mu \mathrm{g} / \mathrm{cm}^{2}$ and averaged $1.6 \mu \mathrm{g} / \mathrm{cm}^{2}$, and each foil had less than $1 \%$ pinholes. The instrument performance model and electron-optic model incorporates foil effects on ions including angular scattering (Funsten et al. 1993), exit charge state (Funsten et al. 1993), and energy loss (Allegrini et al. 2006). Interactions of incident electrons with the foil were simulated using CASINO (Drouin et al. 2007; Demers et al. 2011).

We note that the RBSP mission poses a potential risk to high flux, reactive ion etching of the ultrathin carbon foils near the $\sim 600 \mathrm{~km}$ altitude perigee at which the $\mathrm{O}^{+}$density can reach $10^{5} \mathrm{~cm}^{-3}$ (Nosé et al. 2009). Reactive ions such as $\mathrm{O}^{+}$can rapidly etch hydrocarbons, at a much higher rate than physical sputtering by inert ions, by forming volatiles such as $\mathrm{CO}$ and $\mathrm{CO}_{2}$ (Oehrlein et al. 2011); we similarly expect enhanced etch rates of carbon foils from low energy $\mathrm{O}^{+}$. The RBSP spacecraft speed approaches $10 \mathrm{~km} / \mathrm{s}$ at perigee, thus encountering an $\mathrm{O}^{+}$ram flux of $10^{11} \mathrm{~cm}^{-2} \mathrm{~s}^{-1}$ and $\mathrm{O}^{+}$ram energy of $\sim 8.2 \mathrm{eV}$. Considering spacecraft charging up to $-1.5 \mathrm{~V}$ at this altitude (Anderson et al. 1994), care must be taken to ensure that HOPE does not measure ions less than $10 \mathrm{eV}$ in the vicinity of perigee so that the foils in the TOF subsystem are not exposed to the large $\mathrm{O}^{+}$flux. Because of the need to avoid this intense $\mathrm{O}^{+}$flux near perigee, HOPE nominally operates with a minimum energy of $1 \mathrm{eV}$ in ion mode and $15 \mathrm{eV}$ for electron mode except during a perigee segment (within $\pm 20 \mathrm{~min}$ of perigee, below $\sim 3500 \mathrm{~km}$ altitude) in which the minimum energy is at least $15 \mathrm{eV}$ for both ions and electrons. 
Fig. 16 The Stop anode shape was optimized to minimize the variation of the pathlength of particles in the drift section due to angular scattering in the foil and to maximize the probability that Stop secondary electrons are steered into the Stop CEM detector

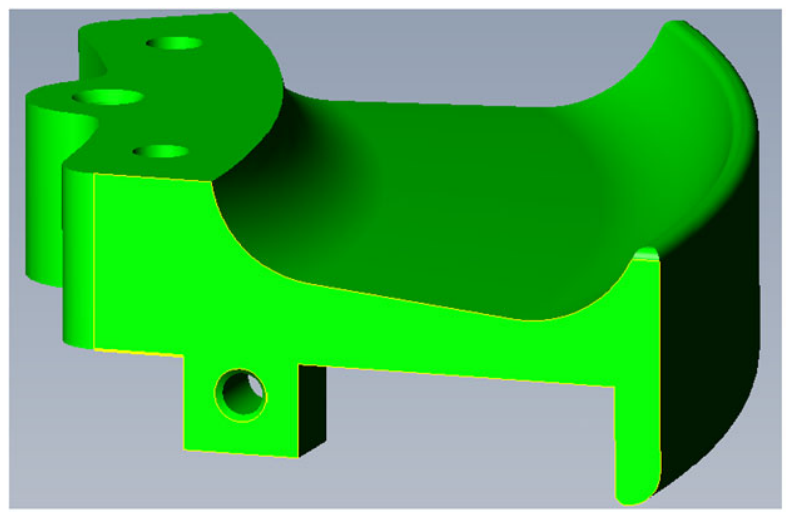

In ion mode, Start electron production is the result of forward secondary electron emission from the carbon foil, with a mean forward secondary electron yield $\gamma_{\mathrm{F}}=0.61 E^{0.56}$ for $\mathrm{H}^{+}$incident at energy $E$ in $\mathrm{keV}$ (Ritzau and Baragiola 1998). For heavier ions at the same energy, the forward secondary electron emission yields are higher. The probability of a nonzero number of electrons emitted can be calculated using a Poisson distribution. In electron mode, due to the lack of studies of electrons transiting carbon foils yields, we approximate the yield using published data of electrons incident on thick targets (e.g., Seiler 1983).

Relative to the HOPE axis of cylindrical symmetry, the envelope of each TOF drift box is $54 \mathrm{~mm}$ radius inner wall, $74 \mathrm{~mm}$ outer radius (foil frame and Stop anode), and spans $\sim 36^{\circ}$. Partial walls were added between each of the polar pixels to push secondary electrons generated at the outer edges of the foil and Stop anode toward the appropriate CEM detector as well as to provide a barrier to prevent incident ions and electrons that are highly scattered at the foil in one pixel from reaching another pixel. The walls are strategically located and of minimum size to minimize the surface area from which penetrating radiation can generate electrons that might be detected as background.

The Stop anode, shown schematically in Fig. 16, was fabricated using Al 6061, whose native aluminum oxide has been shown to enhance secondary electron emission for incident electrons (Walker et al. 2008) and ions (Dietz and Sheffield 1975; Baragiola et al. 1979). We expect that the native oxide will remain stable far beyond the nominal RBSP mission. The shape of the anode was initially a spherical section with vertex in the middle of the foil to minimize the variation of pathlength of incident particles (and therefore minimize TOF variation of incident particles) in the drift section due to angular scattering in the foil. This general shape was subsequently modified to enhance the electron-optic steering of Stop secondary electrons to the Stop CEM, primarily by introducing a small tilt of the surface toward the Stop CEM detector and a flatter bottom.

Each polar pixel has separate Start and Stop CEM detectors. The CEM detectors, Sjuts KBL series, have a rectangular funnel with an entrance dimension $8 \mathrm{~mm} \times 20 \mathrm{~mm}$. All CEMs were burned in to $\sim 0.1 \mathrm{C}$ output charge to screen for infant mortality. The CEMs are mounted in rectangular holes in the detector electrode plates; both the CEM fronts and the electrode plates were biased to the same potential $V_{\text {CEM_F }}$ that is fixed to $+4.4 \mathrm{kV}$ using three separate shunt regulators attached to Pixels $1 \& 4$, Pixels, $2 \& 5$, and Pixel 3. In front of each CEM funnel is a 20 line per inch nickel grid to suppress secondary electrons generated in the funnel. CEMs were selected and matched as pairs for each polar pixel based similarity of gains measured during CEM burn-in as well as similarity of electrical impedance. The gain curves derived before final instrument delivery are shown in the Calibration section. 
Fig. 17 Electronic shielding within and between polar pixels was found to reduce the level of electronic cross-talk of sufficient magnitude to register noise counts. The intra-pixel shield consisted of a copper sheet sandwiched within Kapton that surrounded a CEM detector as shown in the figure. The inter-pixel shield consisted of an aluminum plate between the polar pixels

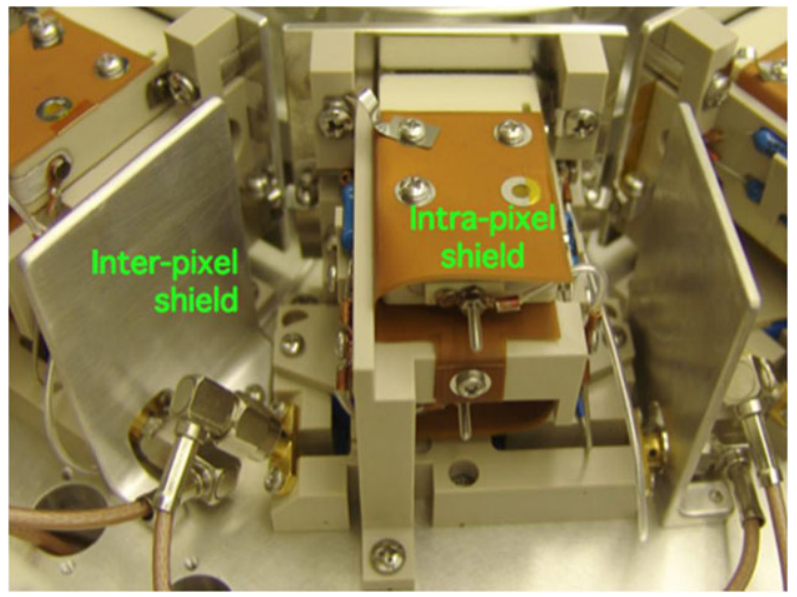

Testing of CEMs was performed to assess intrinsic detector timing jitter, which is important for understanding TOF performance limitations and is used to appropriately design the drift box geometry and TOF electronics. An ion beam was directed on a thin carbon foil, generating secondary electrons simultaneously from the entrance surface, which were detected by one CEM detector, and exit surface, which were detected by a second CEM detector. Because electron transport from foil to detector is much shorter than CEM timing jitter, the time difference between detected coincidences provides a measure of the timing uncertainty of the CEM detectors. A delay line was inserted into the electronic chain of one CEM so that all coincidences registered a positive timing difference. The FWHM of this timing difference was found to be $3.5 \mathrm{~ns}$ FWHM, which was used to define the drift box length and the required TOF electronics timing accuracy for each polar pixel.

Electronic cross talk was minimized with inter- and intra-pixel shields as shown in Fig. 17. The intra-pixel shield is a sandwiched layer of copper and Kapton to provide grounded signal isolation. Particle crosstalk between pixels was measured with a low energy, heavy ion beam $\left(5 \mathrm{keV} \mathrm{O}^{+}\right)$to maximize angular scattering from the foil and therefore maximize the leakage of scattered particles into adjacent polar pixels. No particle crosstalk was observed as will be shown by the polar angle response in the Calibration section.

The performance of the TOF subsystem is governed by numerous complex physical processes, including angular scattering and energy loss of incident charged particles in the foil, secondary electron emission statistics from the foil and the Stop anode, the steering of Start and Stop secondary electrons into the appropriate CEM detector, and probability of detection of these secondary electrons. This ensemble of processes is difficult to model and can change over time, so the subsystem performance model relies heavily on the extensive test and calibration data acquired throughout instrument development and interpolation of these empirical results over energy, angle, and species. We also report as routine HOPE data products both coincidence and non-coincidence count rates from which we monitor the absolute detection efficiencies of each polar pixel of the TOF subsystem over the lifetime of the mission.

\subsubsection{Penetrating Radiation Analysis}

The fundamental challenge for measuring the plasma environment in the heart of the radiation belts is minimizing the background from the high flux of penetrating radiation. As 
previously discussed, HOPE incorporates several strategies to mitigate this background. Of these, shielding has the highest impact for removing this background. Because the penetrating radiation flux exponentially decreases with shielding thickness, small spots of thin shielding can potentially compromise the diligent shielding in all other areas. Therefore, extreme care was taken in the mechanical design to prevent this type of compromise, for example using step mechanical interfaces, strategically locating screw vent holes in any parts used for shielding, and spot shielding key areas identified through simulations.

An empirical scaling analysis compared background count rates from the Magnetospheric Plasma Analyzer (MPA) on LANL satellites (Bame et al. 1993) in geosynchronous orbit to the ambient penetrating particle radiation environment in the radiation belts to develop a basis for estimating detector background count rates over the RBSP orbit (MacDonald et al. 2006). Like HOPE, MPA used CEM detectors, although not in a coincidence configuration. Signal-to-background coincident and non-coincident rates were estimated for HOPE based on scaling of the orbit characteristics, instrument response functions, detector response, and coincident timing window. These results were then used as a basis for simulation studies using a geant 4 model (Agostinelli et al. 2003) to understand the susceptibility of HOPE to penetrating radiation. Two studies were performed during development of HOPE design, and a final analysis was performed for verification of the flight design, from which we derived an expected accidental coincidence rate of $\sim 1 \mathrm{~Hz}$. The analysis for the flight instrument design was validated by agreement of the simulated flight instrument response to its measured response from exposure to a $15 \mathrm{mCi}^{60} \mathrm{Co} \gamma$-ray source during calibration.

Decay of materials activated by $\mathrm{MeV}$ protons can induce backgrounds in instruments (e.g., Väyrynen et al. 2009). HOPE activation analysis focused on materials within the HOPE instrument and, especially, candidate materials for external wall shielding. Slugs of stainless steel, tungsten copper, tantalum, aluminum, G11 fiberglass, copper, and $0.89 \mathrm{~cm}$ aluminum shielded copper were exposed over $\sim 30$ min to protons from 10-200 MeV in $25 \mathrm{MeV}$ steps using the Alternating Gradient Synchrotron (AGS) (Lowenstein and Rusek 2007) at the NASA Space Radiation Laboratory (NSRL) at Brookhaven National Laboratory (BNL). The proton fluence was the equivalent of one orbit exposure as derived from the AP-8 model (Sawyer and Vette 1976) at worst case at the RBSP orbit. The only material exhibiting any long-term activation was copper, which took $\sim 3$ hours to decay to background levels, confirming that significant amounts of copper should be avoided. Stainless steel showed a similar initial specific activity after irradiation, but decayed to background levels in $\sim 10 \mathrm{~min}$; we therefore anticipate slightly enhanced background for up to $\sim 10 \mathrm{~min}$ after exiting of the inner belt proton population.

Sensitivity analyses were performed during the design stage and for verification of statistically significant measurements during worst-case flux scenarios in the radiation belts. These analyses used the Sector Shielding Analysis Tool (SSAT) (Santina et al. 2003) based on geant 4 . The simulated instrument geometry incorporated key HOPE components including all exterior walls, the interface plate between the TOF subsystem and the HEU, and the ESA subsystem. Screws in these components were assumed to uniformly fill their tapped holes with the same material as the component itself. Most small components (e.g., detectors, foils, wires, individual electrical components, door mechanism) and components with comparatively small mass shielding (HEU electronic boards, detector mounting brackets, connectors) were not included in the geometry model. Additionally, the analyses assumed no shielding contribution for the spacecraft, so the analyses represent a worst-case shielding mass density.

The design stage sensitivity analysis derived the shielding mass density integrated along lines-of-slight (LOS) distributed over the complete $4 \pi$ sr solid angle at various interior locations, primarily in the TOF Subsystem and the HEU. This analysis verified the RBSP 
Fig. 18 Ambient penetrating electron spectrum used to characterize worst-case fluxes of penetrating radiation for the RBSP mission

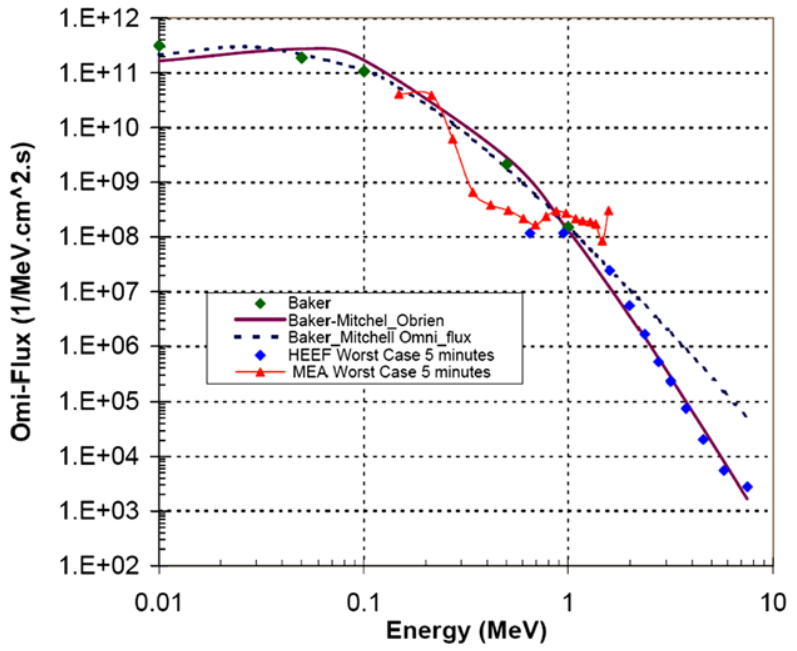

shielding requirement of $\geq 0.89 \mathrm{~cm}(0.350$ in) aluminum equivalent thickness between any electronics and the instrument exterior.

Another important result was identification and resolution of a set of penetrating particle trajectories that could traverse both CEM detectors of a single polar pixel. Such a particle could register a valid event with a short TOF that may masquerade as detection of a plasma electron or a fast, light ion such as $50 \mathrm{keV} \mathrm{H}^{+}$. The HEU was found to provide sufficient shielding for penetrating radiation that traverses the Start CEM before the Stop CEM. For penetrating radiation entering HOPE from the opposite direction, traversing the Stop CEM before the Start CEM, the thickness of portions of the exterior wall in the Door subsystem was increased to nearly $1.8 \mathrm{~cm}(\sim 0.7$ in) aluminum. Additionally, the top of the top-hat ESA on which the door actuator is mounted was changed from aluminum to stainless steel for enhanced shielding with economy of space. We found through simulation with anticipated worst-case radiation belt spectra that the additional thickness blocked almost all penetrating protons or electrons that would otherwise transit the two CEMs of a single polar pixel.

The sensitivity analysis verification evaluated the HOPE penetrating background based on RBSP project-developed spectra. The electron spectrum encompasses the CRRES High Energy Electron Fluxmeter (HEEF) worst case 5 minutes and CRRES Medium Electrons A (MEA) worse case 5 minutes for electrons (Fig. 18). The composite electron spectra, known as Baker-Mitchell-O'Brien worst-case, was constructed by the RBSP project for verification processes across all instruments and subsystems. The proton spectra are derived from the AP-8 peak spectrum. The same HOPE geometry model was used as in the design stage sensitivity analysis, but without the shielding added to minimize radiation that would traverse the two CEMs of a single polar pixel. The simulations derived a maximum $0.7 \mathrm{~Hz}$ coincidence count rate from penetrating electrons and ions combined for any polar pixel assuming random coincidence within the $160 \mathrm{~ns}$ TOF window in ion mode. We project this background count rate into an equivalent ambient plasma flux outside the instrument using the maximum HOPE geometric factor. This flux can then be compared to a typical stormtime flux, e.g., Krimigis et al. (1985). Figure 19 shows significant (more than an order of magnitude) separation between the anticipated plasma ion signals and the penetrating background signal expected in the HOPE instrument over all except the highest energy $\mathrm{He}^{+}$and $\mathrm{O}^{+}$, for which the separation remains nearly a factor of 10 . Considering that most assumptions for 
Fig. 19 Penetrating electrons and ions combine to produce background counts in HOPE that might be improperly identified as real plasma ions or electrons. We project the simulated worst-case background count rate from penetrating ions and electrons to an equivalent ion flux at the HOPE entrance aperture (green line). Comparison of this background flux with typical stormtime fluxes for $\mathrm{H}^{+}$(black line), $\mathrm{He}^{+}$(blue line), and $\mathrm{O}^{+}$ (red line) from Krimigis et al. (1985) shows that backgrounds from penetrating radiation should be negligible

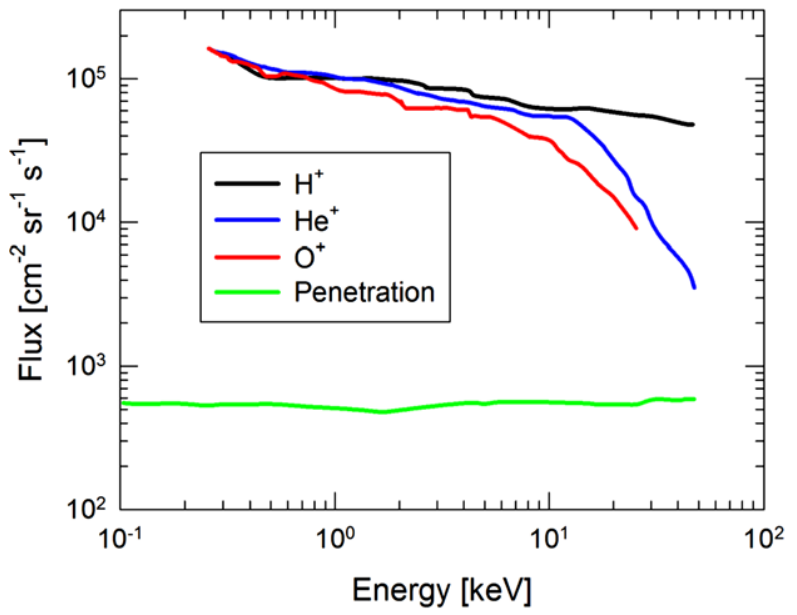

this analysis are worst-case, penetrating background is not expected to be the major source of noise in the HOPE measurements.

\subsection{HOPE Electronics Unit (HEU)}

The HOPE Electronics Unit (HEU) is multipurpose, designed to communicate with the spacecraft; generate appropriate voltages for operation; receive analog signals from the TOF subsystem and process them into HOPE data products; and monitor housekeeping. The HEU is divided into the following functional elements, shown in Fig. 20: the Low Voltage Power Supply (LVPS) Board that generates conditioned power for the HEU, the High Voltage Power Supply (HVPS) Board that generates high voltages for charged particle optics elements and for detector bias; the Front End Electronics (FEE) Board that receives analog signals from the CEM detectors and converts them into digital signals; and the Digital Board (DB) that processes these digital signals and classifies them as detected events. In addition, the Backplane (BP) serves as the primary communications and interface trunk for all of the HEU boards as well as with the HOPE door and the spacecraft. The HEU interfaces with the spacecraft for $+30 \mathrm{~V}$ primary power and low-voltage differential signaling (LVDS) communications, which consists of three pairs of interfaces: inbound synchronization, inbound command signals, and outbound telemetry. The synchronization signal from the spacecraft provides both a one-pulse-per-second (1-PPS) signal and a spin pulse signal. We first provide an overview of each board and then provide an overview of HEU operational modes.

\subsubsection{Power Supply Boards}

The LVPS Board receives spacecraft power $(+30 \mathrm{~V})$ and converts it to the following secondary voltages needed throughout the HEU: $+3.3 \mathrm{~V}, \pm 5 \mathrm{~V}, \pm 12 \mathrm{~V}$. The main components are three International Rectifier DC-to-DC converters, one filter, and a slow-start circuit. As with every HEU electronics board, an AD590 temperature transducer is included to monitor temperature. The LVPS Board is mounted directly on the bottom of the HOPE chassis, providing a strong thermal conduction path to the spacecraft. The layout and grounding design is in accordance with RBSP mission requirements with special attention paid to minimizing current loop areas and utilizing a star ground. 


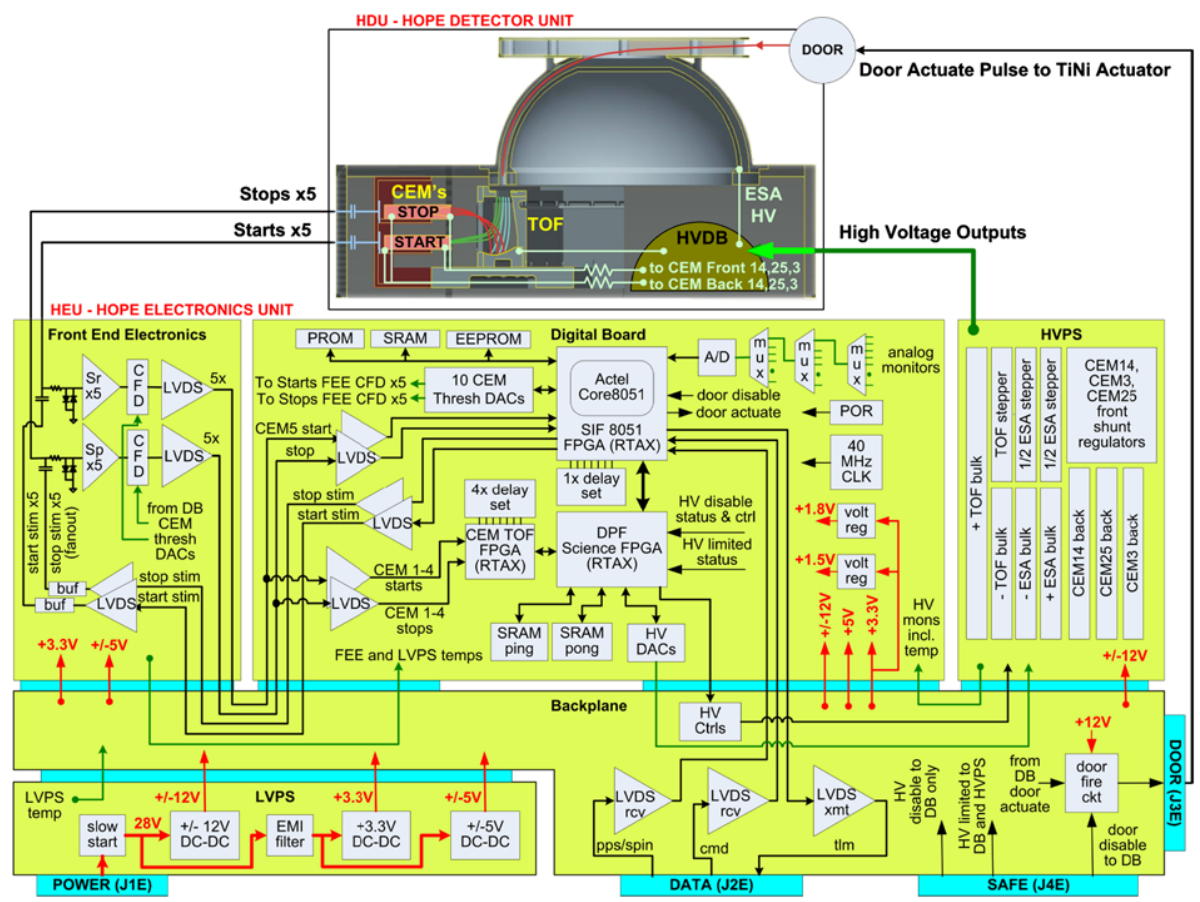

Fig. 20 Block diagram of the Hope Electronics Unit (HEU)

The HVPS Board, powered by $\pm 12 \mathrm{~V}$, provides bipolar ESA $\left(V_{\mathrm{ESA}}\right)$ and TOF $\left(V_{\mathrm{TOF}}\right)$ voltages, as well as CEM bias voltages $V_{\text {CEM_F }}$ and $V_{\text {CEM_R }}$. To minimize the effect of either a possible $\mathrm{HV}$ or CEM failure, three separate CEM supplies provide voltages to polar pixels $1 \& 4,2 \& 5$, and 3 . The CEM front voltages $V_{\text {CEM F }}$ are obtained from the same supply as the rear voltages $V_{\text {CEM_R }}$ using a shunt regulator circuit that maintains a fixed $+4.4 \mathrm{kV}$ level. To provide the required voltage resolution over the wide dynamic range of HOPE's energy response ( $1 \mathrm{eV}$ to $50 \mathrm{keV}$ ), the ESA supply is dual-range, with low range extending over 0-33 V and high range spanning 0-7500 V. Both the ESA and TOF supplies are bipolar, allowing ion and electron measurements in alternating spins using the same supplies.

HVPS board inputs consist of a series of high-low enables: one overall enable; one for each of the three CEM supplies; and one for each of the following: $\operatorname{ESA}(+), \operatorname{ESA}(-)$, ESA high/low range, $\mathrm{TOF}(+)$, and $\mathrm{TOF}(-)$. Also included are digital-to-analog converters (DAC) to control the voltage levels of the three CEM supplies, the ESA supply, and the TOF supply. Scaled analog voltage monitors are provided for all the high-voltage outputs and bulk supplies, along with current monitors for the outputs of the three CEM supplies The supplies use a resonant flyback topology running in the range 100 to $125 \mathrm{kHz}$. Custom lug connections are used at the detector via a high-voltage distribution block. A V/10 function enabled by an external hardware plug limits the output of the high voltage power supplies to a level safe for limited instrument functionality testing at atmospheric pressure. The largest ESA step, between $4077 \mathrm{~V}$ and $6440 \mathrm{~V}$, occurs over $\sim 0.6 \mathrm{~ms}$, which is well within the required transition time of $1.3 \mathrm{~ms}$. The HVPS specifications are summarized in Table 3 . All supplies except for the bulk supplies have 12 bit DAC resolution. Table 4 shows additional information for the ESA and TOF stepping supplies. 
Table 3 Summary of HOPE high voltage power supply (HVPS) specifications

\begin{tabular}{|c|c|c|c|c|c|}
\hline \multirow{2}{*}{$\begin{array}{l}\text { HVPS } \\
\text { name }\end{array}$} & \multirow{2}{*}{$\begin{array}{l}\text { HVPS } \\
\text { type }\end{array}$} & \multicolumn{2}{|c|}{ Voltage (kV) } & \multirow{2}{*}{$\begin{array}{l}\text { Max current } \\
(\mu \mathrm{A})\end{array}$} & \multirow{2}{*}{$\begin{array}{l}\text { Voltage/current } \\
\text { monitor }^{\mathrm{b}}\end{array}$} \\
\hline & & Min & $\overline{\operatorname{Max}}$ & & \\
\hline ESA Bulk (+) & Fixed & 0 & +8.0 & & $\mathrm{Y} / \mathrm{N}$ \\
\hline ESA Bulk (-) & Fixed & -8.0 & 0 & & $\mathrm{Y} / \mathrm{N}$ \\
\hline $\operatorname{ESA}\left(V_{\mathrm{ESA}}\right)$ & Stepping $^{\mathrm{a}}$ & -7.5 & +7.5 & & $\mathrm{Y} / \mathrm{N}$ \\
\hline TOF Bulk (+) & Fixed & 0 & +2.0 & & $\mathrm{Y} / \mathrm{N}$ \\
\hline TOF Bulk (-) & Fixed & -11.5 & 0 & & $\mathrm{Y} / \mathrm{N}$ \\
\hline TOF $\left(V_{\mathrm{TOF}}\right)$ & Stepping $^{\mathrm{a}}$ & -11.0 & +1.5 & & $\mathrm{Y} / \mathrm{N}$ \\
\hline $\begin{array}{l}\left.\text { CEM Front ( } V_{\text {CEM_F }}\right) \text {, } \\
\text { Polar pixels } 1 \& 4\end{array}$ & Fixed & 0 & +4.4 & 120 & $\mathrm{Y} / \mathrm{Y}$ \\
\hline $\begin{array}{l}\left.\text { CEM Front ( } V_{\text {CEM_F }}\right) \text {, } \\
\text { Pixels } 2 \& 5\end{array}$ & Fixed & 0 & +4.4 & 120 & $\mathrm{Y} / \mathrm{Y}$ \\
\hline $\begin{array}{l}\left.\text { CEM Front ( } V_{\text {CEM_F }}\right) \text {, } \\
\text { Pixel } 3\end{array}$ & Fixed & 0 & +4.4 & 60 & $\mathrm{Y} / \mathrm{Y}$ \\
\hline $\begin{array}{l}\left.\text { CEM Rear ( } V_{\text {CEM_R }}\right) \text {, } \\
\text { Pixels } 1 \& 4\end{array}$ & Programmable $^{\mathrm{a}}$ & 0 & +9.0 & 120 & $\mathrm{Y} / \mathrm{Y}$ \\
\hline $\begin{array}{l}\left.\text { CEM Rear ( } V_{\text {CEM_R }}\right) \text {, } \\
\text { Pixels } 2 \& 5\end{array}$ & Programmable ${ }^{a}$ & 0 & +9.0 & 120 & $\mathrm{Y} / \mathrm{Y}$ \\
\hline $\begin{array}{l}\left.\text { CEM Rear ( } V_{\text {CEM_R }}\right) \text {, } \\
\text { Pixel } 3\end{array}$ & Programmable ${ }^{\mathrm{a}}$ & 0 & +9.0 & 60 & $\mathrm{Y} / \mathrm{Y}$ \\
\hline
\end{tabular}

aprogramming voltages are $\pm 5 \mathrm{~V}$ (bipolar) or $0 \mathrm{~V}$ to $+5 \mathrm{~V}$ (unipolar) full scale

${ }^{\mathrm{b}}$ Monitor voltages are $\pm 4 \mathrm{~V}$ (bipolar) or $0 \mathrm{~V}$ to $+4 \mathrm{~V}$ (unipolar) full scale voltage

Table 4 Additional specifications for HOPE stepping high voltage power supplies

\begin{tabular}{llllll}
\hline $\begin{array}{l}\text { Stepping } \\
\text { supply }\end{array}$ & $\begin{array}{l}\text { Min/max } \\
\text { voltage }\end{array}$ & $\begin{array}{l}\text { Settling } \\
\text { time }\end{array}$ & $\begin{array}{l}\text { Time } \\
\text { per step }\end{array}$ & $\begin{array}{l}\text { Max step } \\
\text { change }\end{array}$ & $\begin{array}{l}\text { Total load } \\
\text { capacitance }\end{array}$ \\
\hline ESA & $-7.5 \mathrm{~V} /+7.5 \mathrm{kV}$ & $1.3 \mathrm{~ms}$ & $10.4 \mathrm{~ms}$ & $1.9 \mathrm{kV}$ & $150 \mathrm{pF}$ \\
TOF & $-11.0 \mathrm{kV} /+1.5 \mathrm{kV}$ & $10.4 \mathrm{~ms}$ & $12 \mathrm{~s}$ & $12.5 \mathrm{kV}$ & $100 \mathrm{pF}$ \\
\hline
\end{tabular}

\subsubsection{Front End Electronics (FEE) Board}

The FEE Board is powered by $\pm 5 \mathrm{~V}$ and $+3.3 \mathrm{~V}$, with the anode outputs of the five CEM pairs (10 channels total) of polar pixels as its primary functional inputs. Each CEM output signal connects to an SMA connector on the FEE and is followed by a zap-trap, comprised of a reversed pair of zener diodes to ground for sinking voltage transients. The signal chain continues with an AD8001 charge amplifier, a constant fraction discriminator (CFD), and digital output to the DB. Each channel has its own isolated reference plane to minimize electrical cross-talk between them. An extra $10 \mathrm{~ns}$ delay is added to each of the five Stop CEM channels to ensure that a Stop pulse is preceded in time by its correlated Start pulse, which is especially important in electron mode in which incident electrons produce an extremely short $\tau_{\mathrm{TOF}}$. Each charge amplifier has its own analog threshold to provide individual control over a dynamic range of 100; this allows for changes in level sensitivity over the mission for each CEM. Once a threshold is crossed, a channel is activated, and the CFD then provides 
triggering at a given percentage level (e.g., $50 \%$ ) so that a consistent level crossing of the CEM signal is used for the time-of-flight measurement.

The DB can be commanded to inject artificial pulses into the input of each FEE channel so that end-to-end functional tests of the full electronics chain can be performed without requiring operation of the CEM detectors. The repetition rate and relative timing of pulses injected into correlated Start-Stop channel pairs are both configurable. Each FEE channel demonstrated the processing of periodic pulses exceeding a preiodic injection rate of $2 \mathrm{MHz}$ without pulse pileup. The electronics timing jitter is $\leq 1 \mathrm{~ns}$ FWHM for each channel.

\subsubsection{Digital Board}

The DB is powered by $\pm 12 \mathrm{~V},+5 \mathrm{~V}$, and $+3.3 \mathrm{~V}$. It receives spacecraft commands, the synchronization pulse, temperature monitors from all boards, HVPS voltage and current monitors, FEE signals, and status signals from the V/10 plug and door interface. The DB outputs are: telemetry to the spacecraft, digital-to-analog converter (DAC) analog signals as input for the HVPS and FEE thresholds, HVPS enable signals, and the door firing signal. The DB is driven by a $40 \mathrm{MHz}$ oscillator which is the primary clock throughout the digital system. The DB contains a $32 \mathrm{kB}$ PROM for the permanent storage of bootup flight software, $128 \mathrm{kB}$ EEPROM for non-volatile (yet in-flight re-writeable) storage of application flight software and tables, $512 \mathrm{kB}$ SRAM for flight software use during code execution, and $2 \times$ $512 \mathrm{kB}$ SRAM for science data acquisition buffering. Three FPGAs reside on the DB: the Spacecraft Interface (SI) FPGA, the Data Processing (DP) FPGA and the Time-of-Flight (TOF) FPGA. The SI FPGA contains an 8051 core which runs the flight software (FSW), executing at 5 million instructions per second (MIPS). The SI FPGA contains first-in-firstout (FIFO) resources for inbound and outbound communications with the spacecraft. The DP FPGA controls the ESA stepping and ESA and TOF polarity changes during nominal science acquisition, which is illustrated in Figs. 21 and 22.

Because HOPE requires a constant time cadence for stepping through the 72 energy steps, HOPE data acquisition is not synchronized to the spacecraft spin pulse. Instead, acquisition is defined by internal timing, which is an important function for the DB. The time $\tau_{\mathrm{ESA}}$ STEP spent at a single ESA level represents the fundamental time cadence for all HOPE data acquisition. As shown in Fig. 21, $\tau_{\text {ESA_STEP }}$ is constructed from a high voltage settling time during which data is not recorded and a science acquisition period during which data is recorded. Both of these can be independently adjusted as needed and are nominally set to $1.302 \mathrm{~ms}$ and $9.115 \mathrm{~ms}$, respectively. The nominal value of $\tau_{\text {ESA_STEP }}$ is therefore $10.417 \mathrm{~ms}$ and can be adjusted in flight over a limited range to allow the HOPE measurement interval to approximately match the final spacecraft spin period.

As shown in Fig. 21 for normal science mode, each ESA sweep of 72 steps constitutes a single azimuthal bin over $72 \times \tau_{\text {ESA_STEP }}=750 \mathrm{~ns}$. As shown in Fig. 22, 16 ESA sweeps over one spacecraft spin constitute a complete electron or ion frame with 16 azimuthal bins acquired for each polar pixel, corresponding to $22.5^{\circ}$. Based on $\tau_{\text {ESA_STEP }}=10.417 \mathrm{~ms}$, an electron or ion frame is acquired every $12 \mathrm{sec}$, and a Master Frame of one electron frame and one ion frame is acquired every $24 \mathrm{sec}$. The spacecraft spin pulse timestamp is correlated with the ion and electron frame timestamps to precisely define the azimuthal look direction within each frame. We note that the settling time of the TOF HVPS between electron and ion frames is also adjustable up to $100 \mathrm{~ms}$, if needed.

The TOF FPGA, under the control of the DP FPGA, collects and processes the CEM pulses from each polar pixel at each ESA step. The TOF FPGA classifies each detected event and records events in eight event counters. The TOF measurement concept and its 


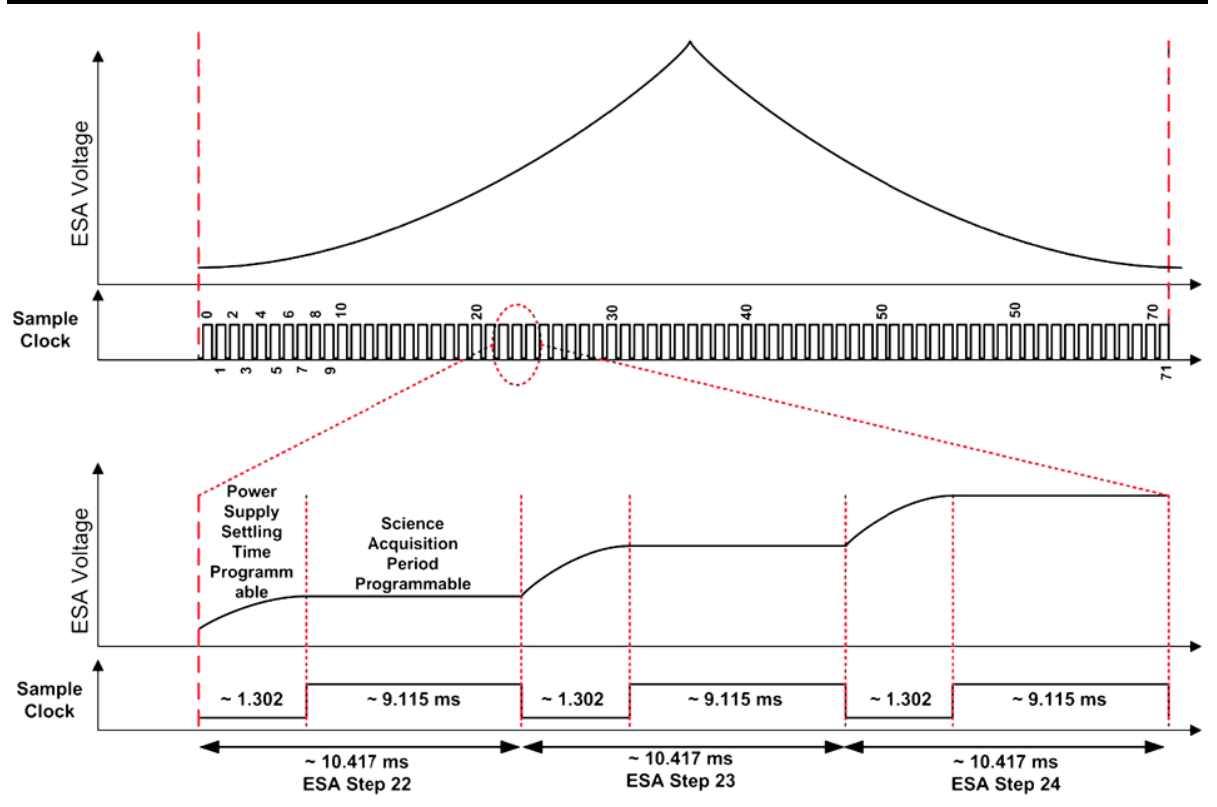

Fig. 21 Timing diagram for one ESA sweep, which traverses 72 discrete energy steps. Each ESA step is nominally $\tau_{\text {ESA_STEP }}=10.417 \mathrm{~ms}$, over which the voltage is allowed to settle for $1.302 \mathrm{~ms}$ and data is acquired for the remaining $9.115 \mathrm{~ms}$. Both of these timing values are adjustable, and the net value $\tau_{\text {ESA STEP }}$ represents the fundamental timing period for acquisition of all HOPE data. A single ESA voltage sweep based on $\tau_{\text {ESA_STEP }}=10.417 \mathrm{~ms}$ is $750 \mathrm{~ms}$

implementation for a single polar pixel with a pair of CEM detectors are shown in Fig. 23. The block diagram (top panel) shows the Start and Stop signals output from the CEMs, represented as Gaussian pulses in the timing diagram. These signals are converted by the FEE to the Start and Stop digital signals. Because of intrinsic timing error within the system, e.g., CEM timing jitter, a $10 \mathrm{~ns}$ delay is added to the Stop signal to ensure that a Stop pulse always follows the Start pulse, which is needed for fast particles such as $50 \mathrm{keV} \mathrm{H}^{+}$and electrons. The FEE also lengthens the digital signals to $\sim 90 \mathrm{ns,} \mathrm{which} \mathrm{are} \mathrm{easier} \mathrm{for} \mathrm{the}$ FPGA to process.

The TOF FPGA calculates the elapsed time between the arrivals of the Start and Stop pulses with $2.5 \mathrm{~ns}$ time resolution using an innovative approach that does not require the resources needed for traditional precision timing. First, a coarse time measurement $\tau_{\text {COARSE }}$ is derived (Fig. 23, bottom panel) by counting the number of elapsed $25 \mathrm{~ns}$ periods of a $40 \mathrm{MHz}$ clock between the clock's first rising edges after receipt of a Start pulse and after receipt of a Stop pulse. Two fine timing values are then derived: the time $\tau_{\text {FINE_ST }}$ between the receipt of the Start pulse and the start of the first clock pulse, and time $\tau_{\text {FINE_SP }}$ between the receipt of the Stop pulse and the start of the last clock pulse. Using this scheme, the measured TOF is derived by adding $\tau_{\text {FINE_ST }}$ to $\tau_{\text {COARSE }}$ and subtracting $\tau_{\text {FINE_SP }}$ from $\tau_{\text {COARSE }}$. The particle TOF is therefore

$$
\tau_{\mathrm{TOF}}=\tau_{\text {FINE_ST }}+\tau_{\mathrm{COARSE}}-\tau_{\mathrm{FINE} \_\mathrm{SP}}-10 \mathrm{~ns}
$$

where the $10 \mathrm{~ns}$ delay line added to the Stop signal is also included.

The fine timing is enabled by inputting the Start or Stop signal into a delay line with 10 taps at intervals of $2.5 \mathrm{~ns}$. As the signal propagates with time along the tapped delay line, 

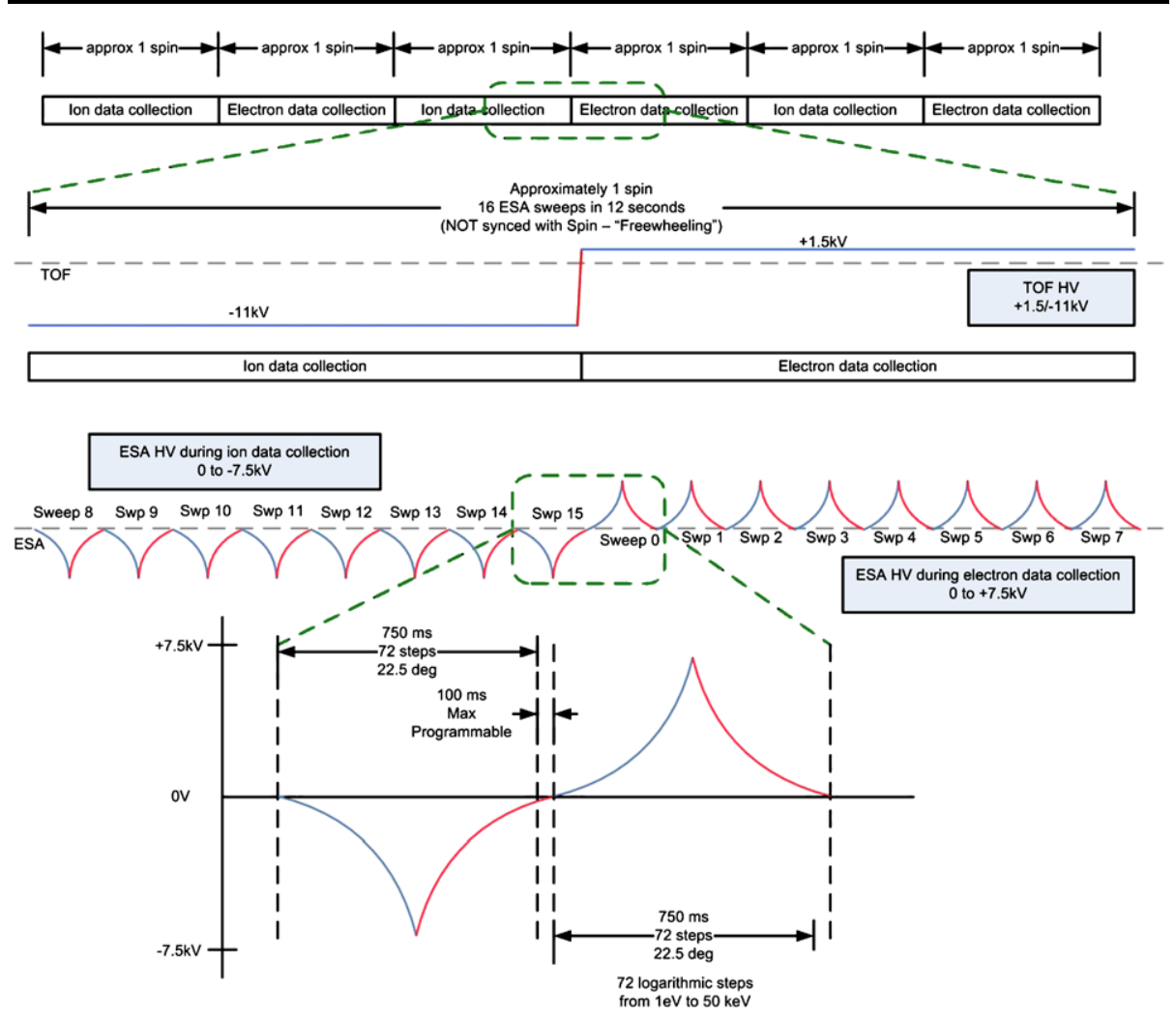

Fig. 22 Timing diagram for a series of HOPE spins and ESA sweeps. Electron and ion frames are acquired over alternating sets of 16 full ESA sweeps. A Master Frame is composed of one electron and one ion frame and is acquired over $24 \mathrm{sec}$ based on $\tau_{\text {ESA_STEP }}=10.417 \mathrm{~ms}$

the taps sequentially register a high state in the FPGA. At each leading edge of the $40 \mathrm{MHz}$ clock pulse, the TOF FPGA polls its tap inputs for high states. If one or more high states are present, the number $N_{\mathrm{Hi}}$ of high input states is counted and reported. The fine resolution time measurement is simply $\tau_{\mathrm{FINE}}=2.5 \mathrm{~ns} \times N_{\mathrm{Hi}}$.

Figure 23 (bottom panel) illustrates the measurement of an ion whose TOF in the Drift Box is $\tau_{\mathrm{TOF}}=95 \mathrm{~ns}$. The Start pulse enables coarse counting of the $40 \mathrm{MHz}$ clock pulses. It additionally registers high tap states in the FPGA, which are then counted at the leading edge of the first clock pulse; the six high states at $2.5 \mathrm{~ns}$ each yields $\tau_{\text {FINE_ST }}=15 \mathrm{~ns}$. The coarse counting $40 \mathrm{MHz}$ clock pulses continues until a Stop pulse is registered, and the number of counted clock pulses $(4 \times 25 \mathrm{~ns})$ yields $\tau_{\mathrm{COARSE}}=100 \mathrm{~ns}$. Finally, the number of high states $(4 \times 2.5 \mathrm{~ns})$ in the Stop taps at the last rising edge of the clock is counted, providing $\tau_{\text {FINE_ST }}=10 \mathrm{~ns}$. The ion's TOF, including the initial $10 \mathrm{~ns}$ time delay applied to Stop signals, is therefore $\tau_{\mathrm{TOF}}=95 \mathrm{~ns}$.

An event is registered when a start pulse and/or a stop pulse is registered. Eight event counters are used to track all events at each energy step and each of the five polar pixels. The logical sequences that increment each counter are illustrated in Fig. 24. Every Start and Stop count increments the Start and Stop counters, respectively. Additionally, an event is classified by one of six possible outcomes: valid $\tau_{\mathrm{TOF}}$ events falling within the $\mathrm{H}^{+}, \mathrm{He}^{+}$, and $\mathrm{O}^{+}$TOF windows; invalid $\tau_{\mathrm{TOF}}$ events in which a Stop is recorded within $160 \mathrm{~ns}$ of the Start 


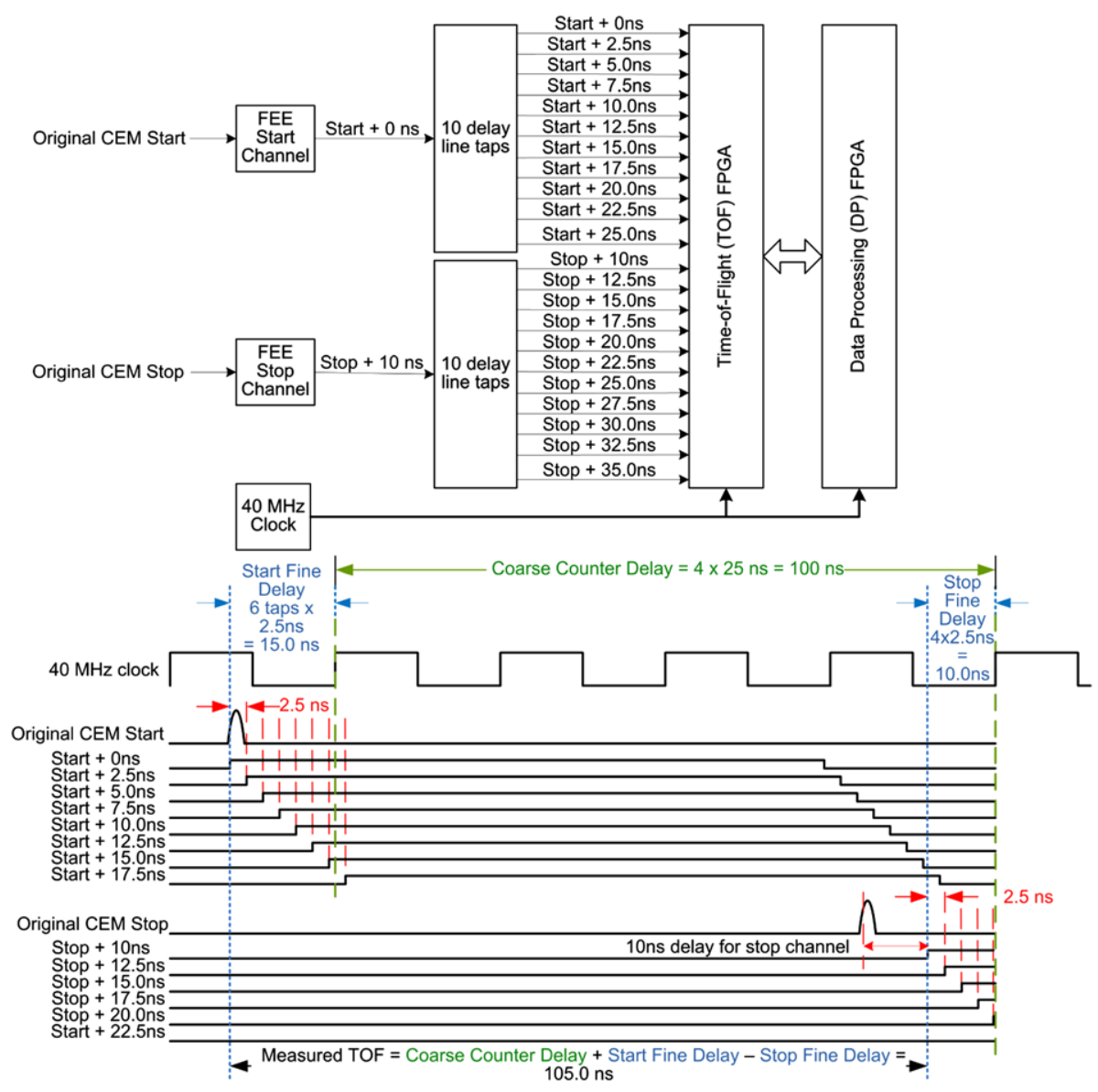

Fig. 23 Block diagram and timing diagram illustrating the time-of-flight calculation algorithm in the TOF FPGA for an ion with a measured $115 \mathrm{~ns}$ TOF. The 4 coarse counts of $40 \mathrm{MHz}$ clock periods (25 ns each) and the fine measurements of six and four $2.5 \mathrm{~ns}$ intervals registered for the Start and Stop combine to result in a measured TOF of $105 \mathrm{~ns}$. A $10 \mathrm{~ns}$ delay is added to the Stop signal before it is input into the TOF FPGA to ensure that the Stop signal is registered after the Start signal; the actual particle TOF in the Drift Box is therefore $95 \mathrm{~ns}$

but does not fall within the $\mathrm{H}^{+}, \mathrm{He}^{+}$, and $\mathrm{O}^{+}$TOF windows; and Starts registered without a Stop within the following $160 \mathrm{~ns}$; and Stops with no Starts registered within the preceding $160 \mathrm{~ns}$. The TOF window for each species is set by the TOF LUT for each energy step. Because $\tau_{\mathrm{TOF}}$ of incident electrons is short and no other species are present, the classification scheme in electron mode is simpler; electron TOF is short, and all longer TOF events within $160 \mathrm{~ns}$ are retained as a measure of penetrating background. This scheme carries redundancy that can be used to check and validate the event electronics, if needed; specifically, the number of events from any three of the four event counters Start, Stop, Start-no-Stop, and Stop-no-Start can be used to calculate what should be observed in the fourth counter.

The range of expected TOF values for valid particles is $0-160 \mathrm{~ns}$ based on the length of the drift region and range of ion energies that HOPE will measure. The species is determined from a configurable lookup table (LUT) which outputs the calculated species based 


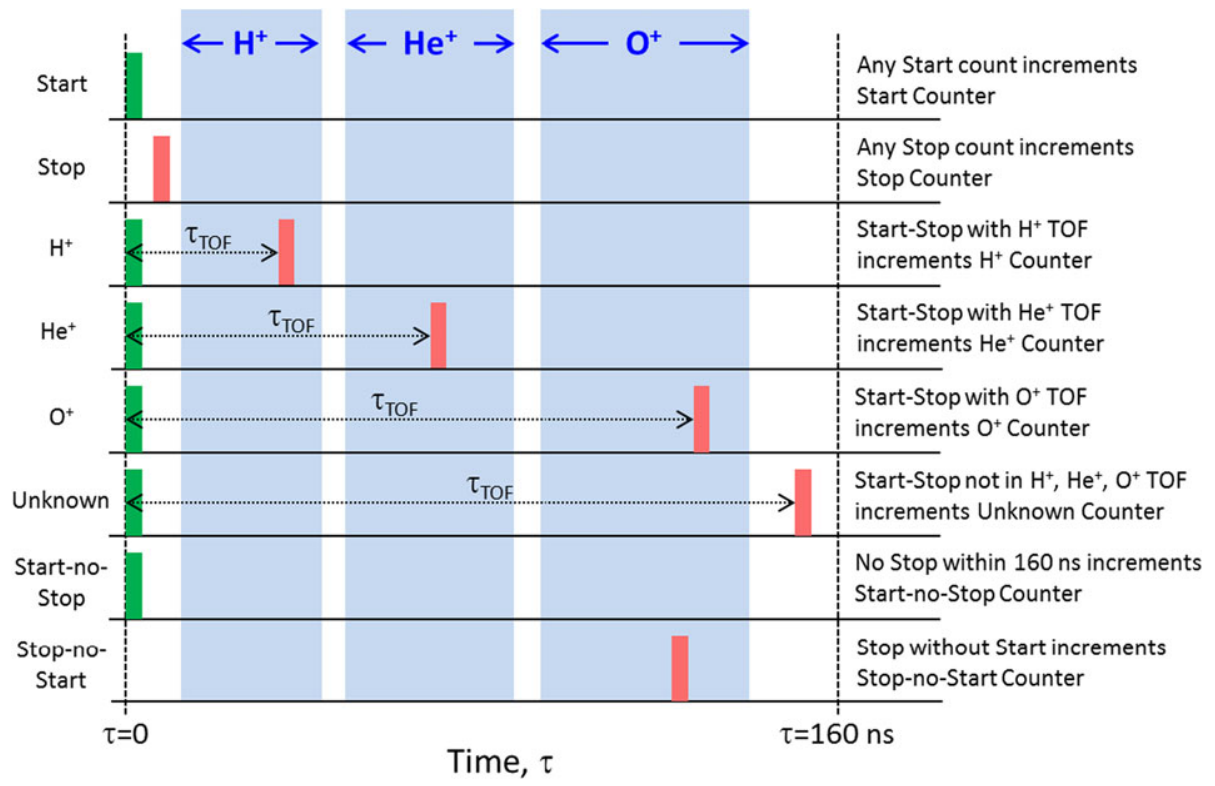

Fig. 24 HOPE uses eight types of counters for classification of events at each energy step for each polar pixel. Ion mode classification is shown. Every event increments either (or both) of the Start and Stop Counters and also increments one of six counters based on classification of the event as shown. The blue time-of-flight regions for $\mathrm{H}^{+}, \mathrm{He}^{+}$, and $\mathrm{O}^{+}$are unique for each energy step and are defined in a LUT

on the current energy step and the TOF value for a valid particle. For each of the five pixels, the TOF FPGA is able to account for all particles up to a $2 \mathrm{MHz}$ constant periodic rate. After every ESA step, the TOF sends its counter information and two histograms to the DP FPGA. The DP FPGA stores the raw data into $2 \times 512 \mathrm{kB}$ ping-pong science memories, coordinated with ESA sweeping and ESA and TOF polarity changes that toggle between ion and electron modes.

The DP FPGA is capable of performing summing ("collapse") operations to stay within telemetry limits while retaining all acquired counts. For nominal science mode, adjacent ESA steps are summed so that the number of reported steps is reduced from 72 to 36 . Independent of energy collapse, the DP FPGA can also collapse the reported azimuthal angles to reduce that dimension by factors of $2,4,8$ or 16 . In the nominal HOPE azimuth collapse scheme, schematically illustrated later in Fig. 35, polar pixels 1 and $5\left( \pm 72^{\circ}\right.$ polar angles) are each collapsed into 4 azimuthal bins, polar pixels 2 and $4\left( \pm 36^{\circ}\right)$ are each collapsed into 8 azimuthal bins, and polar pixel $3\left(0^{\circ}\right)$ is not collapsed, retaining all 16 azimuthal bins. After collapsing, data compression from a 16-bit to 8-bit number can be performed via reconfigurable lookup table. The DP FPGA performs the collapse and compression processes "on-the-fly".

\subsubsection{HOPE Operational Modes}

The HEU controls the operational mode of HOPE as dictated by spacecraft commands, including the acquisition, processing and telemetering of science data to the spacecraft. The HEU reports health and safety data via engineering telemetry and performs autonomous saving actions. It also controls autonomous recovery from anticipated events such as thruster 
Fig. 25 HOPE mode transition diagram

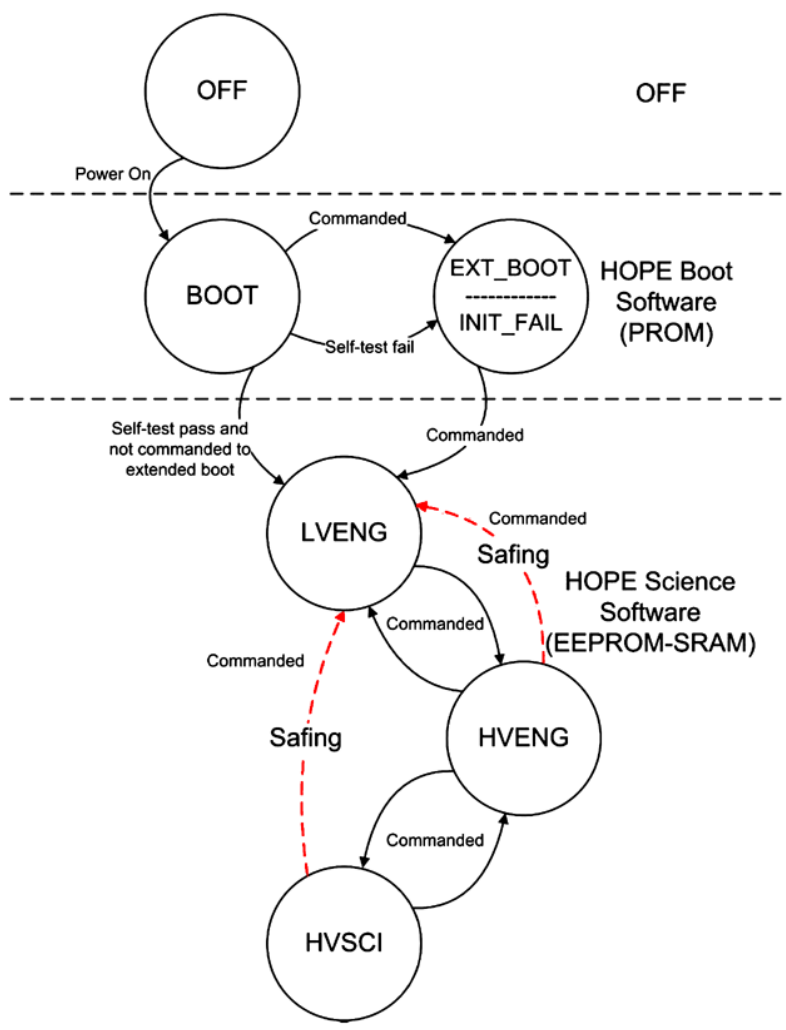

firing so as to maintain high availability for science acquisition with minimal ground interaction. Timestamping of the science and engineering telemetry is synchronized with the 1-PPS and timestamp messages from the spacecraft. As the mission progresses, the HEU can be updated via configured changes to onboard flight software and lookup tables.

HOPE has four distinct modes of operation, whose relationships are shown in Fig. 25: the bootup phase (called BOOT) after initial power on; low-voltage engineering (LVENG), high-voltage engineering (HVENG) and high-voltage science (HVSCI). During nominal science operation (HVSCI), the HEU sets the bias $V_{\mathrm{CEM} \_\mathrm{R}}$ of the CEMs at predetermined levels and controls the sweeping of the ESA and TOF during science acquisition. This activity is coordinated with the collection of the CEM output signals by the FEE, which converts those signals into digital pulses for the DB. The DB calculates the time-of-flight (TOF) between each pair of a start-stop CEM pixel set and organizes the data as various raw histograms, stored as a function of energy step and polar pixel. These raw histograms consist of eight event counters as described in Fig. 24. The flight software, which resides in the DB, coordinates the output of the science data via telemetry by instructing the DB to create a telemetry stream from the raw histograms by specifying a subset to be output. The amount of collapsing (i.e., summing of adjacent energy and azimuthal angle indices in the histogram) and 16-bit to 8-bit compression (via lookup table) are also specified.

Because different operational modes employ different combinations of circuits with different load levels, HOPE power consumption varies, with Boot and LVENG modes using 10.2 W, HVENG at $11.1 \mathrm{~W}$, and HVSCI using $18.4 \mathrm{~W}$. 


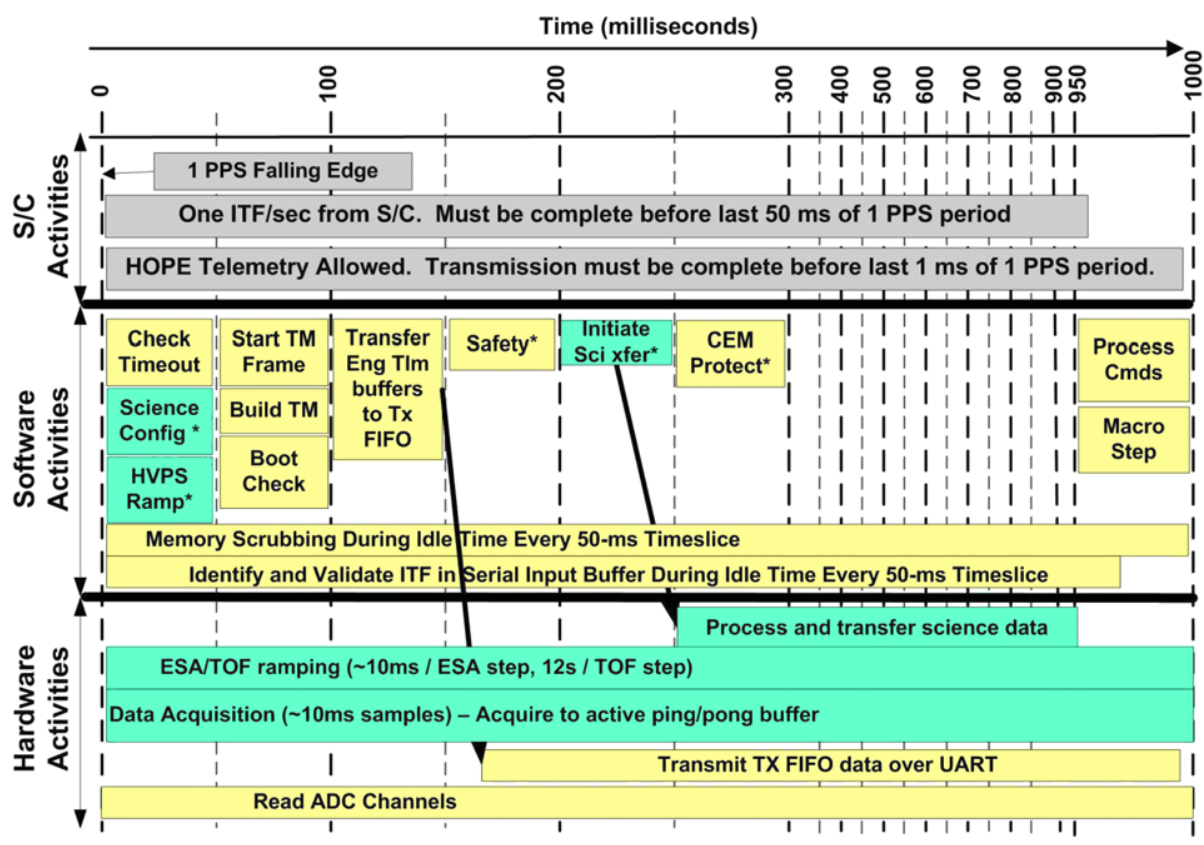

Fig. 26 One-second time-slice used for scheduling HOPE FSW tasks

\subsubsection{Flight Software}

The HOPE Flight Software (FSW) keeps track of the instrument mode; manages command, telemetry and synchronization interfaces with the $\mathrm{S} / \mathrm{C}$; manages science acquisition; maintains instrument integrity through scrubbing the memory and tracking health and safety; and executes recovery operations when applicable. The FSW runs on the Actel 8051 core that resides in the SI FPGA and is written in the C programming language. The code has a time-slice architecture which uses the 1-PPS signal from the spacecraft to synchronize its task timeline with an external reference. FSW tasks are assigned to one of twenty 50-ms time slices within the one-second period. An illustration is shown in Fig. 26.

The HOPE instrument is commanded via CCSDS command packets received through the S/C LVDS command interface.

To maintain configurability without needing to upload new code during ground and flight operations, the FSW manages a lookup table stored within HOPE in non-volatile EEPROM memory which has onboard parameter groups as shown in Table 5.

Data is collected and sent to the spacecraft during HVSCI operations using science acquisition plans and telemetry definitions. These settings include the ESA sweep table, the polarity (ions or electron mode), algorithm for collapse in energy or angle, selection of event counters sent for telemetry, and whether the EFW or EMFISIS burst flags are enabled. When a burst flag is detected and its corresponding burst flag is enabled, a different entry within the science acquisition plan table is chosen to run for a predetermined amount of time from a LUT entry; after this time expires, the operations revert to nominal HVSCI. The HOPE flight software and LUTs are sufficiently flexible that plans can be encoded to control HOPE to execute all currently envisioned HVSCI science acquisition and telemetry scenarios. 
Table 5 List of HOPE LUT parameter groups

\begin{tabular}{|c|c|}
\hline LUT parameter group & Description \\
\hline Science acquisition plans & $\begin{array}{l}\text { A series of steps executed during HVSCI defining data collection } \\
\text { parameters and telemetry products output to the spacecraft }\end{array}$ \\
\hline $\begin{array}{l}\text { ESA and TOF high-voltage sweep } \\
\text { tables }\end{array}$ & $\begin{array}{l}\text { LUTs containing the DAC settings for the ESA and TOF HVPS } \\
\text { during HVSCI sweep }\end{array}$ \\
\hline $\begin{array}{l}\text { Space weather telemetry } \\
\text { configuration }\end{array}$ & $\begin{array}{l}\text { Instructions specifying what information (e.g., species, energies) is } \\
\text { placed into the Space Weather telemetry packet }\end{array}$ \\
\hline Energy-TOF mapping to species & $\begin{array}{l}\text { LUT for each polar pixel that associates a measured TOF with an ion } \\
\text { species at each energy step. }\end{array}$ \\
\hline TOF histogram sampling control & $\begin{array}{l}\text { Table that controls the telemetry output of the raw TOF histogram } \\
\text { data after all event counters have been telemetered }\end{array}$ \\
\hline Data compression table & LUT used by the DB to convert 16-bit values to 8-bit values \\
\hline Macros & A series of scripts containing sequenced HOPE commands \\
\hline CEM protection levels & $\begin{array}{l}\text { LUT of reduced CEM voltage levels after a thruster warning has } \\
\text { been issued by the S/C }\end{array}$ \\
\hline Engineering safety & $\begin{array}{l}\text { LUT of all onboard engineering parameters and their red levels that } \\
\text { can autosafe HOPE }\end{array}$ \\
\hline Engineering configurable settings & Default engineering values at power-on \\
\hline
\end{tabular}

\section{HOPE Performance}

\subsection{Performance Validation: Calibration}

End-to-end HOPE performance is derived through a combination of component-level and subsystem-level tests, modeling and simulation that are subsequently empirically validated, and instrument calibration. Although calibration provides the most accurate description of instrument performance, it cannot provide a full picture of the instrument for two reasons. First, the parameter space covered by HOPE is large: 5 polar pixels, 72 energy steps, four species $\left(\mathrm{e}^{-}, \mathrm{H}^{+}, \mathrm{He}^{+}, \mathrm{O}^{+}\right)$, and variable response in both polar and azimuth angles. This complexity precludes measurement of each unique parameter combination. In addition, determination of the instrument geometric factor for space plasmas, which uniformly fill a pixel in azimuth angle, polar angle, and energy, is not readily obtained from the monoenergetic, highly collimated ion beams available in the laboratory. Because of these limitations, laboratory measurements are used to validate the electron-optic model of the ESA subsystem, which is then combined with the sensitivity of the TOF subsystem derived from calibration. The instrument response over the full range of input particles (species, energy, polar angle, azimuth angle) is derived by interpolation and extrapolation of the combination of the ESA and TOF subsystem performances.

HOPE calibration was performed in three phases: prior to instrument environmental testing (Phase 1), after instrument-level environmental testing (Phase 2), and after detector refurbishment and flight HVPS installation that followed spacecraft-level testing (Phase 3). Engineering model HVPS were used for Phases 1 and 2; for spacecraft level testing between Phases 2 and 3, the flight detector assemblies were replaced with engineering model assemblies to prevent particulate, hydrocarbon, and water vapor contamination of the flight CEM detectors. Calibration was performed at the Los Alamos calibration facility using ion and electron beams, described in the Appendix. Because of its beam stability over time and 
its central location in the HOPE energy range, a $10 \mathrm{keV} \mathrm{H}^{+}$beam was selected as the primary calibration fiducial energy and species for testing across all pixels, both instruments, and all calibration phases.

\subsubsection{Energy and Angle Response}

HOPE energy, polar angle, and azimuth angle responses are characterized using a monoenergetic beam at energy $E_{0}$ of electrons or mass-selected ions $\left(\mathrm{H}^{+}, \mathrm{He}^{+}\right.$, and $\left.\mathrm{O}^{+}\right)$. Because the polar angle $\theta$ follows the cylindrical symmetry of the ESA subsystem, the energy response is generally independent of polar angle. However, the energy and azimuth angle responses are strongly coupled, and we measure this coupled response by rotating the instrument through azimuth angle $\phi$ and scanning $V_{\mathrm{ESA}}$. High-resolution scans were performed at azimuthal steps of $0.7^{\circ}$ and using $V_{\mathrm{ESA}}$ steps of $\sim 20 \mathrm{~V}$; scans at lower resolution were used to verify performance, e.g., during Phase 3 calibration. From these scans we derive the electrostatic analyzer $k$ factor $\left(k=E_{0} / e V_{\mathrm{ESA}}\right)$, energy resolution, azimuth angle resolution, and the coupled energy-azimuth angle response. Results are compared with SIMION model results to validate the electron-optic model used for calculation of the ESA subsystem geometric factor $G_{\mathrm{ESA}}$.

These scans were performed using $10 \mathrm{keV} \mathrm{H}^{+}$for each polar pixel. In addition, measurements were made for $5 \mathrm{keV} \mathrm{H}^{+}, \mathrm{He}^{+}$, and $\mathrm{O}^{+} ; 30 \mathrm{keV} \mathrm{H}^{+}$and $\mathrm{O}^{+}$; and $45 \mathrm{keV} \mathrm{H}^{+}$, $\mathrm{He}^{+}$, and $\mathrm{O}^{+}$for a single polar pixel for each instrument. Electron scans were performed at $10 \mathrm{keV}$ for three HOPE-A polar pixels, and at 5, 10, and $20 \mathrm{keV}$ for a single HOPE-B polar pixel.

Figure 27 shows data from the high-resolution scans for HOPE-A and HOPE-B. The ion results are nearly identical to electron results, which are not shown. For each instrument the middle panel shows counts as a function of azimuthal angle $\phi$ and $V_{\mathrm{ESA}}$. These plots clearly show the coupled energy-angle response characteristic of a top-hat electrostatic analyzer. The left and right panels show these data collapsed as a function of $V_{\mathrm{ESA}}$ and $\phi$, respectively, and are used to determine the peak location and peak width of the response in both $V_{\mathrm{ESA}}$ and $\phi$. These panels also show Gaussian fits to the collapsed data, which are used to quantify the response as a function of $V_{\mathrm{ESA}}$ and $\phi$.

The results of the energy-angle scans are consistent throughout all calibration phases and across all polar pixels of both instruments. Based on average results, with extra weight given to the high-resolution scans, the calibration data yield $k=7.035$ for both HOPE-A and HOPE-B. For comparison, SIMION simulations of the flight geometry yielded $k=6.9$. The $<2 \%$ difference between calibration and SIMION simulations is consistent with tolerance stack-up in fabrication and assembly of the ESA subsystem, which was measured during flight assembly for both A and B flight units.

Polar angle $(\theta)$ scans were performed using a mono-energetic ion or electron beam at a fixed azimuth angle. Figure 28 shows high-resolution polar scan results from HOPE-A and HOPE-B, respectively, for $10 \mathrm{keV} \mathrm{H}^{+}$incident at azimuth angle $\phi=0^{\circ}$. Measurements were made at polar angle steps of $1^{\circ}$, and ion beam drift was monitored by periodically repeating the measurement at the center of Pixel $3\left(\theta=0^{\circ}\right)$. Each panel shows data from both total Starts (black) and valid Coincidence counts (red).

These results show consistency of absolute response across all five polar pixels, as well as no overlap in the polar response between these pixels. Systematic pixel-to-pixel variations are observed at a level $<5 \%$. The peak response of each polar pixel is within $1^{\circ}$ of the locations $\pm 72^{\circ}, \pm 36^{\circ}$, and $0^{\circ}$ predicted by charged particle optics modeling. HOPE-A polar pixels $1-5$ were observed to have a maximum response for coincidences at polar angles 

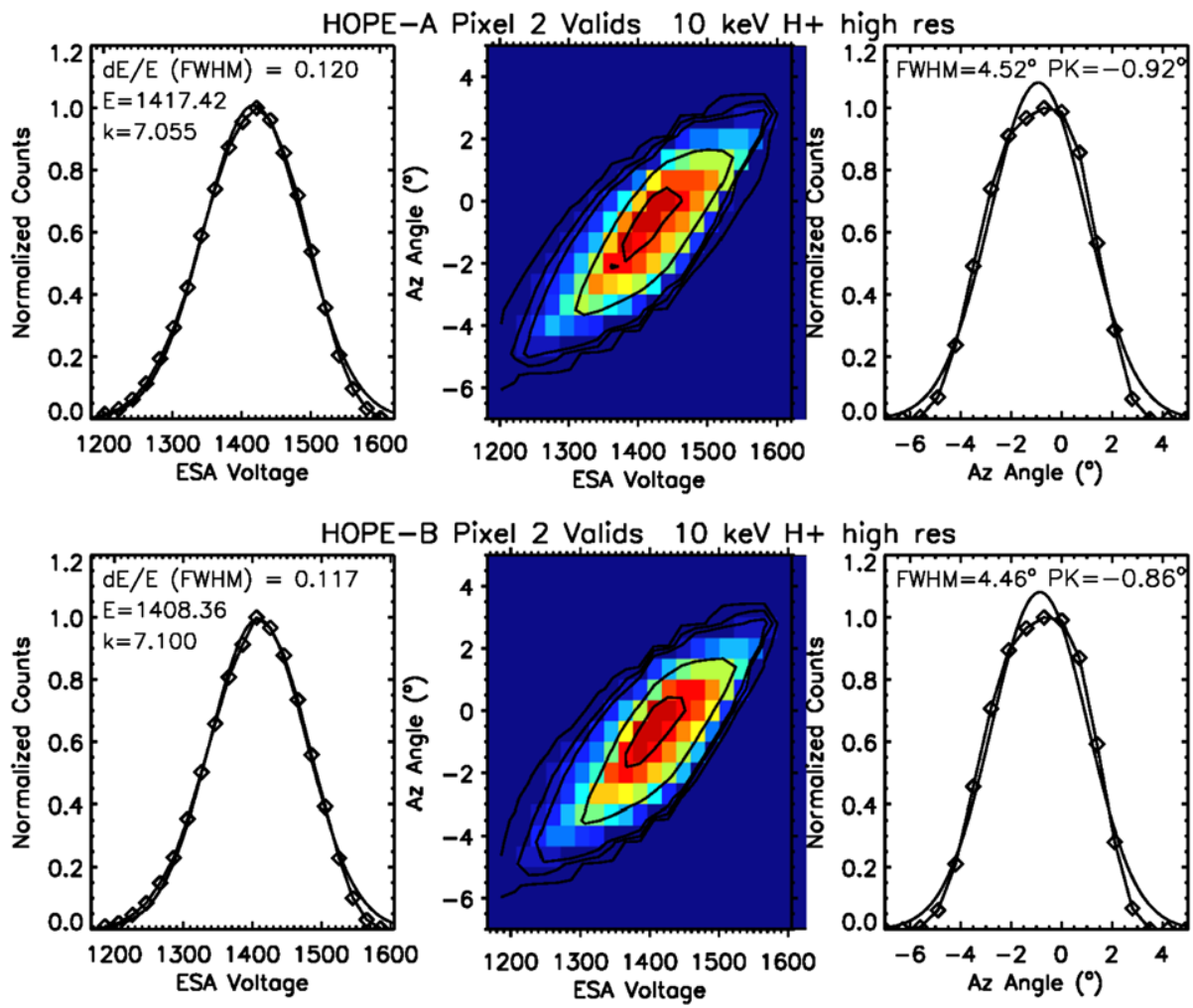

Fig. 27 HOPE-A (top panels) and HOPE-B (bottom panels) energy-azimuthal angle responses for valid coincidence events from the high-resolution energy-angle scan for incident $10 \mathrm{keV} \mathrm{H}^{+}$

$+71^{\circ},+36^{\circ}, 0^{\circ},-35^{\circ}$, and $-72^{\circ}$; HOPE-B peak maxima were observed at polar angles $+72^{\circ},+36^{\circ}, 0^{\circ},-36^{\circ}$, and $-71^{\circ}$.

Precision alignment measurements were made for both flight instruments using a theodolite. One reference point was the ion beam, highly collimated by upstream apertures in the calibration facility. The second point was a mirror affixed to an external surface of HOPE and referenced to the HOPE-spacecraft mating surface. The instrument was oriented in the calibration chamber at the location of maximum response to the ion beam, and the orientation of the instrument relative to the ion beam was precisely measured; the resulting alignment offset of HOPE-A was $(\phi, \theta)=\left(-0.57^{\circ},-0.06^{\circ}\right)$ and of HOPE-B was $\left(-1.22^{\circ},-0.15^{\circ}\right)$.

\subsubsection{Detector Gain}

The CEM gain is set such that secondary electrons generated by incident ions or electrons in the TOF subsystem yield a pulse magnitude in the CEM that is larger than the lower level discriminator setting of the amplifier electronics. This level is attained by increasing the CEM bias until the count rate of valid events becomes approximately constant, independent of further increase in CEM bias. However, as a CEM ages, its gain decreases at a rate that primarily depends on the total charge output by the CEM over its lifetime. This gain decrease is typically compensated by periodically increasing the bias applied to the CEM. Regular gain calibration tests are scheduled throughout the RBSP mission. This is performed at a 
Fig. 28 HOPE-A (top panel) and HOPE-B (bottom panel) high-resolution polar angle $(\theta)$ scans across all five pixels in each instrument for incident $10 \mathrm{keV} \mathrm{H}^{+}$. In each plot, the Start count rate is shown in black and the Coincidence rate in red
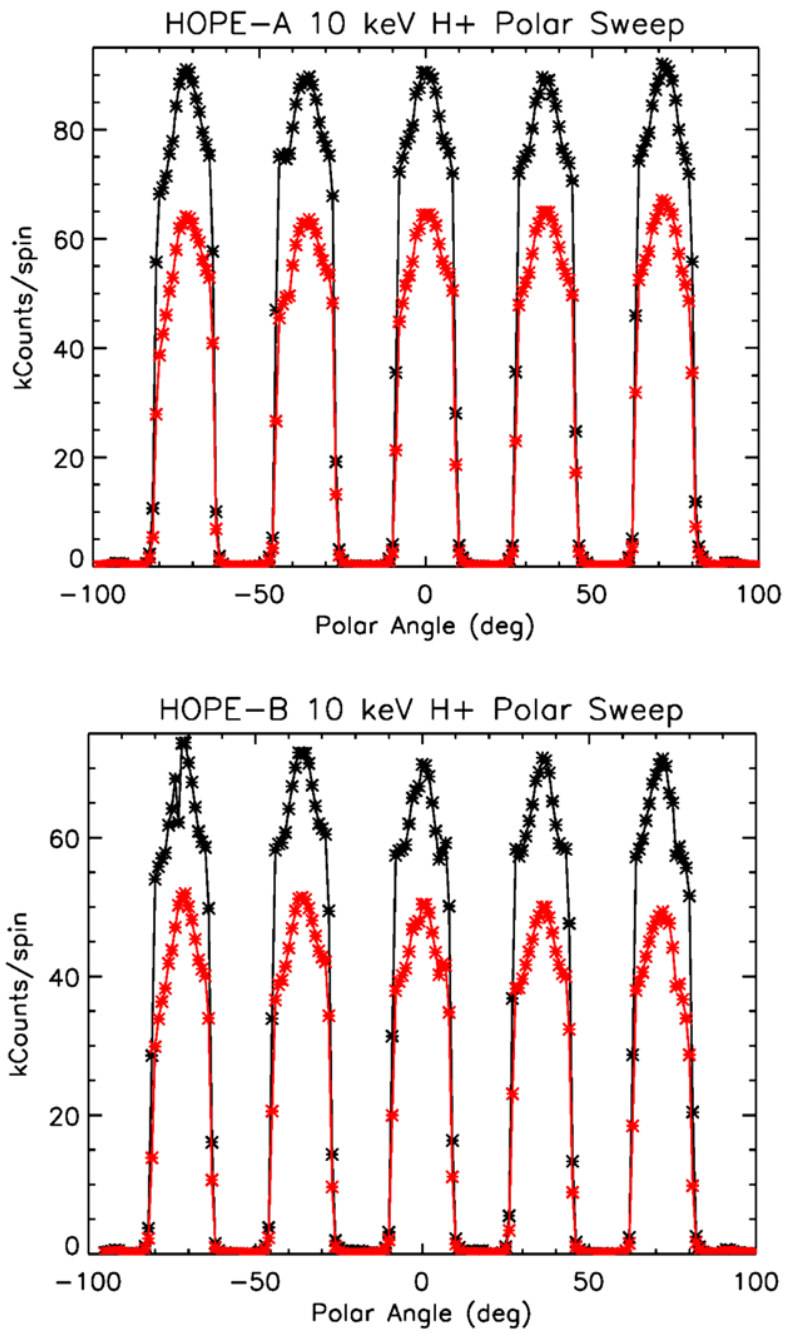

fixed ESA voltage level in ion and electron modes, with data collected over a range of CEM voltage levels.

Because CEMs can age differently, HOPE utilizes three different CEM bias supplies that service polar Pixels 1 and 4, Pixels 2 and 5, and Pixel 3. While the fronts of the CEMs are fixed at $V_{\text {CEM_F }}=+4.4 \mathrm{kV}$, the adjustable voltage $V_{\text {CEM_R }}$ applied to the rear of the CEMs can be changed based on the gain calibration tests. The nominal voltage for HVSCI operation at commissioning is $V_{\text {CEM R }}=+6.8 \mathrm{kV}$. At this voltage, the lower level discriminator (LLD) is set at a value of 100 to retain nearly all real CEM pulses and exclude noise counts of lower pulse magnitudes. The LLDs have a large dynamic range (0-4095) to accommodate variations in CEM gain expected through the RBSP mission; we note that during calibration an LLD setting of 2500 eliminated all CEM pulses from being counted.

The CEMs were originally selected and matched as polar pixels based on similar gain curves and similar intrinsic impedances. The gain curves for each of the CEMs in the flight instruments acquired during Phase 3 Calibration are shown in Fig. 29. 
Fig. 29 Gain curves for HOPE A (top) and HOPE B (bottom). Start counts are shown with dashed lines and " $x$ " symbols, stop counts with solid lines and diamond " $\diamond "$ symbols. Each curve is normalized to a value of 1.0 at $V_{\text {CEM_R }}=6.90 \mathrm{kV}$
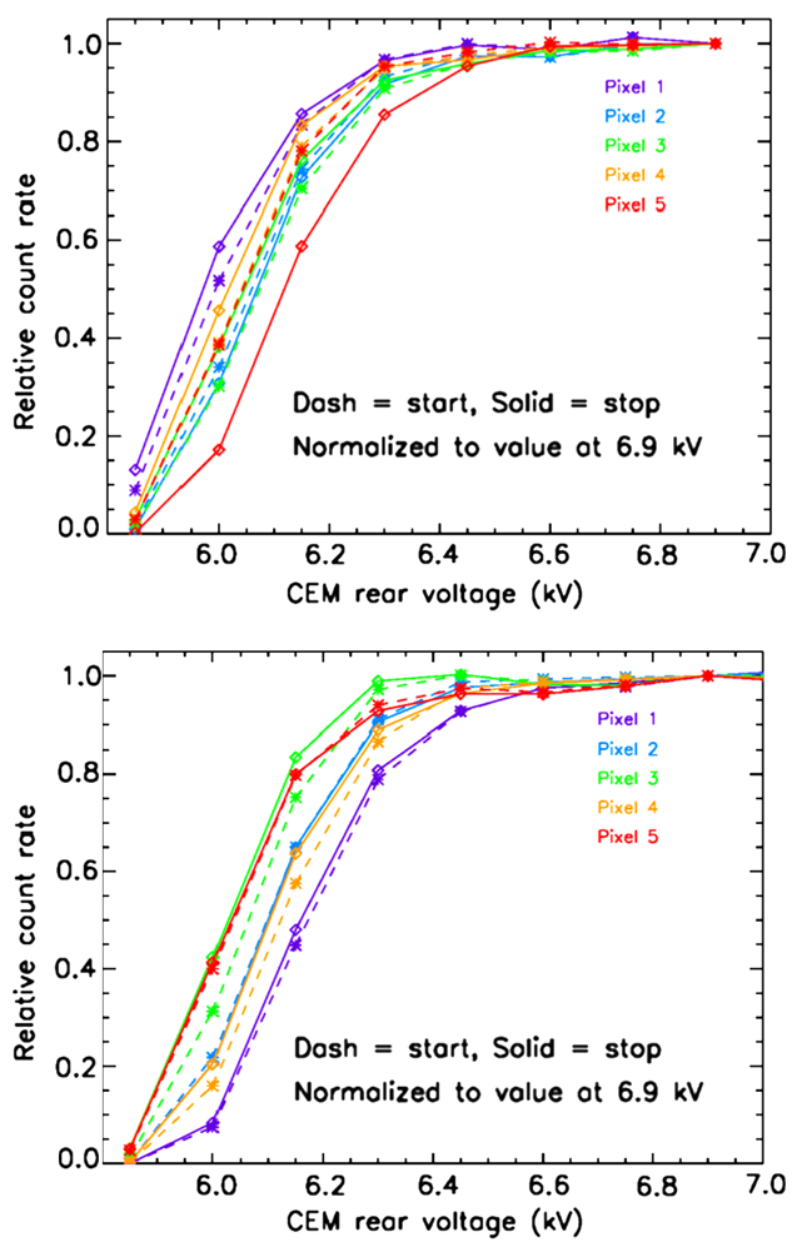

\subsubsection{Sensitivity}

Knowledge of the absolute coincidence detection efficiencies for each polar pixel is critical for deriving accurate distribution functions. In spite of the complexity of the TOF subsystem (including the electron-optics and the physical processes that govern secondary electron emission, the trajectories of incident particles in the TOF drift box, and individual CEM detector efficiencies), we can calculate the absolute detector and coincidence detection efficiencies for each polar pixel simply using the total Coincidence, Start and Stop counts over some time interval (Funsten et al. 2005). These values have been measured throughout calibration and will be monitored throughout the mission for each CEM detector. This technique was first incorporated on the IBEX-Hi energetic neutral atom imager (Funsten et al. 2009) and has been an important tool for quantifying, identifying, and monitoring different types of background in IBEX-Hi.

For each polar pixel, HOPE reports the number of total (coincident and non-coincident) Start counts $C_{\mathrm{ST}}$, total Stop counts $C_{\mathrm{SP}}$, and coincidence counts $C_{\mathrm{COIN}}$ over a particular time interval. The absolute Start and Stop detection efficiencies are $\varepsilon_{\mathrm{ST}}=C_{\mathrm{COIN}} / C_{\mathrm{SP}}$ and $\varepsilon_{\mathrm{SP}}=$ $C_{\mathrm{COIN}} / C_{\mathrm{ST}}$, respectively. The instrument response is directly proportional to the absolute 


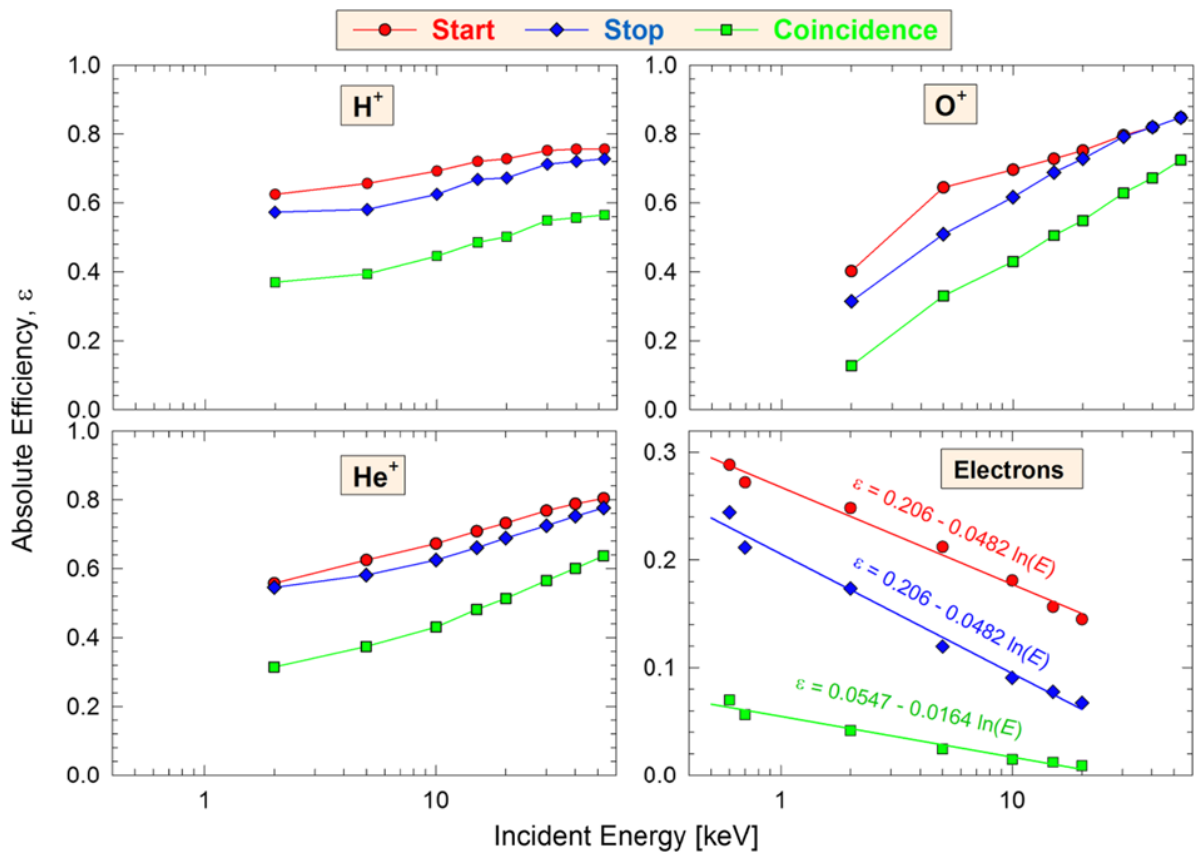

Fig. 30 Typical absolute detection efficiencies for incident ions (Polar pixel 3 of HOPE B) and electrons (Polar pixel 5 of HOPE-A). The Coincidence efficiency is the product of the Start and Stop efficiencies. Detector singles (non-coincident) counts and coincident counts are reported for all detectors throughout the mission, enabling monitoring of TOF subsystem performance and identification and quantification of background from penetrating radiation

coincidence efficiency $\varepsilon_{\mathrm{COIN}}=\varepsilon_{\mathrm{ST}} \varepsilon_{\mathrm{SP}}$, which will likely change as the CEM detectors age during the mission. Typical efficiencies for HOPE are shown in Fig. 30. During times of low penetrating background, $\varepsilon_{\mathrm{ST}}, \varepsilon_{\mathrm{SP}}$, and $\varepsilon_{\mathrm{COIN}}$ are characteristic of the targeted ions or electrons being measured. In ion mode, the relative abundances of the ion species must be considered because the ion species have different detection efficiencies. To measure and monitor the absolute detection efficiency during the mission, we will target times of high $\mathrm{H}^{+}$abundance with low background count rates.

Penetrating background generates mostly non-coincident Starts and Stops, and a coincidence generated by a single penetrating particle will have a short TOF, similar to plasma electrons and fast protons. We therefore use Start and Stop singles rates and Coincidence rates in ion mode to monitor penetrating radiation in general and use long TOF measurements in electron mode as a consistency measurement of random coincidence from events generated by uncorrelated penetrating particles.

Figure 31 shows the measured absolute coincidence efficiencies derived for each polar pixel of HOPE A and B flight units both after environmental testing and after refurbishment of the CEM detectors. A polynomial fit to the measured values was derived for calculation of the absolute HOPE geometric factor. As the incident particle energy approaches $1 \mathrm{eV}$, the efficiency approaches a constant value because of acceleration into the TOF subsystem by $V_{\mathrm{TOF}}=-11 \mathrm{kV}$ for ions and $V_{\mathrm{TOF}}=+1.5 \mathrm{kV}$ for electrons. The data show consistent results at the two different calibration times across all pixels and between the HOPE A and 


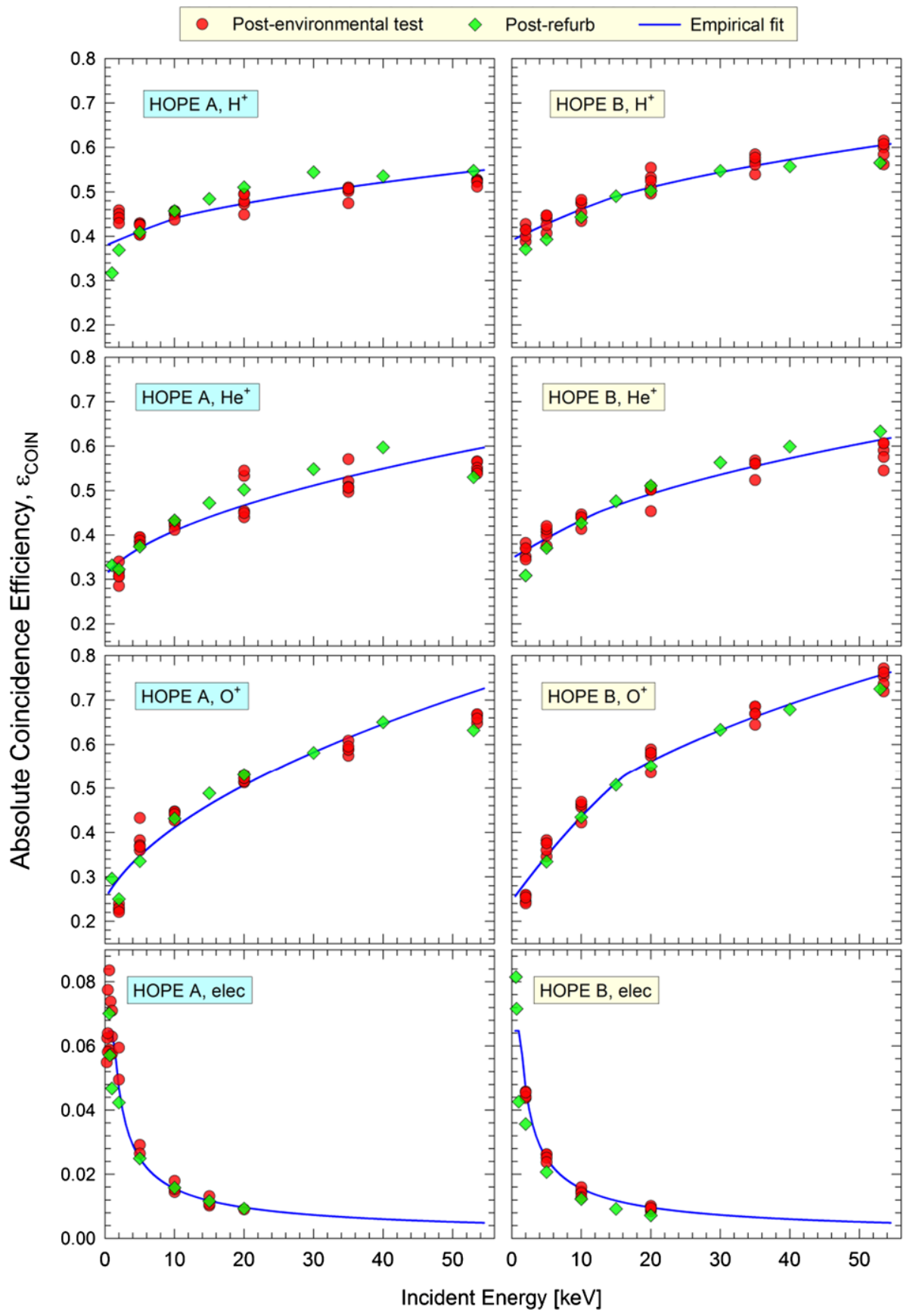

Fig. 31 Absolute coincidence detection efficiencies incident ions (top three panels) and electrons (bottom panels) for all pixels of HOPE A (right panels) and HOPE B (left panels). The red points are measurements acquired after environmental testing and the green points are derived from calibration after CEM detector refurbishment. The blue lines are empirical polynomial fits that are used in the calculation of the total HOPE Geometric factor 
Fig. 32 HOPE geometric factor for each polar pixel is shown for ions (top panel) and electrons (bottom panel)

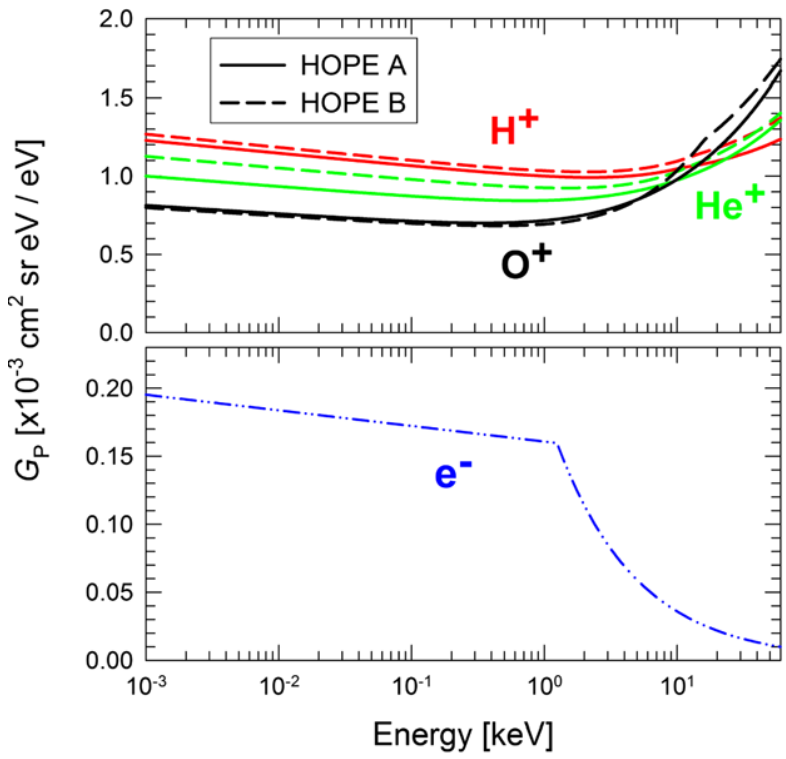

B flight units. The electron efficiencies of HOPE A and B were sufficiently similar so that a single polynomial function was used to describe the efficiencies for both instruments.

The HOPE geometric factor per pixel, which is the cumulative instrument response for conversion of instrument counts to ion or electron flux, is derived using

$$
G_{\mathrm{P}}=\varepsilon_{\mathrm{COIN}} G_{\mathrm{ESA}} T_{\mathrm{G}}
$$

where $T_{\mathrm{G}}=0.7$ is the geometrical transmission of the grid on which the Start foil is attached, $\varepsilon_{\mathrm{COIN}}$ is obtained from Fig. 31, and $G_{\mathrm{ESA}}$ is obtained from Fig. 11. The resulting HOPE A and B geometric factors are shown in Fig. 32 for ions and electrons. Slight variations are observed between A and B flight units.

\subsubsection{Species Identification}

HOPE identifies species through time-of-flight measurement of species that have been filtered based on energy-per-charge in the ESA Subsystem. As previously described, HOPE represents the first use of CEM detectors for TOF mass spectrometry in space, and the mass identification performance of HOPE is an important validation of this technique. The HOPE mass resolution is a function of three processes: the energy resolution of the ESA subsystem; energy and angular straggling of ions in the foil; and the fundamental, energy-independent time dispersion $\delta \tau_{\mathrm{TOF}}$ of the TOF subsystem that includes secondary electron transport to the detectors, the temporal response of the CEM detectors, and the TOF electronics. We first present calibration data for a monoenergetic ion beam, then quantification of $\delta \tau_{\mathrm{TOF}}$ using commissioning data, and finally TOF spectra from commissioning.

The governing equation for the HOPE mass spectrum for an ion with energy $E$ at the HOPE aperture entrance is:

$$
m[\mathrm{amu}]=2 u\left(\frac{\tau_{\mathrm{TOF}}[\mathrm{ns}]-10 \mathrm{~ns}}{d[\mathrm{~cm}]}\right)^{2}\left(E[\mathrm{keV}]+\left|e V_{\mathrm{TOF}}[\mathrm{kV}]\right|-E_{\mathrm{F}}[\mathrm{keV}]\right)
$$


where $\tau_{\mathrm{TOF}}$ is the measured particle TOF, $10 \mathrm{~ns}$ corresponds to the delay line incorporated into the Stop electronics, $d$ is the pathlength of the particle in the TOF box $(\sim 3 \mathrm{~cm}), e=1$ is the electron charge, and the constant $u=9.58 \times 10^{-4}$ represents all unit conversions. The ion energy at the exit surface of the foil is the sum of its initial energy and additional acceleration by the bias of the TOF, less the energy loss $E_{\mathrm{F}}$ in the foil. Because the ion speeds are generally less than the Bohr velocity, we approximate a velocity-proportional energy loss in the foil (Lindhard et al. 1963) using

$$
E_{\mathrm{F}}=k\left(E+\left|V_{\mathrm{TOF}}[\mathrm{kV}]\right|\right)^{1 / 2}
$$

which has been shown to reasonably represent energy loss in a foil (Allegrini et al. 2006).

In practice, $m$ is not a discrete function, rather a continuous function driven by uncertainties or dispersion in $\tau_{\mathrm{TOF}}, E, d$, and $E_{\mathrm{F}}$. Figure 33 shows HOPE mass spectra $m$ for incident ${ }^{1} \mathrm{H}^{+},{ }^{4} \mathrm{He}^{+}$, and ${ }^{16} \mathrm{O}^{+}$at incident energies $2,5,10,20,30,40$, and $53 \mathrm{keV}$. The TOF spectra were acquired using from HOPE-B, Pixel 3 measured during Calibration Phase 3 . We fit and normalize the data using an asymmetric Gaussian distribution (Stancik and Brauns 2008) to the mass spectra derived using (3):

$$
m_{\mathrm{F}}=A \exp \left(-\ln (2) \gamma^{2}\left(\frac{m-m_{0}}{m_{W}}\right)^{2}\right) .
$$

where $m_{0}$ is the location of the peak maximum and $m_{\mathrm{W}}$ is a width parameter of the mass peak. The asymmetry function is $\gamma=1+e^{a\left(m-m_{0}\right)}$, and the asymmetry parameter is $a$. The direction of skewness is reflected in the sign of $a$, and, when $a=0$, the peak is Gaussian and $m_{\mathrm{W}}$ corresponds to the full-width-at-half-maximum of the mass peak. The fit to this equation is shown in top panel Fig. 33 using values of $k=0.2,0.7$, and 2.6 for $\mathrm{H}^{+}, \mathrm{He}^{+}$, and $\mathrm{O}^{+}$, respectively, in Eq. (4). These mass spectra were obtained for monoenergetic ion beams directed into HOPE B, Pixel 3, and thus contain all effects that contribute to uncertainty in the mass spectrum except for the energy response of the ESA subsystem. The peaks are well-resolved over most of the energy range. The precise mass location of the maxima of the peaks varies slightly with energy, which is the result of inaccuracy of the asymmetric Gaussian fit as well as second order effects of energy loss not captured in the generic form of Eq. (4).

The only uncertainty not included in the mass spectra derived using a monoenergetic ion beam (top panel of Fig. 33) is the energy passband of the ESA subsystem. The first order Taylor Series expansion of Eq. (3) (McComas et al. 1990) yields mass uncertainty $\Delta m / m \sim \Delta E /\left(E+\left|e V_{\mathrm{TOF}}\right|\right)$ directly associated with the uncertainty in ion energy at the entrance surface of the foil, which is exclusively due to the ESA energy response. From this equation and noting that $\Delta E_{\mathrm{FWHM}} / E \approx 0.12$ for the HOPE ESA subsystem, it is clear that the width and shape of the ESA energy passband, and thus its impact on mass resolution, becomes small at low energies due to the acceleration of the ions by the bias applied to the Drift Box. The bottom panel of Fig. 33 shows the intrinsic HOPE mass resolution derived by convolving the (approximately) Gaussian energy response of the ESA subsystem described in Sect. 4.1.1 with the asymmetric Gaussian fits to the mass peaks derived for a monoenergetic ion beam (top panel). This bottom panel therefore includes all effects that contribute to the HOPE mass resolution. The mass peak corresponding to $\mathrm{O}^{+}$is distinct from $\mathrm{H}^{+}$and $\mathrm{He}^{+}$. The $\mathrm{H}^{+}$and $\mathrm{He}^{+}$peaks are well-separated except at the highest energies. When the $\mathrm{H}^{+}$ abundance is high relative to $\mathrm{He}^{+}$at these higher energies, the $\mathrm{He}^{+}$peak becomes a shoulder on the $\mathrm{H}^{+}$peak, and the analysis summarized in Fig. 33 forms the basis for determining the 


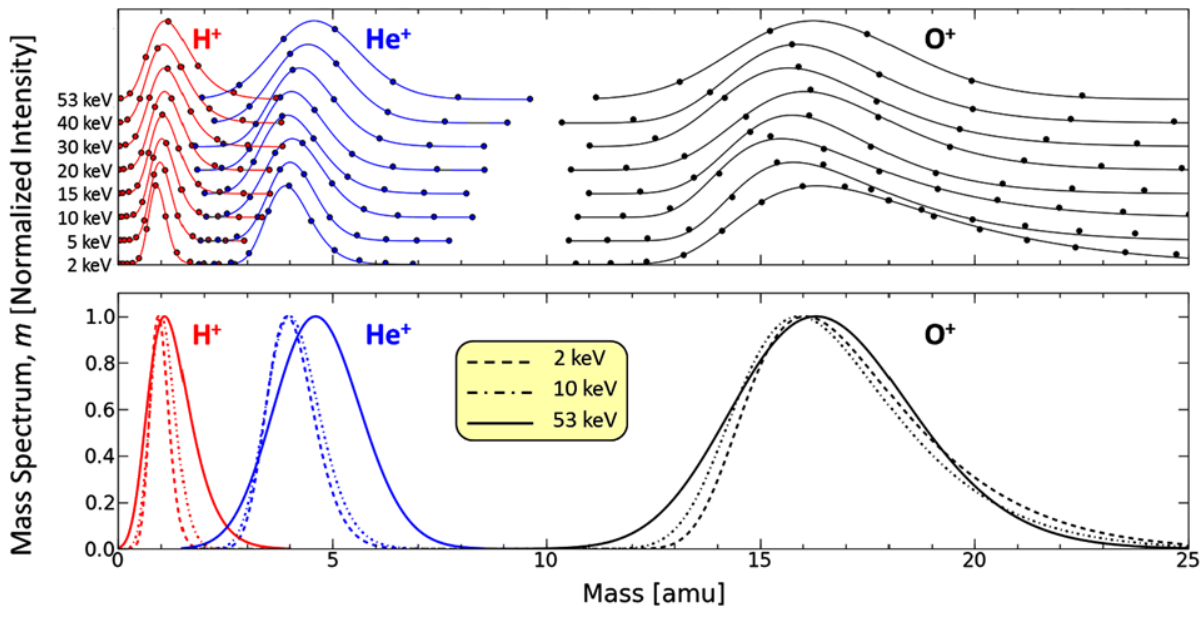

Fig. 33 (Top panel) Stacked TOF spectra derived for a monoenergetic beams of 2.0-53.5 $\mathrm{keV} \mathrm{H}^{+}($black), $\mathrm{He}^{+}$(blue), and $\mathrm{O}^{+}$(red) for HOPE B, Pixel 3. TOF spectra for other pixels for both HOPE-A and HOPE-B are similar. TOF data is converted to mass using Eq. (3). The solid lines are empirical fits to the data using an asymmetric Gaussian distribution (Eq. (5)). (Bottom panel) The convolution of asymmetric Gaussian fits of the mass spectra with the Gaussian energy response function of the ESA subsystem results in mass spectra $m$ that include all processes in HOPE that contribute to the instrument mass resolution

$\mathrm{He}^{+}$abundance. When compared to the top panel, the slight increase in widths of the $\mathrm{H}^{+}$ and $\mathrm{He}^{+}$mass peaks at $53 \mathrm{keV}$ in the bottom panel is due solely to due to the width of the energy passband.

The dominant effect governing the HOPE mass resolution is the cumulative time dispersion $\delta \tau_{\mathrm{TOF}}$ of the TOF subsystem and the timing electrons. While $\delta \tau_{\mathrm{TOF}}$ is generally independent of the incident particle mass and energy, a multitude of processes contribute to it, including scatter-induced pathlength differences of particles after exiting the foil, transport time variation of both Start and Stop electrons from their emission to their detection, timing jitter of each CEM detector, variation of propagation times of pulses between detectors of a single polar pixel, and timing variations within the timing electronics for each of the pixels. The TOF time dispersion becomes increasingly important for shorter TOF measurements (i.e., light ions at high energies), for which the time dispersion becomes a significant fraction of the uncertainty, contributing to the observed widening of the mass peaks of both $\mathrm{H}^{+}$ and $\mathrm{He}^{+}$at higher energies in the top panel of Fig. 33 .

The time dispersion $\delta \tau_{\mathrm{TOF}}$ of the TOF Subsystem and the timing electronics can be derived from the TOF spectra acquired in electron mode. The electron TOF across the $3 \mathrm{~cm}$ TOF drift region is short $(0.92 \mathrm{~ns}$ at $1.5 \mathrm{keV}$ and 0.61 at $52 \mathrm{keV})$ compared to $\delta \tau_{\mathrm{TOF}}$ and has minimal variation from energy lost by transit through the foil. Thus, incident electrons provide a nearly simultaneous stimulation of Start and Stop electrons, and variations in the TOF spectra produced by these electrons reflects the cumulative time dispersion of the entire subsystem. Figure 34 shows a TOF spectrum acquired in electron mode from all HOPE A pixels during commissioning. At each energy, the counts in the TOF spectrum are normalized to a maximum value of one for easier comparison across energies. As expected, the electron peak is clearly observed at $\tau_{\mathrm{TOF}}>10 \mathrm{~ns}$, which corresponds to the $10 \mathrm{~ns}$ time delay of the delay line in the Stop timing electronics that was incorporated to account for $\delta \tau_{\mathrm{TOF}}$ and to ensure a Start pulse is registered before a correlated Stop pulse. 

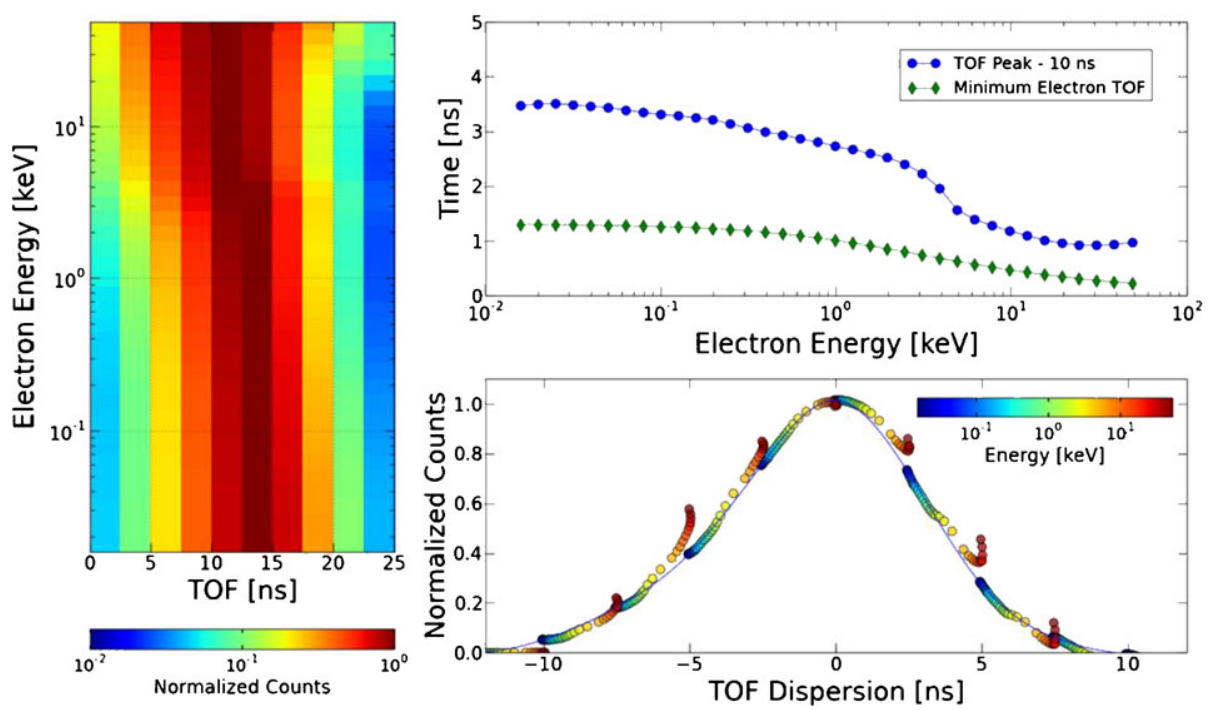

Fig. 34 HOPE TOF performance is demonstrated using TOF spectra in electron mode normalized to unity at each energy (left panel) acquired during HOPE commissioning. The location of the TOF peak (blue curve) is energy dependent (top right panel), and correcting for this time shift yields overlapping TOF spectra (bottom right panel) that reflect the fundamental time dispersion $\delta \tau_{\mathrm{TOF}}$ of the TOF subsystem

At each electron energy, we fit a Gaussian peak to the TOF peaks to find the time $\tau_{\text {MAX }}(E)$ corresponding to the maximum counts. The blue curve of Fig. 34, upper right panel, shows $\tau_{\text {MAX }}(E)-10 \mathrm{~ns}$, which is a factor of $\sim 2.5$ above the calculated electron TOF at its incident energy across the $3 \mathrm{~cm}$ of the Drift box (green curve). The difference between the measured TOF of the peak maximum and the calculated location can be attributed to an additional $\sim 1$ ns systematically observed over all pixels at the highest electron energies and, at lower energies, energy loss of the incident electron in the foil. After shifting in time the spectrum at each energy by $\tau_{\text {MAX }}(E)$, the TOF spectra at each energy overlap in time as shown in Fig. 34, bottom right panel. The peaks at higher incident electron energy show a slightly wider peak than at lower energies; this likely arises because the higher energy peaks have fewer total counts, and the peak is influenced by the underlying penetrating background which has a similar TOF spectrum and should be uniform in magnitude across all energies. We also note that these TOF peaks include the range in TOF from the different electron energies that are accepted within an individual energy passband; this is a small effect but is largest at the highest energies because the $1.5 \mathrm{keV}$ TOF box bias in electron mode efficiently accelerates low energy ions to nearly the same energy. We fit a single curve to the composite TOF peaks for all incident electron energies less than $11.5 \mathrm{keV}$ using a cubic spline interpolation and obtain the following full widths that characterize $\delta \tau_{\mathrm{TOF}}: 7.9 \mathrm{~ns}$ at half-maximum, $11.8 \mathrm{~ns}$ at the $25 \%$ level, and $10 \%=15.7 \mathrm{~ns}$ at the $10 \%$ level. This is the primary metric for the mass resolution of the HOPE mass spectrometer.

HOPE commissioning also provided the first complete ion TOF spectra, which are more closely representative of the HOPE performance in space than calibration data because the source ion population is broadly distributed in energy and over incident polar and azimuthal angles. Figure 35 shows an ion TOF spectrum from all five pixels of $\mathrm{HOPE} \mathrm{B} . \mathrm{H}^{+}, \mathrm{He}^{+}$, and $\mathrm{O}^{+}$are clearly observed. For these spectra, in which the $\mathrm{He}^{+}$abundance is low compared to the $\mathrm{H}^{+}$abundance, the $\mathrm{He}^{+}$peak is distinct from the $\mathrm{H}^{+}$peak for energies $\leq 20 \mathrm{keV}$; at 


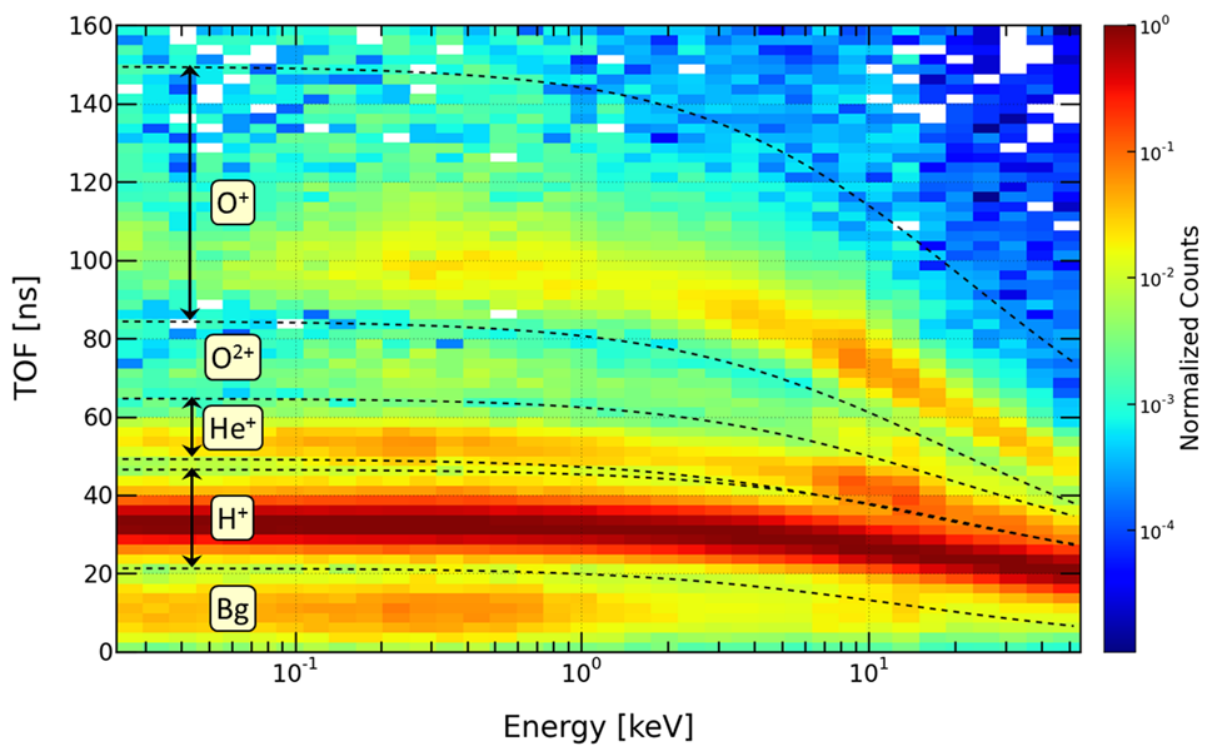

Fig. 35 HOPE A ion TOF spectra acquired over all five pixels. Counts at each energy passband have been normalized to the maximum counts observed for $\mathrm{H}^{+}$. The upper and lower TOF boundaries for $\mathrm{H}^{+}, \mathrm{He}^{+}$, and $\mathrm{O}^{+}$that are used for defining the TOF LUT are shown as black dashed lines. $\mathrm{O}^{2+}$ is also clearly observed but is not reported by HOPE as a distinct species. The background (labeled as $\mathrm{Bg}$ ) from penetrating radiation has a short TOF spectrum similar to electrons

higher energies, the $\mathrm{He}^{+}$peak becomes a shoulder of the $\mathrm{H}^{+}$peak and thus requires a more sophisticated abundance algorithm, based on calibration results, to quantify the relative $\mathrm{H}^{+}$ and $\mathrm{He}^{+}$abundances. $\mathrm{O}^{2+}$ is also clearly observed, but its abundance is comparatively small and is not tracked as a species identified in situ by HOPE. Because of the speed at which penetrating radiation traverses the TOF drift region, its TOF is characteristically short and similar to electron TOF spectra. The short TOF peak characteristic of penetrating radiation is also clearly observed in the spectra.

Composition information is obtained in situ by binning the data on board the spacecraft by TOF value in three time windows. A unique timing window with minimum and maximum byte values is defined for each of the three ion species $\mathrm{H}^{+}, \mathrm{He}^{+}$, and $\mathrm{O}^{+}$. The binning is performed by an onboard look up table (LUT), which allows for different binning for each polar pixel and each energy step. We use the $\mathrm{H}^{+}, \mathrm{He}^{+}$, and $\mathrm{O}^{+}$TOF peaks in Fig. 35 to derive empirical functions that are used to define the minimum and maximum TOF boundaries of the HOPE LUT. These are listed in Table 6 and shown as the dashed lines in Fig. 35 for nominal HOPE operation with $V_{\mathrm{TOF}}=-11.0 \mathrm{kV}$. These time values are converted to TOF byte values for incorporation into the LUT.

HOPE routinely provides full TOF spectra such as in Fig. 35 for two polar pixels per spacecraft spin, accumulated over all energies for a single azimuthal sector. TOF spectra are acquired for the other polar pixels over subsequent spins, enabling long-term monitoring of the TOF measurement integrity, monitoring penetrating background at short times-of-flight, and for analysis of, for example, $\mathrm{O}^{2+}$ that is not reported as a unique ion species. The TOF byte values in the LUT are identical for both HOPE A and B and have been updated based on analysis of the HOPE commissioning data. These LUTS can be modified in the future 
Table 6 Ion species time-of-flight definitions for both HOPE A and B time-of-flight LUTs

\begin{tabular}{|c|c|c|c|c|}
\hline \multicolumn{5}{|c|}{$\tau_{\mathrm{TOF}}[\mathrm{ns}]=a_{1}+\frac{a_{2}}{\sqrt{a_{3} E[\mathrm{keV}] \pm V_{\mathrm{TOF}}[\mathrm{kV}]}}$} \\
\hline Species & Boundary & $a_{1}$ & $a_{2}$ & $a_{3}$ \\
\hline \multirow[t]{2}{*}{$\mathrm{H}^{+}$} & Min & -1.02 & 74.2 & 1.630 \\
\hline & $\operatorname{Max}$ & 11.78 & 115.4 & 0.861 \\
\hline \multirow[t]{2}{*}{$\mathrm{He}^{+}$} & Min & 15.65 & 111.5 & 1.480 \\
\hline & $\operatorname{Max}$ & 14.73 & 166.0 & 1.120 \\
\hline \multirow[t]{2}{*}{$\mathrm{O}^{+}$} & Min & 8.18 & 253.0 & 1.180 \\
\hline & $\operatorname{Max}$ & 18.18 & 435.6 & 0.969 \\
\hline
\end{tabular}

to accommodate any changes needed based on evolving scientific objectives or changes in HOPE operational parameters.

\subsubsection{Backgrounds and Noise}

The response to UV light was measured using a $5 \mathrm{~W}, 195 \mathrm{~nm}$ mercury lamp. Both HOPE-A and HOPE-B showed fewer than $2.5 \times 10^{-13}$ singles counts per photon, and no coincidence counts were observed. These count rates meet the HOPE requirements, which assume a geocoronal Ly-a flux of $10^{9}$ photons $/ \mathrm{cm}^{-2} \mathrm{~s}^{-1} \mathrm{sr}^{-1}$.

The intrinsic HOPE detector noise during calibration was quantified by examining CEM counts in the absence of particle input. With no incident ions or electrons, each pixel showed 10-100 Hz of singles (Starts or Stops) noise counts. This represents $<1$ count for each individual 10.4 ms energy step accumulation interval. Start noise counts were generally observed to be slightly higher than Stop noise counts, typically by factors of no more than $2-3$. Coincidence counts are even lower, typically $<2-30 \mathrm{~Hz}$. These noise rates were monitored throughout instrument testing and calibration for both HOPE-A and HOPE-B. Some temporal variation as well as variation between polar pixels was observed.

The HOPE response to an energetic particle background was determined by measurements in the presence of a radioactive source. This test measured the signature of penetrating radiation in HOPE in electron and ion modes. During calibration, a $15 \mathrm{mCi}^{60} \mathrm{Co}$ source was placed at the exterior surface of a 1.27-cm-thick vacuum chamber glass window, $11.2 \mathrm{~cm}$ from HOPE-A. The 1.17 and $1.33 \mathrm{MeV} \gamma$-rays are a reasonable proxy for penetrating radiation because they generate photoelectrons and subsequent secondary electron showers deep within the instrument. HOPE-A was operated with the source absent and present in a neutral state in which $V_{\mathrm{TOF}}=0 \mathrm{kV}$, in electron mode with $V_{\mathrm{TOF}}=+1.5 \mathrm{kV}$, and ion mode with $V_{\mathrm{TOF}}=-11 \mathrm{kV}$.

Figure 36 shows coincident count rates (left panel) and singles count rates (right panel) for these three operational configurations. Measurements when the source was present are indicated by shaded regions; measurements when the source was removed are shown with a white background. The presence of the source clearly has a significant effect on the instrument count rate, although some variation of background independent of source present is observed over the test interval. Count rates were consistent across all five polar pixels and highest in Pixel 3, as expected since it was closest to the source. The presence of the source resulted in additional Start and Stop counts at a rate of $200-500 \mathrm{~Hz}$, with the Start and Stop count rates similar for electron mode and Stops consistently higher than Starts for ion mode. The coincidence rates, typically $<5-50 \mathrm{~Hz}$ for all pixels, were significantly higher in electron mode than ion mode. 

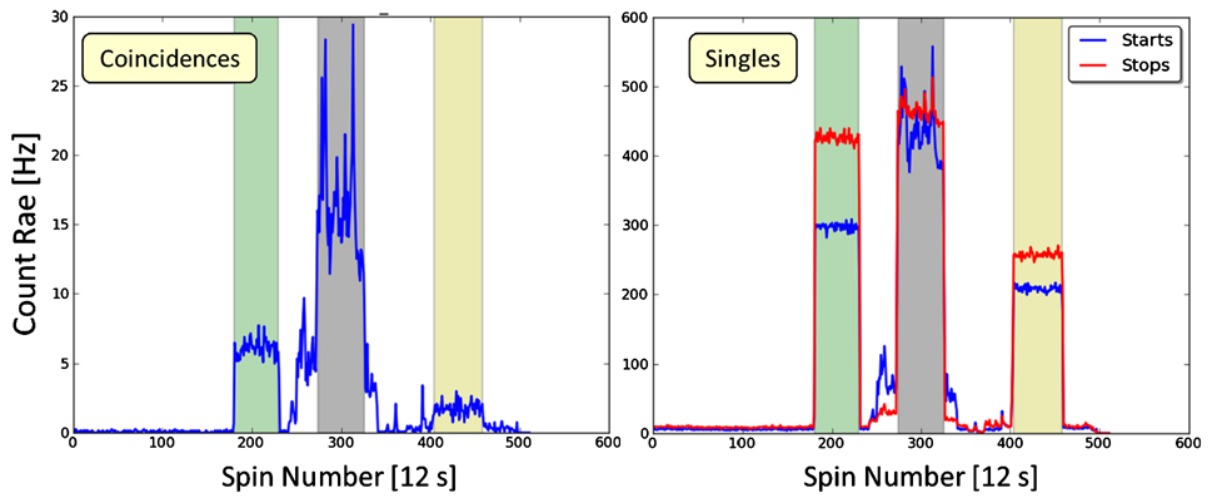

Fig. 36 Coincident (left panel) and singles (right panel) count rates for HOPE-A Pixel 2 during exposure to ${ }^{2}{ }^{60} \mathrm{Co} \gamma$-ray source, which serves as a proxy for penetrating radiation. Neutral state $\left(V_{\mathrm{TOF}}=0 \mathrm{kV}\right)$ measurements are shown in green, electron mode with $V_{\mathrm{TOF}}=+1.5 \mathrm{kV}$ in gray, and ion mode with $V_{\mathrm{TOF}}=-11 \mathrm{kV}$ in yellow. Times with white background are with no source present

Geant 4 modeling of HOPE of this test geometry and ${ }^{60}$ Co source strength (including attenuation in the vacuum window) predicted singles Start and Stop count rates of $\sim 400 \mathrm{~Hz}$, similar to the observed counts. As described previously in the Penetrating Radiation Analysis section, the same Geant 4 modeling of HOPE in the radiation belts predicted a background coincidence rate due to penetrating radiation at $<1 \mathrm{~Hz}$. This combination of measurements and analysis thus confirms that HOPE is sufficiently insensitive to the expected energetic particle background of the radiation belts.

\subsection{HOPE Observation Strategy}

HOPE has several operational modes that we now describe.

\subsubsection{HVSCI: Normal Science Mode}

Because of the complexity of synchronizing HOPE stepping supplies with a spacecraftprovided spin pulse, HOPE uses its own clock to cycle voltages and to toggle between ion and electron mode. Data are collected on a fixed time cadence that depends on the duration of an energy step $\tau_{\mathrm{ESA}}$ STEP, with the $\sim 12$ sec electron and ion frame acquisition time designed to be close to the nominal spacecraft spin period. This scheme simplifies the high voltage operations since each plasma measurement always has a fixed duration. Another consequence of this timing cadence is that the HOPE telemetry rate is solely based on the time interval of a HOPE energy step, regardless of spacecraft spin rate. We note that $\tau_{\text {ESA_STEP }}$ is adjustable over a limited range, and in flight we set this value such that a HOPE data frame is comparable to the actual spacecraft spin (spin rate predicted to be between 4-6 RPM). The following discussion of data projects assumes a nominal $12 \mathrm{sec}$ spacecraft spin period.

The HOPE mass spectrometer measures ions and electrons during alternate spins, and a HOPE Master Frame in nominal science mode is therefore composed of a pair of consecutive ion and electron data frames. A Master Frame is acquired over $\sim 24 \mathrm{sec}$.

Each data frame is divided into 16 azimuthal sectors, with data collected over the entire 72-step energy range for each sector (Fig. 37), giving a full energy spectrogram 16 times per data frame. However, because of telemetry limitations, in nominal HVSCI mode data from adjacent energy steps are combined on-board, and data are transmitted for the resulting 36 


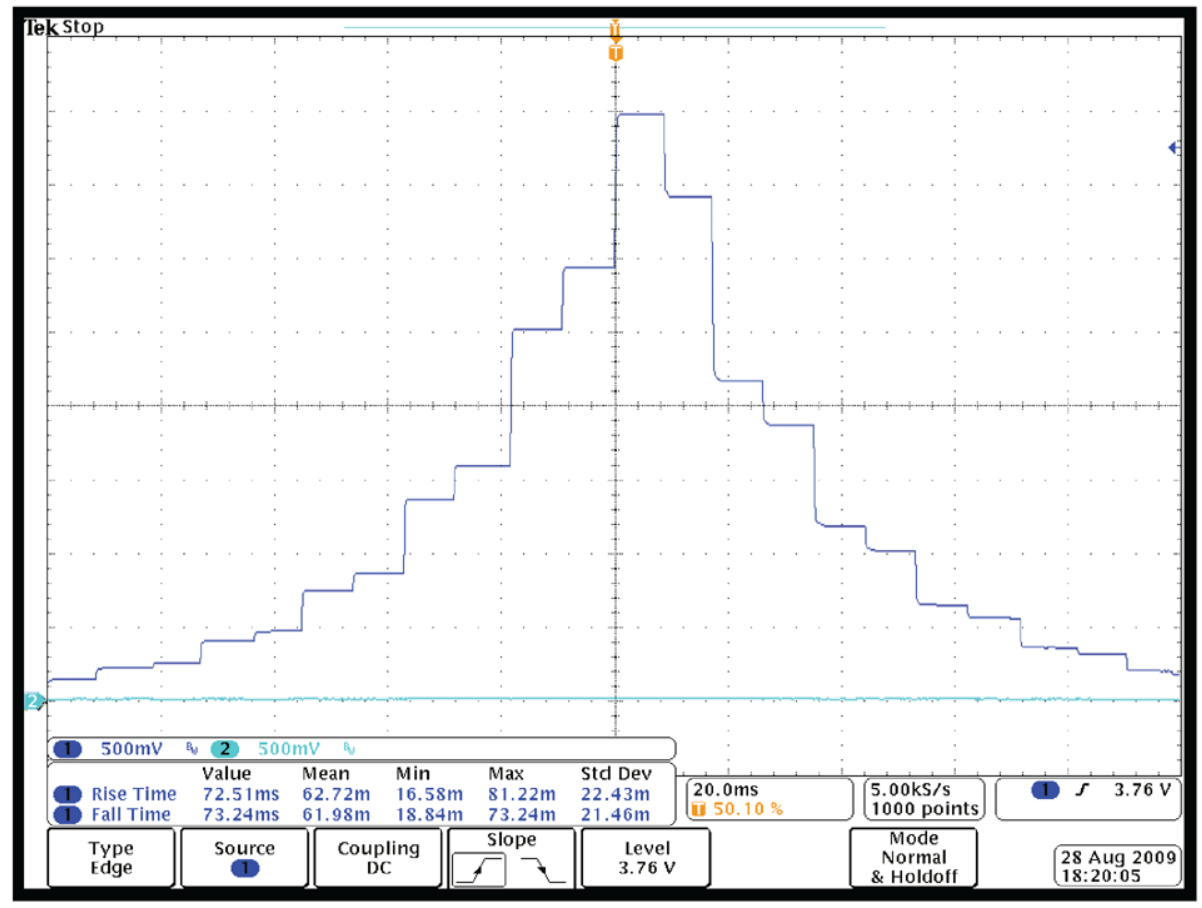

Fig. 37 This snapshot of the ESA voltage sweep in nominal HVSCI mode shows pairing of adjacent energy steps and interleaving of these pairs as the voltage is swept up and down. The time cadence of HOPE data acquisition is based on the time of a single energy step $\tau_{\text {ESA_STEP }}$

energy steps. Within each sector, voltages are stepped from $0 \mathrm{~V}$ to the maximum value (either negative or positive) and back down in an interleaved windshield-wiper mode, to ensure that the power supply is at $0 \mathrm{~V}$ when the polarity is changed. Of the 36 transmitted steps, odd-numbered steps are collected on the way up to maximum $V_{\mathrm{ESA}}$ and even-numbered steps on the way down as shown in Fig. 37. The 72 energies are thus measured in the order $1-2,5-6,9-10, \ldots, 69-70,72-71,68-67, \ldots, 8-7,4-3$.

As mentioned above, data from adjacent azimuthal sectors for some pixels are also combined, again for telemetry reasons, with a scheme that provides nearly equivalent solid angle measurements for each pixel as illustrated in Fig. 38. For the $\pm 72^{\circ}$ polar pixels, data from the 16 azimuthal sectors are combined in groups of 4 , resulting in 4 azimuthal sectors per HOPE data frame. For the $\pm 36^{\circ}$ polar pixels, data are combined in groups of 2, resulting in 8 azimuthal sectors per HOPE data frame. Data from the $0^{\circ}$ polar pixel retains the complete 16 azimuthal measurements.

The HOPE data collection was designed to be highly flexible to accommodate different measurement strategies that might evolve based on scientific discoveries throughout the mission. A single on-board LUT, which can be changed as necessary, includes voltage levels for each step, combinations of voltage steps for a full scan, and combinations of scans for a larger data collection plan. The LUT also includes energy and azimuthal summing information for each data collection mode. Table 7 shows the primary science data returned by HOPE, whose total telemetry allocation is $9.32 \mathrm{kbps}$.

For each polar pixel and azimuthal sector, both valid coincident and non-coincident (singles) counts are reported. A valid coincidence count corresponds to a detected particle whose 

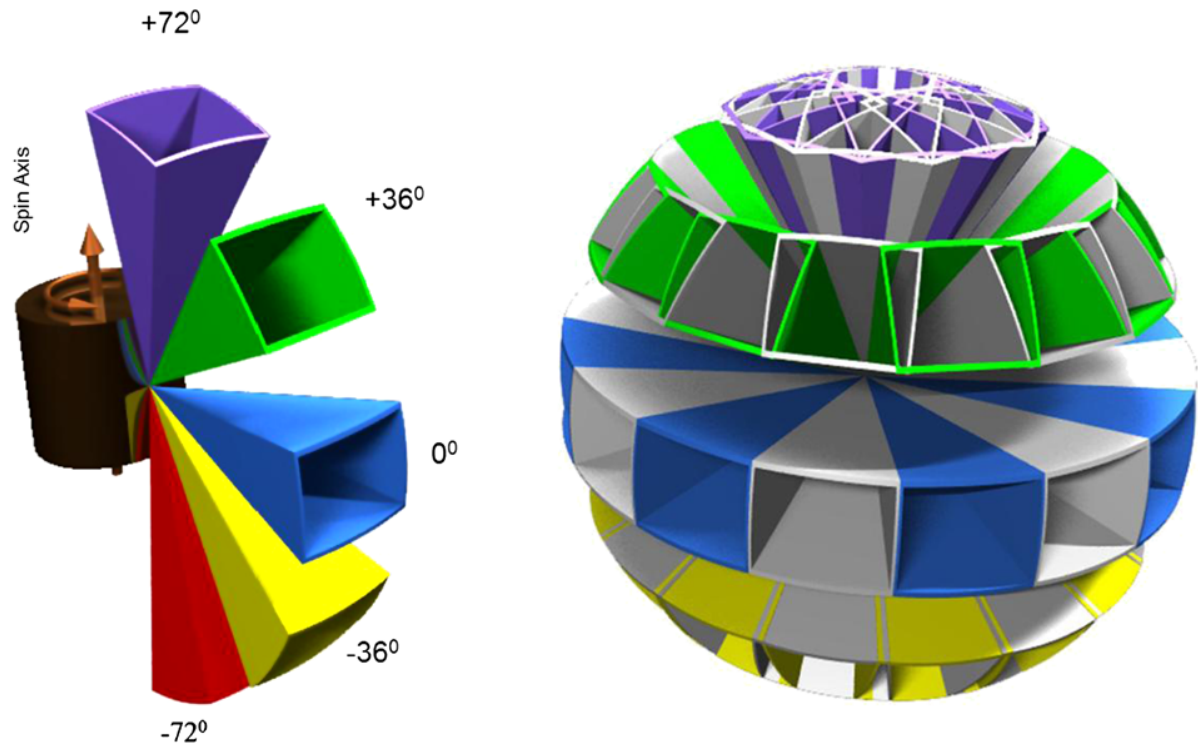

Fig. 38 The HOPE polar pixels (left panel) are centered at polar angles $0^{\circ}, \pm 36^{\circ}$, and $\pm 72^{\circ}$, where the $0^{\circ}$ polar pixel views perpendicular to the spacecraft spin axis. Over a complete spin, measurements over 16 azimuthal sectors are accumulated. At higher polar angles, these azimuthal sectors overlap as shown in the right panel

Table 7 Primary HOPE science data

\begin{tabular}{llllllll}
\hline Data frame & $\begin{array}{l}\text { Energy } \\
\text { bins }\end{array}$ & $\begin{array}{l}\text { Data } \\
\text { products }\end{array}$ & $\begin{array}{l}\text { Polar } \\
\text { pixels }\end{array}$ & $\begin{array}{l}\text { Average } \\
\text { spin/azim } \\
\text { sectors }\end{array}$ & $\begin{array}{l}\text { Bits/ } \\
\text { sample }\end{array}$ & $\begin{array}{l}\text { No. per } \\
\text { master } \\
\text { frame }\end{array}$ & $\begin{array}{l}\text { Data rate } \\
{[\mathrm{kbps}]}\end{array}$ \\
\hline $\begin{array}{l}\text { Electron data } \\
\text { frame }\end{array}$ & 36 & $4^{\mathrm{a}}$ & 5 & $8^{\mathrm{c}}$ & 8 & 1 & 1.92 \\
$\begin{array}{l}\text { Ion data } \\
\text { frame }\end{array}$ & 36 & $5^{\mathrm{b}}$ & 5 & $8^{\mathrm{c}}$ & 8 & 1 & 2.40 \\
$\begin{array}{l}\text { Full TOF } \\
\text { matrix }\end{array}$ & 36 & 64 & $2^{\mathrm{e}}$ & $1^{\mathrm{d}}$ & 8 & 2 & 3.07 \\
\hline
\end{tabular}

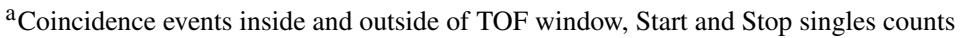

${ }^{\mathrm{b}}$ Coincidence events for $\mathrm{H}^{+}, \mathrm{He}^{+}, \mathrm{O}^{+}$; total Start single counts; total Stop singles counts

${ }^{\mathrm{c}}$ Data is acquired for all 16 azimuthal sectors for each polar pixel; azimuthal pixels are then combined from 16-16-16-16-16 to 4-8-16-8-4 for polar pixels located at $-72^{\circ},-36^{\circ}, 0^{\circ},+36^{\circ}$ and $+72^{\circ}$, respectively

${ }^{\mathrm{d}}$ Option to select and cycle through specific pixels or azimuthal sectors

${ }^{\mathrm{e}} 1$ polar pixel (= one angular sector) per 2 spins; cycles through all CEMs for ions and electrons every 10 spins

${ }^{\mathrm{f}}$ Master Frame $=24 \mathrm{sec}$ assuming $12 \mathrm{sec}$ spin period. Complete ion and electron histograms are acquired during alternate spins

TOF lies within a defined TOF window for $\mathrm{H}^{+}, \mathrm{He}^{+}$, and $\mathrm{O}^{+}$in ion mode. In electron mode, valid electron counts correspond to coincident events within the short TOF window that is equivalent to the $\mathrm{H}^{+}$TOF bin in ion mode. Also in electron mode, coincidence counts within 
a longer TOF window that is far beyond any realistic electron TOF are also reported as a measure of background coincidence rate, likely from penetrating radiation.

Total start and stop counts are also transmitted, and subtraction of valid coincident counts from the start and stop counts yields the singles (non-coincident) counts. Inconsistency of the singles counts relative to the expected valid coincidence counts based on derived composition provides yet another method to monitor background rates. Also, during times of minimal penetrating radiation, the singles and valid coincidence rates are the basis for calculation of the absolute efficiency of each of the detector pixels.

Full TOF spectra (64 TOF bins with 2.5-ns bin width) are transmitted at all 36 energies for a single spin sector per spin for 2 polar pixels. Over time, these spectra step through the polar pixels and over azimuthal sectors $0,4,8$, and 12 (of the 16 possible sectors), providing TOF information over the full HOPE measurement space every 10 Master Frames. TOF spectra are collapsed in energy and azimuthal angle in the same way as the corresponding counters data. The exact number of reported full TOF spectra can be adjusted by the HEU to fill the available telemetry. All data undergo $15 \rightarrow 8$ bit compression to maximize use of the telemetry allocation. HOPE also routinely transmits housekeeping information, including instrument temperatures, high and low voltage levels, CEM current monitors, and CEM threshold levels.

\subsubsection{Burst Support Mode}

In addition to the routine data collection described above, HOPE can also collect data in a burst support mode. This mode is designed to enhance wave-particle interaction science by supporting the burst modes of the RBSP wave instruments, EMFISIS and EFW. HOPE burst support mode uses the same data packets and total data rate as nominal science operations, with onboard burst support LUTs used to select electron or ion mode, energy, and angle selection. Burst support mode can be initiated in two ways. In "synoptic" mode burst support data are collected at regular, pre-determined time intervals throughout the orbit. For initial operations, we plan to collect one burst support Master Frame every 633 s (once every 26 Master Frames).

In "triggered" mode, HOPE collects burst support data by responding to requests sent by the EMFISIS or EFW instruments in the shared spacecraft data packet. Requests from the waves instruments are limited to no more than 2 spins out of 60 sequential spins for each of the two instruments, to ensure that sufficient data are collected in normal science mode.

Up to seven unique burst support tables can be stored onboard HOPE, allowing flexibility in the data collection. In addition, these LUTs can be updated in flight if another scheme is deemed more useful. For example, for initial burst support operations, HOPE will operate using a LUT that measures only electrons at 9 energy levels over nearly the full HOPE energy range with high time resolution $(0.1875 \mathrm{~s}$ per measurement) and with no azimuthal compression for any of the polar pixels. These measurements focus on rapid acquisition of a distribution function with meaningful information on the temperature anisotropy, which requires fitting a pitch angle distribution capable of distinguishing near parallel and near perpendicular energy spectra.

\subsubsection{Space Weather Data Support}

HOPE will provide data to the real time space weather telemetry stream, consisting of ion and electron counts at 4 energies. Nominal energies for these measurements are $0.25,0.3,10$, and $40 \mathrm{keV}$. Measurements from Pixels 1, 2, 4, and 5 will be collapsed over all azimuthal 
angles. Measurements from Pixel 3 will be provided in eight $45^{\circ}$ azimuthal bins. Space weather data are generated at all times, but are based on energy steps from the nominal energy table. Thus at times when a different table is being used (i.e. burst support mode), these real time data will not represent the expected energies. Although the HOPE space weather data represent only a small fraction of the full HOPE data set, they provide a quicklook overview of particle distributions and their variability in real time. These data will be made available for real-time space weather modeling and forecasting efforts.

\subsubsection{Data Processing Levels}

The data continuously generated by the HOPE instrument will be stored on the satellite until daily contact with a ground station. The data will be then downlinked and stored under the control of the Mission Operation Center (MOC), which will transfer the data to the ECT Science Operation Center (SOC) at the Los Alamos National Laboratory. These data will be in the form of raw telemetry data, containing both science and housekeeping data used to monitor and maintain HOPE. The ECT SOC is responsible for the processing and disseminating the HOPE scientific data and data products, which includes applying the instrument response function and, with the HOPE team, validating the data. Higher-level products combine the data of all ECT instruments.

During normal mission operations, the MOC collects all raw telemetry during contact times and combines these data into a daily MET (Mission Elapsed Time) Level zero (L0) files. The ECT SOC obtains these L0 files as soon as they are available at the MOC, on a roughly daily cadence. The first processing step is to produce Level one (L1) data, daily files in UTC, that contain science data in count rates and state of health $(\mathrm{SOH})$ data in engineering units on standard time tags.

Level two (L2) data is the first useable science data product and consists of instrument counts of L1 data converted to ion and electron flux as a function of energy, angle, and species by applying the HOPE instrument response function. Also included are estimates of backgrounds from penetrating radiation derived from HOPE. Validation of HOPE data occurs primarily using L2 data.

L3 and L4 data products incorporate data from other RBSP instruments such as local magnetic field direction, enabling higher level data products such as pitch angle distributions, moments, and L-shell association. L4 data products generate phase space distributions and incorporate regional and global models, such as magnetic field models. L3 and L4 use a common set of utilities and codes, since all ECT instrument data in L2 format have a high degree of commonality (count rates per detector, spin sector and energy).

\section{Summary}

The HOPE mass spectrometer has been designed to measure plasma electron and ion fluxes from $1 \mathrm{eV}-50 \mathrm{keV}$ and to distinguish ion composition of the three dominant species $\mathrm{H}^{+}$, $\mathrm{He}^{+}$, and $\mathrm{O}^{+}$within the harsh environment of the Earth's radiation belts. The HOPE measurement methodology and instrument design have been driven to reduce the potentially enormous background from penetrating radiation in the radiation belts. Measurements made by HOPE will thus enable discovery of the physical processes that drive the dynamics and evolution of the radiation belts. 
Acknowledgements The HOPE mass spectrometer is the result of an outstanding collaboration between scientists, engineers, technicians, and support personnel at multiple institutions. In particular, we gratefully appreciate the seamless collaboration with the other instrument teams of the ECT instrument suite (at Aerospace Corporation and University of Colorado) as well as engagement, expertise, and specialty engineering provided by the broader RBSP team, primarily at APL/JHU. Work at Los Alamos National Laboratory was performed under the auspices of the United States Department of Energy under Interagency Purchase Request NNG07EK09I.

Open Access This article is distributed under the terms of the Creative Commons Attribution License which permits any use, distribution, and reproduction in any medium, provided the original author(s) and the source are credited.

\section{Appendix: LANL Space Plasma Instrument Calibration Facility}

Los Alamos National Laboratory operates the Space Plasma Instrument Calibration Facility for test and calibration of space physics instrumentation, detector development and characterization, ultrathin foil characterization, and charged particle optics testing and characterization. The Facility consists of an ion accelerator, a Class 10,000 clean room, a nearby Class 1000 clean room, and a supporting lab in which a new electron cyclotron resonance (ECR) ion source, capable of generating ions and charge states characteristic of the solar wind, will be operational. All vacuum systems use hydrocarbon-free vacuum pumps (cryopumps or oil-free turbopumps) to prevent hydrocarbon poisoning of electron multiplier detectors such as microchannel plates (MCPs) and channel electron multipliers (CEMs). The facility has tested and calibrated numerous subsystems and instruments on NASA missions, including SWEPAM on ACE; SWOOPS on Ulysses; IMS and IBS on Cassini; GEM, GIM, and the Solar Wind Concentrator on Genesis; MENA on IMAGE; the TWINS neutral atom imagers; the IBEX-Hi neutral atom imager on IBEX; and the HOPE mass spectrometers on RBSP.

The ion accelerator provides a monoenergetic beam of atomic or molecular ions over a broad energy range $0.4-60 \mathrm{keV} / q$ where $q$ is the ion charge. It's microwave ion source (Leung et al. 1985; Walther et al. 1986) generates primarily $q=+1$ ions and a smaller fraction of $q=+2$ ions from both solid and gaseous sources. Advantages of this source over a duoplasmatron ion source include its high stability (beam current varies $<5 \%$ per hr), ease of use, low intrinsic energy spread of $<2 \mathrm{eV}$ (Chamberlin et al. 1987), ability to generate (and not fail during) oxygen beam operation (Walther et al. 1987), and extremely low maintenance requirements.

The ion beam is accelerated and focused using an electrostatic Einzel lens embedded in the accelerator column. The beam then enters a water-cooled electromagnet located $137 \mathrm{~cm}$ from the exit of the microwave cavity of the ion source. The electro-magnet has 5 exit ports located at bend angles of $0^{\circ}, \pm 30^{\circ}$, and $\pm 60^{\circ}$ relative to the incident ion beam; two of these ports are dedicated beamlines, instrument calibration chamber $\left(+60^{\circ}\right.$, entrance is $242 \mathrm{~cm}$ from the exit of the electromagnet) and an experimental chamber $\left(-60^{\circ}\right.$, entrance is $172 \mathrm{~cm}$ from the exit of the electromagnet) for characterizing and testing smaller components and research devoted to development of new detection technologies. Adjustable apertures in the form of vertical and horizontal slits are located $46 \mathrm{~cm}$ upstream of the magnet entrance and $82 \mathrm{~cm}$ downstream of the magnet exit, in accordance with Barber's rule, i.e., the upstream and downstream transverse-beam focal points and the locus of the bend radius of the sector magnet all lie along the same line (Barber 1933). Quadrupole electrostatic deflection plates (two for vertical deflection and two for horizontal deflection) have inner radii $1.0 \mathrm{~cm}$ and length $17.8 \mathrm{~cm}$ and are located $100 \mathrm{~cm}$ after the exit of the electromagnet and $\sim 165 \mathrm{~cm}$ upstream of the aperture of an instrument in the calibration chamber. A DC voltage applied 


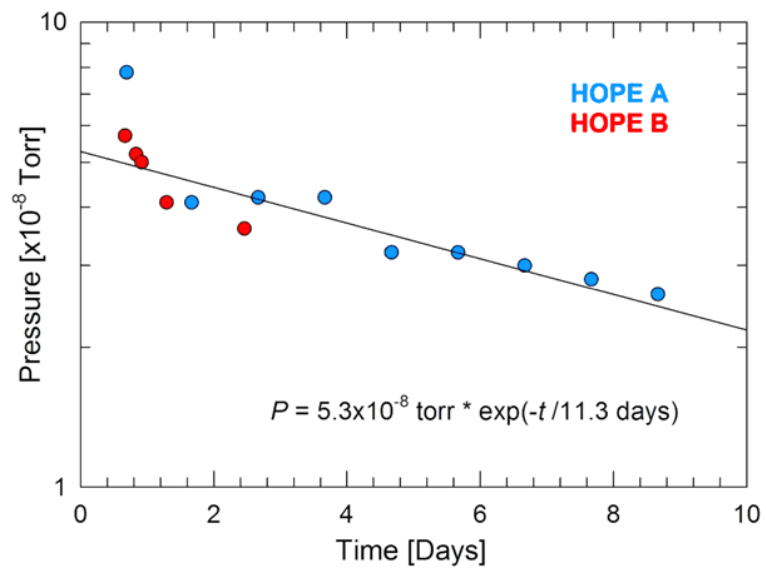

Fig. 39 The calibration chamber pressure is shown as a function of pumpdown time for the HOPE A and B flight units for Phase 3 calibration (after detector refurbishment and flight HVPS installation). After initial pumpdown, the pressure decreased with a characteristic time constant of 11.3 days

to these plates steers the beam, and a periodic bipolar triangular waveform voltage applied on top of this DC level (at slightly different vertical and horizontal frequencies) enables raster scanning of the beam over a broad area. The measured mass resolution of the accelerator is $m / \Delta m>200$.

The intrinsic angular divergence of a typical ion beam is $\leq 0.07^{\circ}$ at the instrument calibration chamber, as measured using an imaging microchannel plate detector located in the calibration chamber. The maximum divergence of the beam at the edge of a $2 \mathrm{~cm}$ wide aperture at the entrance of an instrument is $0.35^{\circ}$.

An electron source (Henderson et al. 2012) based on UV photoelectron emission from an aluminum photocathode was developed and used for HOPE electron testing and calibration. UV photons with sufficient energy to generate photoelectrons from an Al 6061 plate were generated using a light emitting diode (LED) (Thorlabs Model LED260W) and accelerated through a 12-in-dia aperture. The maximum electron energy attained was $20 \mathrm{keV}$. Helmholtz coils were installed to cancel the Earth's magnetic field and prevent deflection of the electron beam, enabling calibration at electron energies as low as $0.6 \mathrm{keV}$.

The calibration chamber is 1.4 - $\mathrm{m}$ diameter $\times 1.0$-m wide $(\sim 1500$ liters $)$. Three cryopumps are used for high pumping speed and for cleanliness, especially to minimize hydrocarbon contamination, to which CEM and MCP detectors are particularly susceptible. The chamber pressure was typically at $5 \times 10^{-8}$ Torr with a flight instrument installed, and the pumpdown rates for the HOPE A and B flight mass spectrometers are shown in Fig. 39. The chamber has an instrument platform with computer-controlled polar, azimuthal, and linear (perpendicular to beam axis) motions. Vibration, shock, thermal, and EMI/EMC testing facilities in the same building complex were used for HOPE.

\section{References}

S. Agostinelli et al., Geant4-a simulation toolkit. Nucl. Instrum. Methods Phys. Res., Sect. A, Accel. Spectrom. Detect. Assoc. Equip. 506(3), 250-303 (2003). doi:10.1016/S0168-9002(03)01368-8

F. Allegrini, D.J. McComas, D.T. Young, J.-J. Berthelier, J. Covinhes, J.-M. Illiano, J.-F. Riou, H.O. Funsten, R.W. Harper, Energy loss of 1-50 keV H, He, C, N, O, Ne, Ar ions transmitted through thin carbon foils. Rev. Sci. Instrum. 77, 044501 (2006). doi:10.1063/1.2185490 
P.C. Anderson, W.B. Hanson, W.R. Coley, W.R. Hoegy, Spacecraft potential effects on the Dynamics Explorer 2 satellite. J. Geophys. Res. 99(A3), 3985-3997 (1994). doi:10.1029/93JA02104

D.N. Baker, J.B. Blake, R.W. Klebesadel, P.R. Higbie, Highly relativistic electrons in the earth's outer magnetosphere. I. Lifetimes and temporal history 1979-1984. J. Geophys. Res. 91(A4), 4265-4276 (1986). doi:10.1029/JA091iA04p04265

S.J. Bame, D.J. McComas, M.F. Thomsen, B.L. Barraclough, R.C. Elphic, J.P. Glore, J.T. Gosling, J.C. Chavez, E.P. Evans, F.J. Wymer, Magnetospheric plasma analyzer for spacecraft with constrained resources. Rev. Sci. Instrum. 64, 1026 (1993). doi:10.1063/1.1144173

R.A. Baragiola, E.V. Alonso, A. Oliva Florio, Electron emission from clean metal surfaces induced by low energy light ions. Phys. Rev. B 19, 121-129 (1979). doi:10.1103/PhysRevB.19.121

N.F. Barber, Note on the shape of an electron beam bent in a magnetic field. Proc. Leeds Philos. Lit Soc., Sci. Sect. 2, 427-434 (1933)

L.W. Blum, E.A. MacDonald, S.P. Gary, M.F. Thomsen, H.E. Spence, Ion observations from geosynchronous orbit as a proxy for ion cyclotron wave growth during storm times. J. Geophys. Res. 114, A10214 (2009). doi:10.1029/2009JA014396

L.W. Blum, E.A. MacDonald, L.B.N. Clausen, X. Li, A comparison of magnetic field measurements and a plasma-based proxy to infer EMIC wave distributions at geosynchronous orbit. J. Geophys. Res. 117, A05220 (2012). doi:10.1029/2011JA017474

J. Bortnik, R.M. Thorne, N.P. Meredith, Modeling the propagation characteristics of chorus using CRRES suprathermal electron fluxes. J. Geophys. Res. 112, A08204 (2007). doi:10.1029/2006JA012237.112

C.W. Carlson, D.W. Curtis, G. Paschmann, W. Michael, An instrument for rapidly measuring plasma distribution functions with high resolution. Adv. Space Res. 2, 67-70 (1983). doi:10.1016/02731177(82)90151-X

D.L. Carpenter, R.R. Anderson, An ISEE/Whistler model of equatorial electron density in the magnetosphere. J. Geophys. Res. 97(A2), 1097-1108 (1992). doi:10.1029/91ja01548

D.L. Carpenter, Earth's plasmasphere awaits rediscovery. Eos 76(9), 89 (1995). doi:10.1029/ 95EO00041

C. Cattell et al., Discovery of very large amplitude whistler-mode waves in earth's radiation belts. Geophys. Res. Lett. 35, L01105 (2008). doi:10.1029/2007GL032009

E.P. Chamberlin, K.N. Leung, S. Walthers, R.A. Bibeau, R.L. Stice, G.M. Kelley, J. Wilson, Measurement of the beam energy spread from a microwave ion source. Nucl. Instrum. Methods Phys. Res., Sect. B, Beam Interact. Mater. Atoms 26, 227-234 (1987). doi:10.1016/0168-583X(87)90755-5

Y. Chen, G.D. Reeves, R. Friedel, The energization of relativistic electrons in the outer Van Allen radiation belt. Nat. Phys. 3, 614 (2007). doi:10.1038/Nphys655

D.A. Dahl, SIMION for the personal computer in reflection. Int. J. Mass Spectrom. 200(1-3), 3-25 (2000). doi:10.1016/S1387-3806(00)00305-5

I. Daglis, R. Thorne, W. Baumjohann, S. Orsini, The terrestrial ring current: origin, formation, and decay. Rev. Geophys. 37(4), 407-438 (1999). doi:10.1029/1999RG900009

H. Demers, N. Poirier-Demers, A.R. Couture, D. Joly, M. Guilmain, N. De Jonge, D. Drouin, Threedimensional electron microscopy simulation with the CASINO Monte Carlo software. Scanning 33, 135-146 (2011). doi:10.1002/sca.20262

A.J. Dessler, E.N. Parker, Hydromagnetic theory of geomagnetic storms. J. Geophys. Res. 64, 12 (1959). doi:10.1029/JZ064i012p02239

L.A. Dietz, J.C. Sheffield, Secondary electron emission induced by 5-30-keV monatomic ions striking thin oxide films. J. Appl. Phys. 46, 4361-4370 (1975). doi:10.1063/1.321463

D. Drouin, A.R. Couture, D. Joly, X. Tastet, V. Aimez, R. Gauvin, CASINO V2.42 - a fast and easy-to-use modeling tool for scanning electron microscopy and microanalysis users. Scanning 29, 92-101 (2007). doi:10.1002/sca. 20000

H.O. Funsten, D.J. McComas, B.L. Barraclough, Thickness uniformity and pinhole density analysis of thin carbon foils using keV ions. Nucl. Instrum. Methods Phys. Res., Sect. B, Beam Interact. Mater. Atoms 66, 470-478 (1992a). doi:10.1016/0168-583X(92)95421-M

H.O. Funsten, B.L. Barraclough, D.J. McComas, Pinhole detection in thin foils used in space plasma diagnostic instrumentation. Rev. Sci. Instrum. 63, 4741-4743 (1992b). doi:10.1063/1.1143626

H.O. Funsten, D.J. McComas, B.L. Barraclough, Ultrathin foils used for low energy neutral atom imaging of the terrestrial magnetosphere. Opt. Eng. 32, 3090-3095 (1993). doi:10.1117/12.149187

H.O. Funsten, M. Shappirio, Sputtering of thin carbon foils by $20 \mathrm{keV}$ and $40 \mathrm{keV} \mathrm{Ar}{ }^{+}$bombardment. Nucl. Instrum. Methods Phys. Res., Sect. B, Beam Interact. Mater. Atoms 127, 905-909 (1997). doi:10.1016/S0168-583X(97)00079-7

H.O. Funsten, R.W. Harper, D.J. McComas, Absolute detection efficiency of space-based ion mass spectrometers and neutral atom imagers. Rev. Sci. Instrum. 76, 053301 (2005). doi:10.1063/1.1889465 
H.O. Funsten, A.A. Guthrie, R.W. Harper, K.H. Kihara, M.P. Manzo, M.J. Fagan, D.J. McComas, S. Weidner, F. Allegrini, D. Everett, B. Rodriguez, G. Dunn, J. Hanley, M. Maple, K. Mashburn, S. Pope, P. Valek, E. Moebius, J. Nolin, S. Ellis, D. Heirtzler, B. King, H. Kucharek, S. Turco, S. Zaffke, D. Reisenfeld, P. Janzen, S.A. Fuselier, M. Gruntman, E. Roelof, P. Wurz, D. Piazza, L. Saul, P. Bochsler, The interstellar boundary explorer high energy (IBEX-Hi) neutral atom imager. Space Sci. Rev. 146, 75-103 (2009). doi:10.1007/s11214-009-9504-y

S.P. Gary, Theory of Space Plasma Microinstabilities (Cambridge Univ. Press, New York, 1993)

S.A. Glauert, R.B. Horne, Calculation of pitch angle and energy diffusion coefficients with the PADIE code. J. Geophys. Res. 110, A042046 (2005). doi:10.1029/2004JA010851

J.T. Gosling, J.R. Asbridge, S.J. Bame, W.C. Feldman, Effects of a long entrance aperture upon the azimuthal response of spherical section electrostatic analyzers. Rev. Sci. Instrum. 49, 1260 (1978). doi:10.1063/1.1135566

J.C. Green, M.G. Kivelson, Relativistic electrons in the outer radiation belt: differentiating between acceleration mechanisms. J. Geophys. Res. 109, A03213 (2004). doi:10.1029/2003JA010153

K.C. Henderson, R.W. Harper, H.O. Funsten, E. MacDonald, Ultraviolet stimulated electron source for use with low energy plasma instrument calibration. Rev. Sci. Instrum. 83, 073308 (2012). doi:10.1063/1.4732810

R.B. Horne, R.M. Thorne, Potential waves for relativistic electron scattering and stochastic acceleration during magnetic storms. Geophys. Res. Lett. 25(15), 3011 (1998). doi:10.1029/98GL01002

R.B. Horne, G.V. Wheeler, H.St.C.K. Alleyne, Proton and electron heating by radially propagating fast magnetosonic waves. J. Geophys. Res. 105(A12), 27,597-27,610 (2000). doi:10.1029/2000JA000018

J.C. Ingraham, T.E. Cayton, R.D. Belian, R.A. Christensen, R.H.W. Friedel, M.M. Meier, G.D. Reeves, M. Tuszewski, Substorm injection of relativistic electrons to geosynchronous orbit during the great magnetic storm of March 24, 1991. J. Geophys. Res. 106(A11), 25759-25776 (2001). doi:10.1029/2000ja000458

V.K. Jordanova, J.U. Kozyra, G.V. Khazanov, A.F. Nagy, C.E. Rasmussen, M.-C. Fok, A bounce-averaged kinetic-model of the ring current ion population. Geophys. Res. Lett. 21(25), 2785-2788 (1994). doi:10.1029/94GL02695

H.J. Kim, A.A. Chan, Fully-adiabatic changes in storm-time relativistic electron fluxes. J. Geophys. Res. 102(A10), 22107-22116 (1997). doi:10.1029/97JA01814

S.M. Krimigis, G. Gloeckler, R.W. McEntire, T.A. Potemra, F.L. Scarf, E.G. Shelley, Magnetic storm of September 4, 1984: a synthesis of ring current spectra and energy densities measured with AMPTE/CCE. Geophys. Res. Lett. 12(5), 329-332 (1985). doi:10.1029/GL012i005p00329

K.N. Leung, S.R. Walther, H.W. Owren, A compact microwave ion source. IEEE Trans. Nucl. Sci. NS-32, 1803-1805 (1985). doi:10.1109/TNS.1985.4333729

X. Li, I. Roth, M. Temerin, J.R. Wygant, M.K. Hudson, J.B. Blake, Simulation of the prompt energization and transport of radiation belt particles during the March 24, 1991 SSC. Geophys. Res. Lett. 20(22), 2423-2426 (1993). doi:10.1029/93GL02701

X. Li, D.N. Baker, S.G. Kanekal, M. Looper, M. Temerin, Long term measurements of radiation belts by SAMPEX and their variations. Geophys. Res. Lett. 28(20), 3827-3830 (2001). doi:10.1029/2001GL013586

J. Lindhard, M. Scharff, H.E. Schiøtt, Range concepts and heavy ion ranges (Notes on atomic collisions II). Mat. Fys. Medd. K. Dan. Vidensk. Selsk. 33, 14 (1963)

D. Lowenstein, A. Rusek, Technical developments at the NASA space radiation laboratory. Radiat. Environ. Biophys. 46(2), 91-94 (2007). doi:10.1007/s00411-006-0084-x

L.R. Lyons, R.M. Thorne, Equilibrium structure of radiation belt electrons. J. Geophys. Res. 78(13), 21422149 (1973). doi:10.1029/JA078i013p02142

E.A. MacDonald, M.F. Thomsen, H.O. Funsten, Background in channel electron multiplier detectors due to penetrating radiation in space. IEEE Trans. Nucl. Sci. 53(3), 1593-1598 (2006). doi:10.1109/TNS.2006.874497

E.A. MacDonald, M.H. Denton, M.F. Thomsen, S.P. Gary, Superposed epoch analysis of a whistler instability criterion at geosynchronous orbit during geomagnetic storms. J. Atmos. Sol.-Terr. Phys. 70(14), 17891796 (2008). doi:10.1016/j.jastp.2008.03.021

E.A. MacDonald, H.O. Funsten, E.E. Dors, M.F. Thomsen, P. Janzen, R.M. Skoug, G.D. Reeves, J.T. Steinberg, R. Harper, D. Young, J.-M. Jahn, D. Reisenfeld, New magnetospheric ion composition measurement techniques, in Future Perspectives of Space Plasma and Particle Instrumentation and International Collaborations: Proceedings of the International Conference. AIP Conf. Proc., vol. 1144, (2009), pp. 168-172

E.A. MacDonald, L.W. Blum, S.P. Gary, M.F. Thomsen, M.H. Denton, High speed stream driven inferences of global wave distributions at geosynchronous orbit; relevance to radiation belt dynamics. Proc. R. Soc. A 466(2123), 3351-3362 (2010). doi:10.1098/rspa.2010.0076 
D.J. McComas, J.E. Nordholt, S.J. Bame, B.L. Barraclough, J.T. Gosling, Linear electric field mass analysis: a technique for three-dimensional high mass resolution space plasma composition measurements. Proc. Natl. Acad. Sci. USA 87(15), 5925-5929 (1990). doi:10.1073/pnas.87.15.5925

D.J. McComas, F. Allegrini, C.J. Pollock, H.O. Funsten, S. Ritzau, G. Gloeckler, Ultra-thin ( $\sim 10 \mathrm{~nm})$ carbon foils in space instrumentation. Rev. Sci. Instrum. 75(11), 4863-4870 (2004). doi:10.1063/1.1809265

N.P. Meredith, R.M. Thorne, R.B. Horne, D. Summers, B.J. Fraser, R.R. Anderson, Statistical analysis of relativistic electron energies for cyclotron resonance with EMIC waves observed on CRRES. J. Geophys. Res. 108(A6), 1250 (2003). doi:10.1029/2002JA009700

M. Nosé, A. Ieda, S.P. Christon, Geotail observations of plasma sheet ion composition over 16 years: on variations of average plasma ion mass and $\mathrm{O}^{+}$triggering substorm model. J. Geophys. Res. 114, A07223 (2009). doi:10.1029/2009JA014203

T. Obara, T. Nagatsuma, M. Den, Y. Miyoshi, A. Morioka, Main-phase creation of 'seed' electrons in the outer radiation belt. Earth Planets Space 52(1), 41-47 (2000)

G.S. Oehrlein, R.J. Phaneuf, D.B. Graves, Plasma-polymer interactions: a review of progress in understanding polymer resist mask durability during plasma etching for nanoscale fabrication. J. Vac. Sci. Technol. 29(1), 010801 (2011). doi:10.1116/1.3532949

T.P. O’Brien, M.B. Moldwin, Empirical plasmapause models from magnetic indices. Geophys. Res. Lett. 30(4), 1 (2003). doi:10.1029/2002GL016007

T.I. Pulkkinen, N.A. Tsyganenko, R.H.W. Friedel (eds.), The Inner Magnetosphere: Physics and Modeling (Am. Geophys. Union, Washington, 2005)

G.D. Reeves, Relativistic electrons and magnetic storms: 1992-1995. Geophys. Res. Lett. 25(11), 1817-1820 (1998). doi:10.1029/98GL01398

G.D. Reeves, K.L. McAdams, R.H.W. Friedel, T.P. O’Brien, Acceleration and loss of relativistic electrons during geomagnetic storms. Geophys. Res. Lett. 30(10), 1529-1532 (2003). doi:10.1029/2002g1016513

G.D. Reeves, Radiation belt storm probes: the next generation of space weather forecasting. Space Weather 5, 11 (2007). doi:10.1029/2007sw000341

G.D. Reeves, A.A. Chan, C. Rodger, New directions for radiation belt research. Space Weather 7, S07004 (2009). doi:10.1029/2008sw000436

G.D. Reeves, Y. Chen, G.S. Cunningham, R.W.H. Friedel, M.G. Henderson, V.K. Jordanova, J. Koller, S.K. Morley, M.F. Thomsen, S. Zaharia, Dynamic radiation environment assimilation model: DREAM. Space Weather 10, S03006 (2012). doi:10.1029/2011sw000729

S.M. Ritzau, R.A. Baragiola, Electron emission from carbon foils induced by keV ions. Phys. Rev. B 58(5), 2529-2538 (1998). doi:10.1103/PhysRevB.58.2529

J.G. Roederer, On the adiabatic motion of energetic particles in a model magnetosphere. J. Geophys. Res. 72(3), 981-992 (1967). doi:10.1029/JZ072i003p00981

G. Santina, P. Nieminen, H. Evansa, E. Daly, F. Lei, P. Truscott, C. Dyer, B. Quaghebeur, D. Heynderickx, New Geant 4 based simulation tools for space radiation shielding and effects analysis. Nucl. Phys. B, Proc. Suppl. 125, 69-74 (2003). doi:10.1016/S0920-5632(03)90968-6

D.M. Sawyer, J.I. Vette, AP-8 trapped proton environment for solar maximum and solar minimum. NASA STI/Recon Tech. Rep. 77, 18983 (1976)

M. Schulz, L.J. Lanzerotti, Particle Diffusion in the Radiation Belts (Springer, New York, 1974)

N. Sckopke, A general relation between the energy of trapped particles and the disturbance field near the earth. J. Geophys. Res. 71(13), 3125-3130 (1966). doi:10.1029/JZ071i013p03125

H. Seiler, Secondary electron emission in the scanning electron microscope. J. Appl. Phys. 54(11), R1-R18 (1983). doi:10.1063/1.332840

R.S. Selesnick, J.B. Blake, Dynamics of the outer radiation belt. Geophys. Res. Lett. 24(11), 1347-1350 (1997). doi:10.1029/97GL51409

R.S. Selesnick, M.D. Looper, R.A. Mewaldt, A theoretical model of the inner proton radiation belt. Space Weather 5, S04003 (2007). doi:10.1029/2006SW000275

M.L. Spasojevic, L.W. Blum, E.A. MacDonald, S.A. Fuselier, D.I. Golden, Correspondence between a plasma-based EMIC wave proxy and subauroral proton precipitation. Geophys. Res. Lett. 38, L23102 (2011). doi:10.1029/2011GL049735

A.L. Stancik, E.B. Brauns, A simple asymmetric lineshape for fitting infrared absorption spectra. Vib. Spectrosc. 47, 66-69 (2008). doi:10.1016/j.vibspec.2008.02.009

D. Summers, R.M. Thorne, F. Xiao, Relativistic theory of wave-particle resonant diffusion with application to electron acceleration in the magnetosphere. J. Geophys. Res. 103(A9), 20487-20500 (1998). doi:10.1029/98JA01740

M. Taylor, R.H.W. Friedel, G.D. Reeves, M.W. Dunlop, T.A. Fritz, P.W. Daly, A. Balogh, Multisatellite measurements of electron phase space density gradients in the earth's inner and outer magnetosphere. J. Geophys. Res. 109, A05220 (2004). doi:10.1029/2003ja010294 
S. Väyrynen, J. Räisänen, P. Tikkanen, I. Kassamakov, E. Tuominen, Effects of activation by proton irradiation on silicon particle detector electric characteristics. J. Appl. Phys. 106(2), 024908 (2009). doi:10. $1063 / 1.3168436$

M.F. Thomsen, M.H. Denton, V.K. Jordanova, L. Chen, R.M. Thorne, Free energy to drive equatorial magnetosonic wave instability at geosynchronous orbit. J. Geophys. Res. 116, A08220 (2011). doi:10.1029/2011JA016644

D.L. Turner, Y. Shprits, M. Hartinger, V. Angelopoulos, Explaining sudden losses of outer radiation belt electrons during geomagnetic storms. Nat. Phys. 8, 208-212 (2012). doi:10.1038/NPHYS2185

C.G.H. Walker, M.M. El-Gomati, A.M.D. Assa'd, M. Zadrazil, The secondary electron emission yield for 24 solid elements excited by primary electrons in the range $250-5000 \mathrm{eV}$ : a theory/experiment comparison. Scanning 30(5), 365-380 (2008). doi:10.1002/sca.20124

S.R. Walther, K.N. Leong, W.B. Kunkel, Characteristics of a compact microwave ion source. Rev. Sci. Instrum. 57, 1531-1535 (1986). doi:10.1063/1.1139197

S.R. Walther, K.N. Leong, K.W. Ehlers, W.B. Kunkel, Generation of oxygen, carbon, and metallic ion beams by a compact microwave ion source. Nucl. Instrum. Methods Phys. Res., Sect. B, Beam Interact. Mater. Atoms 21, 215-217 (1987). doi:10.1016/0168-583X(87)90830-5

M. Wüest, Time-of-flight ion composition measurement technique for space plasmas, in Measurement Techniques in Space Plasmas: Particles, ed. by F. Pfaff, E. Borovsky, T. Young. Geophys. Monogr. Ser., vol. 102 (AGU, Washington, 1998), pp. 141-155. doi:10.1029/GM102p0141

D.T. Young, S.J. Bame, M.F. Thomsen, R.H. Martin, J.L. Burch, J.A. Marshall, B. Reinhard, 2-Pi-radian field-of-view toroidal electrostatic analyzer. Rev. Sci. Instrum. 59(5), 743-751 (1988). doi:10.1063/ 1.1139821 